\title{
EVOLVING SPIKING NEURAL NETWORKS FOR PATTERN CLASSIFICATION PROBLEMS
}

\section{SHIRIN DORA}

School of Computer Science and Engineering

\author{
A thesis submitted to the Nanyang Technological \\ University in partial fulfilment of the requirements for the degree \\ of Doctor of Philosophy
}





\section{Acknowledgement}

First and foremost, I would like to thank my advisor Dr. Suresh Sundaram for his continuous support, guidance and encouragement throughout my PhD. I am grateful to him for patiently dealing with my queries and mistakes. His mentoring during my $\mathrm{PhD}$ tenure has helped me grow both as a person and as a researcher.

I would also like to thank Dr. Narasimhan Sundararajan for providing insightful comments and suggestions. He enthusiastically shared his experiences in our frequent discussions which have helped me improve my understanding of the subject.

I will always stay obliged to NTU and Computational Intelligence Laboratory for offering me an opportunity and platform to pursue my higher education.

I am also thankful to all my friends and colleagues at NTU who made my academic journey pleasant and memorable.

I am grateful to my parents and my brother for happily standing by me while I pursued my PhD. I am thankful to my wife for having faith in me and motivating me throughout this period. Without their unconditional love, I would not have been able to complete my thesis work. 


\section{Contents}

Abstract vii

List of Figures $\quad$ xiv

List of Tables $\quad$ xvi

1 Introduction 1

1.1 Motivation and Challenges . . . . . . . . . . . . . 1

1.2 Objectives of this Study . . . . . . . . . . . . . . 5

1.3 Major Contributions . . . . . . . . . . . . . . 7

1.4 Organization of the Thesis . . . . . . . . . . . . . . . . 11

2 A Review of Literature on Spiking Neural Networks 15

2.1 Neuron Models . . . . . . . . . . . . . . . . . . . . . 17

2.1.1 Leaky Integrate-and-Fire Model . . . . . . . . . . . . . . . . . . 17

2.1.2 Spike Response Model . . . . . . . . . . . . . . . . . . . . . . . 18

2.1.3 Izhikevich Neuron Model . . . . . . . . . . . . . . . . . . . . . 20

2.2 Learning Algorithms for Spiking Neural Networks . . . . . . . . . . . . 21

2.2.1 Gradient based Learning Algorithms . . . . . . . . . . . . 21

2.2.2 Spike Timing Dependent Plasticity Based Learning Algorithms . . 23

2.2.3 Rank Order Based Learning Algorithms . . . . . . . . . . . 26

2.2.4 Spike Convolution Based Learning Algorithms . . . . . . . . . . . 27

2.3 Applications . . . . . . . . . . . . . . . . . . . . . . . 28

2.3.1 Face detection and identification . . . . . . . . . . . . . . . 29

2.3.2 NeuCube Applications . . . . . . . . . . . . . . . . . . . . . . . 29 
2.3.3 Applications in Energy Sector . . . . . . . . . . . . . . 30

2.4 Summary . . . . . . . . . . . . . . . . . . . . . 30

3 A Self-Regulating Evolving Spiking Neural Classifier 33

3.1 Self-Regulating Evolving Spiking Neural Classifier . . . . . . . . . . . . . 34

3.1.1 Population Coding . . . . . . . . . . . . . . . . . . . . 35

3.1.2 Architecture of the SRESN Classifier . . . . . . . . . . . . . 36

3.1.3 Learning Algorithm for the SRESN Classifier . . . . . . . . . . . 38

3.2 Working of the SRESN Classifier Using the Ionosphere Problem . . . . . 48

3.3 Performance Comparison of the SRESN Classifier . . . . . . . . . . 53

3.3.1 Iris Flower Classification Problem . . . . . . . . . . . . . . . 55

3.3.2 Wisconsin Breast Cancer Problem . . . . . . . . . . . . . . . . . 57

3.4 Discussion on the Limitations of the SRESN classifier . . . . . . . . . 58

3.5 Summary . . . . . . . . . . . . . . . . . . . . 59

4 A Two Stage Margin Maximization Spiking Neural Network $\quad 61$

4.1 Two Stage Margin Maximizing Spiking Neural Network for Classification Problems . . . . . . . . . . . . . . . . . . . . . 62

4.1.1 Architecure of TMM-SNN . . . . . . . . . . . . . . 63

4.1.2 Learning Algorithm for TMM-SNN . . . . . . . . . . . . 66

4.2 Working of TMM-SNN Using the Ionosphere Problem . . . . . . . . . . 75

4.3 Performance Evaluation of the TMM-SNN . . . . . . . . . . . . 78

4.3.1 Performance Comparison with Batch Learning Algorithms . . . . 80

4.3.2 Performance Comparison with Evolving Spiking Neural Network . 85

4.3.3 Performance Comparison with Accurate Synaptic-Efficiency Adjustment ......................... 87

4.4 Summary . . . . . . . . . . . . . . . . . . . 89

5 An Online Meta-neuron based Learning Algorithm $\quad 91$

5.1 Spiking Neural Network with a Meta-neuron . . . . . . . . . . . . . 93

5.2 Online Meta-neuron Based Learning Algorithm for Pattern Classification Problems . . . . . . . . . . . . . . . . . . . . . . . . 99 
5.3 Working of OMLA Using the Ionosphere Problem . . . . . . . . . . 105

5.4 Performance Evaluation of the Online Meta-neuron Based Learning Algorithm . . . . . . . . . . . . . . . . . . . . 109

5.5 Summary . . . . . . . . . . . . . . . . . . . . . . 113

6 Neuromorphic Implementation of the Online Meta-neuron based Learn$\begin{array}{ll}\text { ing Algorithm } & 117\end{array}$

6.1 Motivation . . . . . . . . . . . . . . . . . . 118

6.2 Neuromorphic Implementation of the Online Meta-neuron Based Learning Algorithm . . . . . . . . . . . . . . . . . . . . . . . . . 119

6.3 Performance Evaluation _ . . . . . . . . . . . . . . . . . . . . . . 129

6.3.1 Study on Performance of OMLA with Reduced Bit Precision using Ionosphere Problem . . . . . . . . . . . . . . . . . . . . 130

6.3.2 Comparison of OMLA-RBP and Software Simulations of OMLA . 132

6.4 Summary . . . . . . . . . . . . . . . . . . . . . . . . 134

7 Conclusions and Future Works 135

7.1 Conclusions . . . . . . . . . . . . . . . . . . . . 135

$7.2 \quad$ Future Directions . . . . . . . . . . . . . . . . . . . . . 138

7.2.1 Encoding Real Valued Features Into Spike Trains . . . . . . . . . 138

7.2.2 Improvements in the Meta-neuron Based Learning Rule . . . . . . 138

7.2.3 Address Event Representation (AER) Based Communication Protocol .............................. 139

7.2.4 Extension to Regression Problems . . . . . . . . . . . . . . . . . 140

$\begin{array}{ll}\text { Author's Publications } & 141\end{array}$

$\begin{array}{ll}\text { Bibliography } & 143\end{array}$ 


\section{Abstract}

This thesis focuses on the development of new batch/online learning algorithms for evolving spiking neural networks that can be used for pattern classification problems. The input and output signals of spiking neurons consist of discrete events (spikes) in time. The inherent discontinuous nature of spikes is an issue in developing learning algorithms for spiking neurons. This has inspired many researchers to study plasticity mechanisms observed in the brain to develop efficient learning techniques for spiking neurons. Spike Timing Dependent Plasticity (STDP) is one of the most studied biological plasticity mechanism. STDP relies only on the locally available information to update the weights of a given synapse. The local nature of STDP may result in an unbalanced distribution of weights and lead to convergence issues.

Similar to the previous generations of neural networks, selecting an appropriate spiking neural network architecture for approximating the relationship between a set of input and output spike patterns is a challenging problem. To address this issue, rank order learning based evolving Spiking Neural Network (eSNN) has been proposed. Rank order learning takes into account the global information, but it ignores the precise time of spikes. Further, eSNN uses a two layered network to approximate the decision boundary, which may require a higher number of neurons to approximate the relationship between the input and output spike patterns. This thesis is directed towards three main problems in the development of learning algorithms for spiking neural networks, viz. utilizing both local and global information, evolving the network architecture and learning in an online framework, specifically for pattern classification problems.

The first contribution of this work is the development of a Self-Regulating Evolving Spiking Neural (SRESN) classifier with a two layered network. The SRESN classifier operates in a batch learning framework and uses heuristic learning strategies to evolve the network architecture and simultaneously update the synaptic weights. Depending on the information present in a sample with respect to the knowledge stored in the network, it chooses to either add a neuron or update the network parameters or skip learning a sample. The SRESN classifier uses rank order learning in a feature-wise manner for initializing the weights of a newly added neuron and for updating the weights of existing neurons. This helps the SRESN classifier in achieving better generalization performance 
and faster convergence.

The second contribution of this work is a Two-stage Margin Maximization Spiking Neural Network (TMM-SNN) that employs a three layered SNN. The learning algorithm of TMM-SNN has two stages, namely, structure learning stage and output weights learning stage. In the first stage (structure learning stage), the learning algorithm evolves and updates weights of the hidden neurons. In this stage, the learning algorithm evolves the hidden layer completely in the first epoch and updates the weights of hidden neurons using margin maximization based update rule for multiple epochs. In the first epoch, a new neuron is added such that the newly added neuron spikes at a specific time. For this purpose, an activation based coding scheme is developed which uses the locally available information to initialize the weights of a new neuron. At the end of the first stage, the learning algorithm fixes the synaptic weights and thresholds of hidden neurons. In the second stage (output weights learning stage), the learning algorithm updates the output neuron weights, such that the temporal separation between the spike times of the interclass and intraclass neurons is maximized. The performance of TMM-SNN is statistically compared with the other existing learning algorithms for SNNs on benchmark problems. The performance results of different algorithms are compared using training/testing accuracy, number of epochs and number of network parameters. The results of the performance evaluation clearly indicate that TMM-SNN can achieve better performance using fewer epochs.

The local update strategies in TMM-SNN do not take into account the global information stored in the network while updating the weights of a given synapse. As a result, TMM-SNN requires multiple presentations of the training spike patterns to closely approximate the relationship between input spike patterns and the corresponding class labels.

The third contribution of this work is the development of a new concept of metaneuron for a two layered SNN for learning in a single presentation (online learning) of the input spike patterns. The concept of meta-neuron is inspired by the role of astrocytes in modulating synaptic plasticity in the brain. Astrocytes can connect to multiple synapses simultaneously, intercept the activities on the connected synapses and modulate the plasticity of these synapses. This form of heterosynaptic plasticity allows consideration of both global information stored in the network along with the local information present in the input spike patterns to update the weights of a given synapse.

The meta-neuron can intercept the activities of the presynaptic neurons and can access the weights of existing synapses in the network. This allows the meta-neuron to utilize the locally available information in the activity of a presynaptic neuron and the globally available information in the form of synaptic weights. A meta-neuron based learning rule is developed that utilizes both the local and global information to produce 
precise shifts in the spike times of the postsynaptic neurons. The capability of the metaneuron based learning rule to produce precise shifts in the spike times of the postsynaptic neuron renders it suitable for use in an online learning framework. To demonstrate this capability, an Online Meta-neuron based Learning Algorithm (OMLA) is developed that evolves the network architecture and updates the synaptic weights of neurons in the network. The performance of OMLA is statistically compared with the other existing online as well as batch learning algorithms for spiking neural networks. Performance comparison results clearly indicate that OMLA performs better than other existing learning algorithms for spiking neural networks. To study the suitability of OMLA for real applications, this thesis also presents a possible neuromorphic implementation of OMLA using a Field Programmable Gate Array (FPGA). The purpose of this study is to examine the implementation of the newly developed OMLA from a feasibility perspective.

The digital implementation of OMLA employs spiking neurons modeled using the spike response function in the hardware. The spike response function is simulated in the hardware using the COordinate Rotation DIgital Computer (CORDIC) circuit. The performance of the neuromorphic implementation of OMLA has been evaluated on several benchmark data sets. The results of performance evaluation clearly indicate that the neuromorphic implementation closely emulates the software based simulations. A more rigorous study to develop a neuromorphic device optimized for hardware is a topic for future work. 
Chapter 0. Abstract 


\section{List of Figures}

1.1 Different phases of an action potential . . . . . . . . . . . 2

2.1 An RC circuit used to model a leaky integrate-and-fire neuron . . . . . . 18

2.2 Membrane potential of a neuron modeled using the spike response model

2.3 Examples of STDP causing (a) long term potentiation and (b) long term

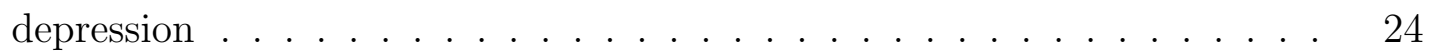

2.4 Learning window in STDP . . . . . . . . . . . . . . . . . . 24

2.5 An illustrative example of the rank order learning using five presynaptic neurons and a single postsynaptic neuron . . . . . . . . . . . . . . . 27

3.1 An illustrative example of the population coding using four receptive fields for a feature in the range $[0,1]$. The activation strength of different receptive fields is encoded according to different line styles. . . . . . . . . . . . 36

3.2 The SRESN classifier uses a two layered spiking neural network. The input layer consists of $m P$ neurons for a problem with $m$ features and $P$ being the number of receptive fields used to encode each feature into a spike pattern. Each input neuron generates a single spike in the simulation interval and the time of spike generated by the $f^{\text {th }}$ neuron encoding the $i^{\text {th }}$ feature is denoted by $t_{i f} \ldots \ldots \ldots \ldots \ldots \ldots \ldots$. . . . . . . . . . . . . 37

3.3 An illustrative example of the 'skip sample strategy' . . . . . . . . . . 39

3.4 Illustrative examples of adding a neuron to handle novelty . . . . . . . . 41 
3.5 An illustrative example of the procedure for initializing weights and threshold of a newly added neuron using a problem with two features, each encoded using five receptive fields. (a) Initial value of weights, determined using modified rank order learning, for the input neurons encoding the first feature, (b) PSP of the newly added neuron in the interval $[0, T]$ with initialized weights. The PSP of the neuron at $T_{0}$ is set as the threshold for the neuron. . . . . . . . . . . . . . . . . . . 43

3.6 An illustrative example of the 'neuron addition to handle overlap' . . . . 45

3.7 An illustrative example of the 'parameter update strategy to handle novelty' 47

3.8 Illustrative examples of the 'parameter update strategy to handle overlap' 48

3.9 A flow chart depicting a single epoch of the learning algorithm for the

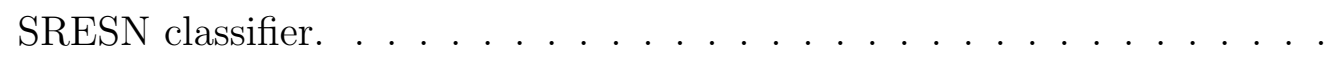

3.10 Effect of modulation factor $(\lambda)$ on the (a) Overall training and testing accuracy, (b) Number of neurons added by the SRESN classifier for the Ionosphere problem . . . . . . . . . . . . . . . .

3.11 Effect of neuron addition threshold in case of correct classification $\left(T_{C C}\right)$ on (a) Overall training and testing accuracy, (b) Number of neurons added by the SRESN classifier for the Ionosphere problem . . . . . . . . .

3.12 Effect of neuron addition threshold in case of misclassification on (a) Overall training and testing accuracy, (b) Number of neurons added by the SRESN classifier for the Ionosphere problem . . . . . . . . . . . .

3.13 Firing time of neurons for five random testing samples from Iris flower classification dataset . . . . . . . . . . . . . . . .

3.14 Two spike patterns with different spike times for which rank order learning would estimate equal weights . . . . . . . . . . . . .

4.1 Architecture of TMM-SNN . . . . . . . . . . . . . . .

4.2 The two stages of the learning algorithm for TMM-SNN. In the structure learning stage, the learning algorithm evolves the hidden layer neurons and updates the weights for synaptic connections between input and hidden layer neurons. In the output weights learning stage, the learning algorithm updates the weights for output layer neurons. . . . . . . . . . . 
4.3 An illustrative example of the activation based coding with three input

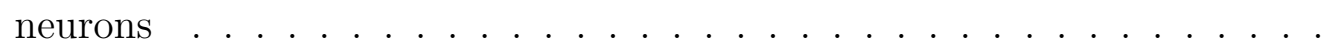

4.4 Effect of margin maximization strategy on the mean interclass margin for the response of (a) hidden layer neurons, (b) output layer neurons . . . . 77

4.5 Neuron growth history of TMM-SNN for the Ionosphere problem . . . . . 78

4.6 Effect of addition threshold on (a) training and testing accuracy of TMMSNN, (b) number of neurons added by TMM-SNN . . . . . . . . . . . 79

5.1 Architecture of a two layered spiking neural network with a meta-neuron. The input and output layer consists of $m$ and $n$ spiking neurons, respectively. The network is fully connected between the input and output layer. Further, the input layer neurons are also connected to the meta-neuron, which provides the meta-neuron access to the local information present in the input spike train on a particular synapse. The meta-neuron also has access to the global information (synaptic weights) stored in the network, represented by the oblique arrow from the meta-neuron across the network. 93

5.2 Neuron growth history of the learning algorithm for the Ionosphere problem 106

5.3 Effect of novlety threshold $\left(\alpha_{n}\right)$ on the (a) training and testing accuracy, (b) number of neurons required to approximate the decision function . . 107

5.4 Effect of learning rate $\left(\alpha_{s}\right)$ on the (a) training and testing accuracy, (b) number of neurons required to approximate the decision function . . . . . 108

6.1 Structure of the neuromorphic implementation of OMLA . . . . . . . 120

6.2 Structure of the simulator . . . . . . . . . . . . . . . . . . 122

6.3 Single iteration of CORDIC computations . . . . . . . . . . . . 124

6.4 Structure of the strategy selector; this component selects the appropriate learning strategy for a given input spike pattern . . . . . . . . . . . 125

6.5 Structure of meta-neuron simulator module . . . . . . . . . . . . . 127

6.6 Effect of precision on the performance of OMLA-RBP . . . . . . . . . 131

6.7 OMLA performance (\%) for different noise levels (SNR) for the Ionosphere

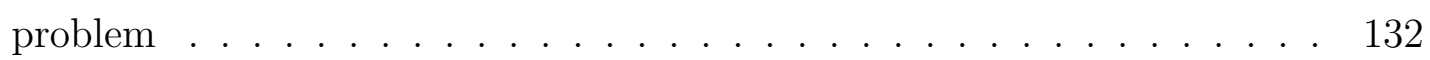




\section{List of Tables}

2.1 Summary of major algorithms discussed in the literature review . . . . . 32

3.1 Performance comparison of SRESN with existing spiking classifiers - Fisher Iris problem . . . . . . . . . . . . . . . . . . . . . 55

3.2 Performance comparison of the SRESN classifier with existing spiking classifiers - Wisconsin Breast Cancer problem. . . . . . . . . . . . . . . 57

4.1 Description of the data sets used for evaluation . . . . . . . . . . 80

4.2 The value for the parameter addition threshold $\left(\alpha_{a}\right)$ used for evaluating TMM-SNN on the benchmark data sets . . . . . . . . . . . . . . . . . . 81

4.3 Average execution per epoch of the SRESN classifier . . . . . . . . . . 82

4.4 Performance comparison of TMM-SNN with SpikeProp, SWAT and SRESN for benchmark problems . . . . . . . . . . . . . . . 83

4.5 Description of the benchmark data set used for the comparison between eSNN and TMM-SNN . . . . . . . . . . . . . . . . . 86

4.6 Performance comparison of TMM-SNN and eSNN . . . . . . . . . . . . 87

4.7 Description of the benchmark data set used for the comparison between ASA and TMM-SNN . . . . . . . . . . . . . . . . . 87

4.8 Performance comparison of TMM-SN and ASA $\ldots \ldots \ldots$

5.1 Description of the data sets used for evaluation . . . . . . . . . . 110

5.2 Parameter values for novelty threshold $\left(\alpha_{n}\right)$ and learning rate $\left(\alpha_{s}\right)$ for benchmark data sets used for comparison . . . . . . . . . . . . . . 111

5.3 Performance comparison of OMLA with OSNN and the Online SRESN . 112

5.4 Performance comparison of OMLA with SpikeProp, SWAT and TMM-SNN 114 
6.1 Truth table for strategy selection in strategy selector . . . . . . . . 126

6.2 Performance comparison of OMLA-RBP with OMLA-DP . . . . . . . . 133 


\section{Chapter 1}

\section{Introduction}

\subsection{Motivation and Challenges}

Many important concepts in the area of artificial neural networks have been inspired by new findings in the area of neuroscience. This has helped in the development of more biologically plausible architectures and learning mechanisms for artificial neural networks. In this regards, an important observation was made by E.D. Adrian that the biological neurons use the frequency of spikes to encode the intensity of the stimulus [1]. This form of neural coding is termed as rate coding and led to the development of artificial neurons with a continuous response. The continuous response of these neurons is analogous of the mean firing rate of a neuron in the brain.

Recent works in the area of neuroscience, have shown that the biological neurons utilize the precise time of spikes to transmit information in the brain [2, 3]. In [2], it has been highlighted that the information about a visual stimulus reaches the brain from the retina in less than $50 \mathrm{~ms}$, which limits the time available for processing and transmitting the information at the intermediate stages to lower than $10 \mathrm{~ms}$. This implies that the information is transmitted between stages using very few spikes which indicate that the information is represented in the brain using the times of spikes. Similarly, in [3] it has been shown that the relative timing of the first spikes generated by the individual afferents conveys information about the direction of force applied by the fingertip and the shape of the surface in contact with the fingertip. This has led to the development of an artificial neuron that uses discrete events (spikes) in time for transmitting information. 


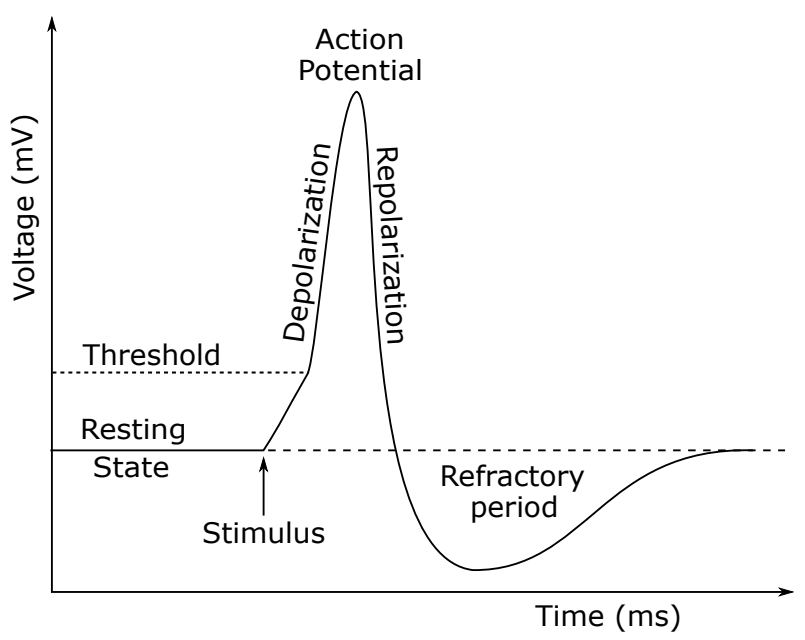

Figure 1.1: Different phases of an action potential ${ }^{1}$

A spike is a simplified representation of an action potential in a biological neuron. Figure 1.1 shows the different phases of an action potential in a neuron. In the absence of any stimulus, the neuron is in a resting state, and the membrane potential of the neuron in this state is termed as the resting potential. When an external stimulus is applied, the neuron starts depolarizing i.e. the membrane potential of the neuron starts growing. This phase is termed as the depolarization phase. When the membrane potential of the neuron reaches a threshold value, it rises sharply. An action potential is said to have occurred when the membrane potential reaches the peak value. After generating an action potential, the membrane potential of the neuron decays abruptly (repolarization phase) and undershoots the resting potential. This phase is termed as the refractory period. In this period, the neuron exhibits a subdued response to any external stimulus and its membrane potential slowly returns to the resting potential.

It has been shown in the literature that the amplitude of an action potential is not representative of the strength of the stimulus [4]. The strength of the stimulus only determines the occurrence or non-occurrence of an action potential. Similarly, a spike, which represents the occurrence or non-occurrence of an action potential, is a binary event in time. The neuron model used to simulate a spiking neuron determines how closely that neuron reproduces the characteristics of an action potential. The HodgkinHuxley neuron model [5] describes the different ionic mechanisms underlying an action potential, although it is computationally expensive. Several computationally tractable 
spiking neuron models have been proposed in literature like leaky integrate-and-fire model [6], Izhikevich neuron [7], etc. The output of all spiking neuron models is discontinuous in time, unlike neuron models for previous generations of neural networks that have a continuous output. Due to this discontinuity, it is hard to use existing learning techniques for spiking neurons. The similarities between spiking neurons and biological neurons have motivated researchers to study the plasticity mechanisms observed in the brain to develop efficient learning techniques for spiking neurons.

Spike Timing Dependent Plasticity (STDP) [8] is one of the widely studied plasticity mechanisms observed in the brain. It uses the difference between the times of pre- and postsynaptic spikes to update the synaptic weight of a given neuron. Thus, STDP is a local learning mechanism, as it does not take into account the information on other synapses (global information) while updating the synaptic weights of a given synapse. The local learning mechanism of STDP acts as a feedback loop for potentiated synapses, thereby leading to their further potentiation [9]. This can result in the creation of functionally unbalanced dendritic compartments in the brain, in the absence of any counterbalancing mechanism. Also, the local nature of STDP may lead to convergence issues while estimating the synaptic weights of a spiking neuron [10]. Thus, there is a need to develop heterosynaptic learning techniques, which can utilize the local and global information, for updating the weights of spiking neurons.

Next, we will discuss the issues in the development of learning algorithms for a network of spiking neurons, called as Spiking Neural Network (SNN). An important issue in the development of learning algorithms for SNNs is choosing the appropriate network architecture for a given problem. In this thesis, we will focus specifically on pattern classification problems in a supervised learning framework. For this purpose, a rank order learning based evolving Spiking Neural Network (eSNN) has been developed for pattern classification problems in [11].

An eSNN starts with zero neurons and evolves the network architecture automatically. It uses rank order learning to initialize the parameters (weights and threshold) of a newly added neuron and to update the parameters of existing neurons. Rank order learning is based on the assumption that the most important information about an input spike pattern is present in the first spikes generated by the input neurons [12]. It utilizes the 
order in which first spikes are generated by the input neurons and ignores the precise times of the spikes. To update the weights of existing neurons, rank order learning averages the rank order of the current inpit spike pattern and the weights of existing neurons in the network. This may result in loss of knowledge acquired by the network from the past spike patterns when two spike patterns with similar order of spikes but different times of spikes are presented to the network. Also, the loss of knowledge may lead to addition of more neurons to the network. In [12], a dynamic evolving Spiking Neural Network (deSNN) has been developed that extends rank order learning to use information present in multiple spikes. It uses the order of first spikes generated by the input neurons to initialize the weights of a newly added neuron, and subsequent spikes are used to fine-tune the weights. Hence, rank order learning uses the global information in the network, but it only considers the order in which presynaptic neurons generate first spikes to initialize the weights of a neuron. It ignores the information present in the precise times of the first spikes. As a result, it is difficult to use rank order learning for training a neuron to generate precisely timed spikes. Further, both eSNN and deSNN use a two layered SNN to approximate the functional relationship between input spike patterns and the corresponding class labels.

For online learning, it is important to develop learning algorithms in which the neuron parameters are initialized such that the neuron generates precisely timed spikes. Hence, there is a need to develop a learning algorithm which can evolve the network architecture and update the synaptic weights on-the-go.

The major challenges in the area of spiking neural networks for pattern classification problems, that are addressed in this thesis are:

- Development of a learning algorithm that takes into account the local and global information to closely represent the relationship between the input and output spike patterns.

- Evolve the network architecture automatically to determine the appropriate network structure for a given problem.

- Development of learning algorithms that can learn from input spike patterns in an online framework. 


\subsection{Objectives of this Study}

The primary motivation is to develop efficient learning algorithms for SNNs that can be used for pattern classification problems. Based on this, the three main objectives of this thesis are:

- Heterosynaptic plasticity: For a given input and output spike pattern, each input neuron contributes a different amount of postsynaptic potential towards the spikes generated by the output neuron. Further, when the desired output consists of multiple spikes, any weight update affects the times of all the spikes generated by the output neuron. Therefore, it is essential to consider the local information on a synapse as well as the global information in the network to accurately capture the functional relationship between the input and output spike patterns. This thesis focuses on the development of such heterosynaptic learning techniques that can utilize the locally and globally available information to estimate network parameters such that the output neuron generates multiple spikes at precise times.

- Evolving network architecture: There is a need to develop evolving learning algorithms for projecting the input spike patterns to a suitable higher dimensional space which may provide better separability between different classes. This requires formulation of both effective rules for neuron addition and accurate techniques for initializing neuron parameters. For a pattern classification problem, it is also important to project the higher dimensional representation of the input spike patterns back to a lower dimensional output space with as many neurons as the number of classes. This helps in achieving improved generalization performance and learning using a compact network structure.

Thus, the three important issues in development of evolving learning algorithms for SNNs to handle pattern classification problems are as follows:

- When to add a neuron: A learning algorithm should be able to determine if the knowledge stored in the network is sufficient to approximate the current input spike pattern. This helps the learning algorithm to decide when a neuron should be added to the network. 
- How to add a neuron: The initialization mechanism for the parameters of a newly added neuron determines how accurately the network learns a given spike pattern. The existing techniques for initializing the weights of a newly added neuron may not be suitable for training a neuron to generate precisely timed spikes. This may result in the addition of a higher number of neurons to the network. Thus, it is essential to develop techniques for initializing neuron parameters that ensure generation of precisely timed spikes.

- Projection from a higher dimension to lower dimension: Existing evolving learning algorithms for pattern classification problems employ two layered SNNs and store the class association of the neurons in the output layer to determine the predicted class label for a given input spike pattern. By projecting the response of hidden layer neurons to a lower dimensional output space, the predicted class label can be easily determined. Further, this may also improve the generalization performance of the network.

- Online Learning: For many real world problems, data is generated continuously in real time and can not be stored for offline processing. Thus, there is a need to develop learning algorithms that can learn from the input spike patterns in an online manner. An online learning algorithm for pattern classification problem should be able to select autonomously its learning strategies for each input spike pattern such that over-training is avoided and higher generalization is achieved. Based on the information present in the input spike pattern with respect to the knowledge stored in the network, the learning algorithm should select one of the following strategies:

- Evolve the network structure to learn the new knowledge in the input spike patterns.

- Update the synaptic weights to fine-tune the stored knowledge.

- Discard the spike pattern from the learning process to avoid over-training.

\subsection{Major Contributions}

The major contributions of this thesis are: 
- Meta-neuron and its learning rule: This thesis describes the architecture of the two layered SNN with a meta-neuron that facilitates the use of both local and global information to update the weights of a neuron. The concept of meta-neuron is inspired by the heterosynaptic plasticity induced by astrocytes in the brain $[13$, 14, 16]. The astrocytes are biological cells that form an envelope around synapses in the brain. This allows astrocytes to intercept and modulate the information transmitted through a particular synapse. Further, astrocytes can envelope a large number of synapses simultaneously [15]. This allows astrocytes to access the local as well as global information in the network for modulating the plasticity of synapses in the brain.

In the SNN with a meta-neuron, the meta-neuron is connected to the synapses between input and output neurons. It can intercept the individual activities of the input neurons (local information) and has access to the weights of connected synapses (global information). The meta-neuron utilizes this information to compute the weight sensitivity modulation factor of a given synapse. The meta-neuron based learning rule employs the weight sensitivity modulation factor of a synapse and the postsynaptic potential of a neuron to update the synaptic weights. The weights of a postsynaptic neuron are updated using the meta-neuron based learning rule, such that its output spike is shifted precisely in the desired direction.

- Online meta-neuron based learning algorithm: Using the meta-neuron based learning rule, an Online Meta-neuron based Learning Algorithm (OMLA) is developed that evolves the network architecture and updates the synaptic weights for a pattern classification problem in a supervised learning framework. The learning algorithm uses different strategies to learn each input spike pattern in a single presentation. It can choose to employ either 'neuron addition strategy' or 'delete sample strategy' or 'parameter update strategy'. The 'neuron addition strategy' is selected when the information in the current input spike pattern can not be approximated by the knowledge stored in the network. The OMLA employs a meta-neuron with memory to store those spike patterns that were previously used by the OMLA to add a new neuron to the network. The spike patterns in meta-neuron memory are used to update the weights of the newly added neuron such that it closely ap- 
proximates the past knowledge stored in the network. The learning algorithm uses the 'delete sample strategy' to delete those spike patterns from the learning process that are similar to the already learned spike patterns. The "parameter update strategy' is chosen when the information present in the current input spike pattern is not enough to add a neuron but the spike pattern contains some information different from the knowledge stored in the network. The weights of a neuron are updated using the meta-neuron based learning rule to produce precise shifts in the spike times of a postsynaptic neuron.

- Two stage margin maximization spiking neural network: The Two Stage Margin maximization Spiking Neural Network (TMM-SNN) has been developed keeping in mind that an important property of biologically observed learning mechanisms is that they rely only on the locally available information to update the weights of a given synapse. TMM-SNN employs a three layered SNN with an evolving hidden layer. The learning algorithm for TMM-SNN employs a two stage learning mechanism. In the first stage (structure learning stage), the learning algorithm determines the appropriate number of hidden neurons for a given problem and also estimates the parameters (weights and thresholds) for the hidden neurons. The learning algorithm employs two different learning strategies in this stage, namely 'neuron addition strategy' and 'margin maximization strategy for structure learning'. The 'neuron addition strategy' is used to evolve the hidden layer completely in the first epoch. The weights of the newly added neuron are initialized using a new Activation Based Coding ( $\mathrm{ABC}$ ) technique, developed in this thesis. The ABC technique uses the locally available information present in multiple spikes generated by the presynaptic neurons to initialize the synaptic weights of a newly added neuron. The weights and thresholds of the newly added neuron are initialized such that it fires at a precise time for the current input spike pattern. The weights of the hidden neurons are updated using the 'margin maximization strategy for structure learning'. This strategy uses only the locally available information to update the synaptic weights such that the separation between the spike times of the interclass and intraclass hidden neurons is maximized. The weights of the hidden neurons are updated for multiple epochs, until the average separation for all the training spike 
patterns converges to a maximum value.

The second stage of the learning algorithm is termed as the output weights learning stage. In this stage, the learning algorithm updates the weights of output layer neurons without modifying the parameters estimated in the first stage. The weights of the output layer neurons are updated using a "margin maximization strategy for output weights learning' similar to the update strategy used in the first stage. This strategy also relies only on the locally available information to update the weights of output layer neurons such that the separation between the spike times of the interclass and intraclass output neurons is maximized. The update mechanism in this stage is also used for multiple epochs until the average separation for all the training spike patterns converges to a maximum value.

- Self-regulating evolving spiking neural classifier: The Self-Regulating Evolving Spiking Neural (SRESN) classifier employs a modified form of rank order learning to update the synaptic weights in the network. Rank order learning has been developed based on the observations pertaining to the visual processing pathway. It has been shown in [2] that the intermediate stages in the visual processing pathway have less than $10 \mathrm{~ms}$ to process and transmit information to the subsequent stages. This implies that the information is transmitted between stages using few spikes which indicated the existence of an efficient encoding mechanism in the brain. Based on these observations, rank order learning has been developed as a learning mechanism that relies on the order of presynaptic spikes.

The SRESN classifier employs rank order learning to evolve the network architecture and simultaneously update the synaptic weights. It uses a different learning strategy for each input spike pattern. The appropriate learning strategy for a given spike pattern is selected according to the times of spikes generated by the output layer neurons. This allows the learning algorithm to accurately estimate the information present in the current input spike pattern with respect to the knowledge stored in the network. The learning algorithm can choose either 'skip sample strategy' or 'neuron addition strategy' or 'parameter update strategy' for a given input spike pattern. It chooses the 'skip sample strategy' when the information present in 
the current input spike pattern is similar to the information already stored in the network. The 'neuron addition strategy' is chosen when the current input spike pattern contains a significant amount of new information. The weights of a newly added neuron are initialized using rank order learning in a feature-wise manner. This allows the SRESN classifier to avoid non-contributing weights and achieve better discriminability between the input spike patterns. The threshold of the newly added neuron is initialized such that it fires at a precise time for the current input spike pattern. The learning algorithm chooses the 'parameter update strategy' when the information present in the current input spike pattern is not enough to add a neuron, but it contains some information which has not been learned by the network from previous spike patterns. In this strategy, the learning algorithm selectively updates the synaptic weights in the network using rank order learning in a feature-wise manner. The selective update mechanism helps in avoiding overfitting and preventing the loss of knowledge acquired from the past spike patterns learned by the network.

\section{- Hardware implementation of the online meta-neuron based learning algo-}

rithm: A neuromorphic implementation of the OMLA using a Field-Programmable Gate Array (FPGA) is described. The spiking neurons in the SNN are modeled using the spike response function [17] that is simulated in hardware using COordinate Rotation DIgital Computer (CORDIC) circuit [18]. The design uses a pipelined implementation of the CORDIC circuit which allows calculation of the postsynaptic potential of the neuron at a particular time instant in a single clock cycle.

\subsection{Organization of the Thesis}

This thesis is organized as follows:

- In chapter 2, a review of the existing spiking neuron models and the different learning algorithms for SNNs are presented. The existing learning algorithms for SNNs have been classified according to their underlying inspiration.

- Chapter 3 describes the architecture and learning algorithm of the Self-Regulating 
Evolving Spiking Neural (SRESN) classifier. The SRESN classifier employs different heuristic learning strategies for effectively approximating the information in the input spike patterns. It can choose to add a neuron or update the network parameters or skip learning a sample. It uses rank order learning in a feature-wise manner to initialize the parameters of a newly added neuron and update the synaptic weights of existing neurons. This helps the SRESN classifier in avoiding non-contributing synaptic weights for problems with a large number of features. Further, the parameter initialization mechanism in the SRESN classifier ensures that the newly added neuron fires at a precise time for the current input spike pattern. The working of the SRESN classifier is described using the Ionosphere problem from the UCI machine learning repository. This study is used to demonstrate the effects of different algorithm parameters on the performance of the SRESN classifier. Based on this study, certain guidelines are provided for setting the various parameters. This chapter also discusses performance evaluation results of the SRESN classifier for the problems of Iris flower classification and Wisconsin breast cancer problem. The results of performance evaluation are compared with the performance of other existing learning algorithms for SNNs, namely, SpikeProp [17], multi-spiking neural network [19], multi-spike learning [20], synaptic weight association training [10] and evolving spiking neural network [11].

- Chapter 4 describes the architecture and learning algorithm of the Two stage Margin Maximization Spiking Neural Network (TMM-SNN) for a three layered SNN. The learning algorithm for TMM-SNN has two different stages namely, structure learning stage and the output weights learning stage. In the structure learning stage, the learning algorithm evolves the hidden layer neurons and updates the synaptic weights of the hidden neurons. A new activation based coding scheme is developed in this thesis to initialize the weights of newly added hidden neurons. The activation based coding scheme relies only on the locally available information for initializing the weights of a neuron. The learning algorithm uses a new margin maximization learning rule that updates the synaptic weights such that the separation between the spike times of interclass neurons for all the training spike patterns is maximized. In the second stage, the learning algorithm uses the margin 
maximization learning rule to update the weights of output layer neurons.

The working of the TMM-SNN is described using the Ionosphere problem. Based on this study, guidelines for setting the algorithm parameter for TMM-SNN are also provided. The performance of TMM-SNN is evaluated on ten benchmark problems from the UCI machine learning repository [21], and the results of performance evaluation are compared with that of SpikeProp [17], synaptic weight association training [10] and the SRESN classifier. Performances of the different algorithms are compared using the one-way ANOVA test [22] followed by a pairwise comparison using the Fisher's least significant difference method [23]. The results of performance comparison clearly indicate that TMM-SNN performs better than the other algorithms within a 95\% confidence interval. Further, TMM-SNN employs a compact network architecture, and requires fewer epochs for convergence. The performance of TMM-SNN is also compared with recently proposed learning algorithms for SNNs, namely, evolving Spiking Neural Network (eSNN) [11] Accurate Synaptic-efficiency Adjustment (ASA) [24] using five benchmark problems from the UCI machine learning repository. The results of performance comparison clearly show that TMM-SNN performs significantly better than both these algorithms.

- Chapter 5 describes the concept of meta-neuron in a two layered spiking neural network. The concept of meta-neuron is inspired by the heterosynaptic plasticity induced by astrocytes in the brain. A meta-neuron based learning rule is developed that utilizes the local and global information in the network to update the synaptic weights of an output neuron such that its spike time is precisely shifted in the desired direction.

This chapter also describes the Online Meta-neuron based Learning Algorithm (OMLA) for SNNs that can be used to handle pattern classification problems. OMLA has been developed to learn from the input spike patterns in a single presentation. The learning algorithm uses different learning strategies to evolve the network architecture and simultaneously update the synaptic weights in an online framework. It uses the meta-neuron based learning rule to update the synaptic weights. The OMLA employs a meta-neuron with memory that is used to ensure 
a better approximation of past knowledge when a new neuron is added to the network. The working of the OMLA is illustrated using the Ionosphere problem from the UCI machine learning repository. Based on this study, guidelines are presented for setting the algorithm parameters of OMLA. The performance of OMLA is statistically compared with other existing online learning algorithms for SNNs using the one-way ANOVA test [22], followed by a pair-wise comparison using the Bonferroni test $[25,26]$. The results of performance analysis clearly depict that the OMLA performs better than the other learning algorithms. The performance of OMLA is also compared with that of other existing batch learning algorithms for SNNs. The results of performance comparison show that OMLA performs better than other algorithms using a compact network architecture. Further, OMLA requires a single epoch for learning whereas the other algorithms require multiple epochs.

- Chapter 6 presents a neuromorphic implementation of the OMLA using a FieldProgrammable Gate Array (FPGA). The spiking neurons in hardware are modeled using the spike response function and the postsynaptic potential of a spiking neuron is computed using the CORDIC circuit. A study is conducted using the Ionosphere problem from the UCI machine learning repository to analyze the impact of reduced precision for synaptic weights and noise on the performance of the neuromorphic implementation of the OMLA. The proposed implementation is also evaluated on benchmark problems from the UCI machine learning repository using reduced precision for synaptic weights. The results of performance evaluation indicate that the neuromorphic implementation of OMLA is able to emulate its software implementation.

- Chapter 7 summarizes the conclusions from this thesis and proposes several directions for future works. 
Chapter 1. Introduction 


\section{Chapter 2}

\section{A Review of Literature on Spiking Neural Networks}

Spiking neural networks are termed as the third generation of artificial neural networks [27] as they are significantly different from the previous generations of artificial neural networks. Further, it has been shown that a network of spiking neurons can mimic any feedforward sigmoidal neural network [28] and possesses greater computational capacity in comparison to a network of sigmoidal neurons [29]. Spiking Neural Networks (SNNs) are inspired by the developments in the area of neuroscience, which have highlighted that biological neurons employ the precise time of spikes for information transmission in the brain $[2,3]$. This similarity between SNNs and biological neural networks has motivated researchers to study the behavior of biological neurons for developing architectures and learning mechanisms for SNNs.

In this chapter, a review of the existing concepts related to the architecture and learning algorithms for spiking neural networks is provided. The spiking neuron models used in SNNs have been developed based on the physiological behavior of biological neurons. The commonly employed spiking neuron models in SNNs are integrate-andfire neuron [6], leaky integrate-and-fire neuron [6], Izhikevich neuron, etc. These neuron models employ a temporal signal with binary events to process and transmit information.

The structural and functional aspects of the brain have inspired the development of different SNN architectures like polychronous spiking neural networks [30] and NeuCube [31]. Polychronous SNNs employ synapses with varying delays to store time-locked spike 
patterns in the network. Several learning algorithms for polychronous SNNs have been developed that adapt the synaptic delays to generate learned spike patterns [32]. In NeuCube, a 3-dimensional spiking neural network with recurrent connections is used to learn spatio-temporal spike patterns. The neurons in the 3-dimensional SNN are topologically arranged to replicate the functional areas of the brain. A NeuCube can be trained using one of the existing learning mechanisms for adapting synaptic weights in SNNs. In this thesis, the focus is on the development of learning techniques for SNNs that update synaptic weights.

Based on the underlying inspiration, the learning algorithms for estimating the synaptic weights in spiking neural networks can be broadly classified into the following categories:

- Gradient based learning algorithms: The general idea in gradient based learning algorithms is to minimize an error function based on the desired and actual network output.

- Spike timing dependent plasticity based learning algorithms: Spike Timing Dependent Plasticity (STDP) [8,33] is considered to be the phenomenon used by biological neurons for the adaptation of synaptic weights. Different models of STDP have been used to develop learning mechanisms for SNNs.

- Rank order learning based learning algorithms: Rank order learning [12] assumes that the information is encoded in the order of first presynaptic spikes. The fast information transmission capabilities of rank order learning have inspired the development of many learning algorithms.

- Spike convolution based learning algorithms: To overcome the problems caused by the discontinuity in SNNs, several learning algorithms for SNNs convolve the original spike based signals with a continuous kernel function $[34,35]$. The generated continuous functions are used to develop a learning algorithm for SNNS. 


\subsection{Neuron Models}

The SNNs employ neuron models inspired by the physiological operation of a biological neuron. The physiology of an action potential is accurately described by the HodgkinHuxley model [5]. It reproduces all the stages of an action potential that were described in Section 1.1. But, the high computational requirements of the Hodgkin-Huxley model render it unsuitable for simulating SNNs on a large scale. To overcome these issues, several mathematically tractable spiking neuron models have been developed which reproduce the different aspects of the Hodgkin-Huxley model to varying degrees of accuracy. In this section, a description of the commonly employed neuron models is provided.

\subsubsection{Leaky Integrate-and-Fire Model}

The Leaky Integrate-and-Fire (LIF) [6] neuron model is a highly simplified version of the Hodgkin-Huxley model. It reproduces the depolarization phase of an action potential but, the repolarization phase and refractory period are not implicitly reproduced by the LIF neuron model. The membrane potential of the neuron is explicitly reset after generating a spike to model the repolarization phase. The LIF neuron model employs an absolute refractory period, during which the membrane potential of the neuron is fixed at the resting potential.

The LIF neuron model employs an electrical circuit with a resistance $(R)$ and a capacitor $(C)$ to emulate the membrane potential of a neuron. The electrical circuit is shown in Figure 2.1. The current $I(t)$ in the figure represents the presynaptic input current at

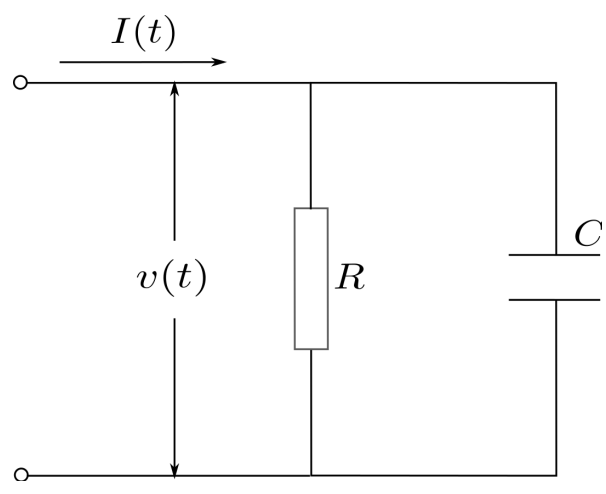

Figure 2.1: An RC circuit used to model a leaky integrate-and-fire neuron 
time $t$. The current causes voltage to build up across the capacitor, which represents the membrane potential $(v(t))$ of a neuron. Once the voltage across the capacitor crosses a certain threshold value, the neuron generates a spike and the potential of the neuron is reset to resting potential. This neuron model is termed as the leaky integrate-and-fire model because, in the absence of any presynaptic current, the voltage across the capacitor decays to zero due to the current induced by the capacitor in the $R C$-circuit.

The dynamics of the $R C$-circuit in Figure 2.1 are described as follows

$$
I(t)=\frac{v(t)}{R}+C \frac{d v}{d t}
$$

The input current $I(t)$ has two components viz. the current through the resistance $R$

and the current that charges the capacitor $C . \frac{v(t)}{R}$ is the component of current that goes through the resistance $R$ and $C \frac{d v}{d t}$ is the component of current that charges the capacitor. From Equation (2.1), the membrane potential of the neuron is given as

$$
\tau_{m} \frac{d v}{d t}=R I(t)-v(t)
$$

where $\tau_{m}$ is the time constant of the circuit. Lets assume that the simulation begins at $t=t_{0}$ and $v\left(t_{0}\right)=v_{r}$, where $v_{r}$ is the resting potential. Based on this initial condition the solution of the Equation (2.2) is given as

$$
v(t)=v_{r} \exp \left(-\frac{\left(t-t_{0}\right)}{\tau_{m}}\right)+\frac{1}{C} \int_{0}^{t-t_{0}} \exp \left(-\frac{s}{\tau_{m}}\right) I(t-s) d s
$$

A generalization of the leaky integrate-and-fire neuron that does not allow any leakage of current at the capacitor is termed as an integrate-and-fire neuron.

\subsubsection{Spike Response Model}

In comparison to the leaky integrate-and-fire neuron model, the Spike Response Model (SRM) [6] is capable of modeling an action potential more accurately as it implicitly models the refractory period. Assuming the last spike by the postsynaptic neuron was 


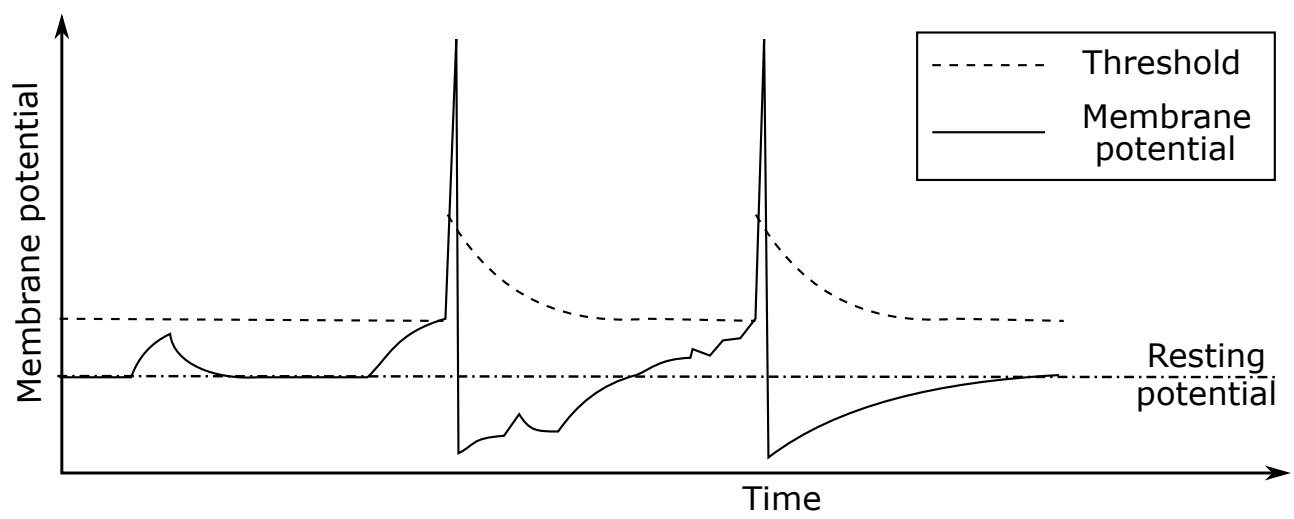

Figure 2.2: Membrane potential of a neuron modeled using the spike response model

generated at $\hat{t}$, the SRM describes the membrane potential $(v(t))$ at time $t$ as

$$
v(t)=\eta(t-\hat{t})+\int_{0}^{\infty} \kappa(t-\hat{t}, s) I(t-s) d s
$$

where the functions $\eta($.$) and \kappa($.$) are termed as the kernel functions. The function \eta($. determines the shape of an action potential. This function models the refractory period of the spiking neuron. The function $\kappa($.$) determines the response of the neuron to an$ input current $I(t)$. The function $\kappa($.$) is used to model the difference in the effects of$ input current on the membrane potential of a neuron during and after the refractory period. During the refractory period, a given input current will result in a smaller change in the membrane potential of the neuron. After the refractory period, the same input current will produce a normal change in the membrane potential of the neuron. Besides the $\eta($.$) and \kappa($.$) functions, the SRM employs a dynamic threshold function, represented$ by $\theta(t-\hat{t})$. The threshold of the neuron is maximum immediately after a spike and it slowly decays to the normal value.

Figure 2.2 shows the response of a spiking neuron modeled using SRM for a time varying input current. It can be observed from the figure, that the threshold of the neuron is not constant. It depends on the time of the last spike generated by the neuron.

The model of SRM defined in Equation 2.4 describes the membrane potential of a neuron for a time-varying input current. It can also be used to describe the membrane potential of a neuron for input current induced by presynaptic spikes. In this case, the time course of the current induced by the presynaptic spike is represented by an $\alpha($. 
function. Different functions have been used in literature to model current induced by a presynaptic spike [6]. In this thesis, the current at time $t$ induced by a presynaptic spike at $t_{\text {pre }}$ is modeled as

$$
\alpha\left(t-t_{\text {pre }}\right)=\frac{t-t_{\text {pre }}}{\tau} \exp \left(1-\frac{t-t_{\text {pre }}}{\tau}\right)
$$

Further, the neuron models used in this thesis do not have a refractory period. Therefore, the $\alpha($.) is used directly to represent the membrane potential of the neuron. This approach has been used in [17]. The function $\alpha($.$) defined in Equation 2.5$ is also referred to as spike response function in literature [17].

\subsubsection{Izhikevich Neuron Model}

Izhikevich neuron model [7] has been developed with the goal of replicating different spiking behaviors observed in the cortical neurons. It simulates a spiking neuron by modeling the movements of ions across the cell membrane. The following equations describe the dynamics of the Izhikevich neuron model:

$$
\begin{aligned}
& d v / d t=0.04 v^{2}+5 v+140-u+I \\
& d u / d t=a(b v-u)
\end{aligned}
$$

where $v$ represents the Postsynaptic Potential (PSP) of the neuron, $u$ represents the membrane recovery variable and $I$ is the current induced by a presynaptic spike. The membrane recovery variable models the movement of $\mathrm{Na}^{+}$and $\mathrm{K}^{+}$ions across the cell membrane. a determines the length of refractory period by controlling the recovery of $u$, and $b$ modulates the sensitivity of $u$ to the sub-threshold changes in $v$.

After generating a spike, the postsynaptic potential of the neuron $v$ and the recovery variable $(u)$ are reset as follows

$$
\begin{aligned}
& v=c \\
& u=u+d
\end{aligned}
$$


where $c$ and $d$ determine the values of $u$ and $v$ after reset. The Izhikevich neuron model is suitable for large scale simulation of cortical neurons when there is a need for replicating the intrinsic properties of biological cells.

Next, a description of different learning algorithms for SNNs will be provided.

\subsection{Learning Algorithms for Spiking Neural Networks}

In the earlier sections, different spiking neuron models for simulating SNNs are discussed. In this section, a review of the existing learning algorithms for SNNs is provided. The review is organized based on the general inspiration for different learning techniques.

In literature, SNNs have been used for addressing different problems like unsupervised learning, reinforcement learning, etc. In this section, we focus our discussion on the techniques that are developed for employing SNNs in a supervised learning framework.

\subsubsection{Gradient based Learning Algorithms}

The gradient based learning algorithms update the synaptic weights in the network so as to minimize a given error function. The concept of error minimization using gradient has been extensively used for updating synaptic weights in the previous generation of artificial neural networks. But, the calculation of gradient is not defined for SNNs at the time of spike; as a result, other error functions have been used for adapting synaptic weights in SNNs. The major gradient based learning algorithms for SNNs are:

- SpikeProp [17] extended the traditional error-backpropagation learning algorithms for previous generations of artificial neural networks to SNNs. It employs a three layered network with spiking neurons that are modeled using the spike response function. The desired output of the network is encoded using spikes at precise time instants. Based on this, SpikeProp uses an error function that computes the difference between the times of the desired and actual spikes generated by the output neurons. But, the discontinous nature of spiking neurons makes it difficult to compute the gradient of the error function. For this purpose, SpikeProp assumes that the postsynaptic potential changes linearly with time around the time of spike, thereby allowing calculation of gradient at the time of spike. 
Several extensions to SpikeProp have been developed that improve its convergence mechanism [36, 37, 38] and allow error-backpropagation using multiple spikes [20, 19]. SpikeProp and all its extensions are susceptible to a proper initialization of weights [39] and the 'silent neuron' problem [40]. In [39], it has been highlighted that using inhibitory weights to initialize the synaptic weights for SpikeProp causes surges in the learning process which may affect the convergence of the learning algorithm. The 'silent neuron' refers to the difficulty in calculating error gradient of a neuron that failed to spike during the learning process.

- Chronotron [41] is a gradient based learning algorithm for updating synaptic weights in a single layer of spiking neurons. It employs the Victor-Purpura distance metric [42] to estimate the distance between the actual and desired spike patterns. The distance between the actual and desired spike patterns is used as an error function to derive a gradient based learning rule for updating the synaptic weights.

- Tempotron [43] develops an iterative (batch) learning mechanism to update synaptic weights in a single layer SNN. The desired response of the output neuron is encoded according to the presence or absence of a spike; as a result, the learning algorithm is used for binary pattern classification problems. Suppose the two classes are represented as $\oplus$ and $\ominus$ and the desired response of the output neuron for a spike pattern from class $\oplus$ is to generate a spike. Conversely, the output neuron should not generate a spike when the input spike pattern is from class $\ominus$. Let, the time instant when the PSP reaches the maximum value be denoted by $t_{\text {max }}$, then the synaptic weight for a given synapse is updated in proportion to the PSP induced by the corresponding presynaptic neuron at $t_{\max }$. Thus, the synaptic weights for the presynaptic neurons undergo long term potentiation when the postsynaptic neuron does not generate a spike for an input spike pattern from class $\oplus$. Similarly, the synaptic weights are subjected to long term depression when the postsynaptic neuron generates a spike for an input spike pattern from class $\ominus$. Recently, the Tempotron learning rule has been extended to multi-class problems in $[44,45,46]$. 


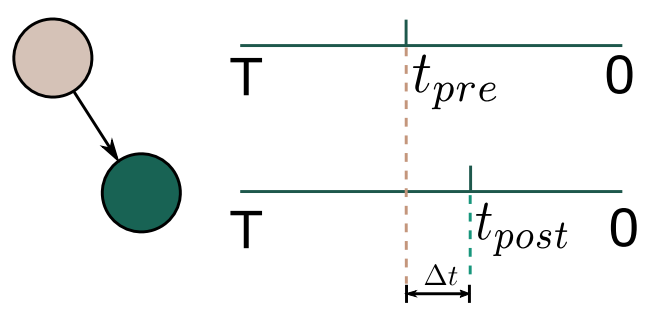

(a)

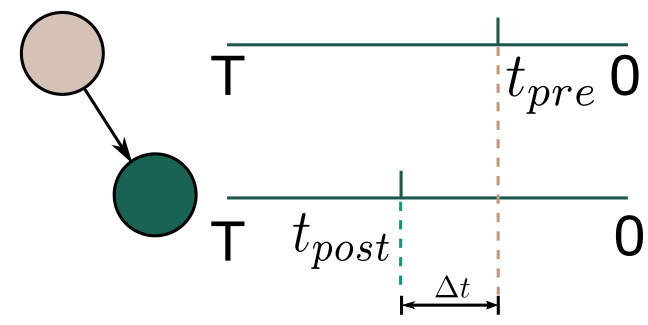

(b)

Figure 2.3: Examples of STDP causing (a) long term potentiation and (b) long term depression

\subsubsection{Spike Timing Dependent Plasticity Based Learning Algo- rithms}

Spike Timing Dependent Plasticity (STDP) $[8,33]$ is one of the most studied biologically plausible learning mechanism. It is a local learning rule that relies on the time of pre- and postsynaptic spike times to modulate the plasticity of a given synapse. Figure 2.3 shows two examples where STDP would result in long term potentiation and long term depression. In the figure, $t_{\text {pre }}$ and $t_{\text {post }}$ represent the spike times of the pre- and postsynaptic neuron. Figure 2.3a shows that, when a presynaptic spike occurs before the postsynaptic spike, STDP causes long term potentiation. Similarly, Figure 2.3b shows that, when a presynaptic spike occurs after the postsynaptic spike, the weight of the synapse between the pre- and postsynaptic neuron undergoes long term depression according to STDP.

The change in the synaptic weight $(\Delta w)$ of the connection between the pre- and postsynaptic neurons depends on the temporal difference $(\Delta t)$ between the time of postsynaptic and presynaptic spike. It is expressed using an STDP learning window shown in Figure 2.4. The change in weight is higher when the difference between the time of spike for the pre- and postsynaptic neurons is smaller. As the value of $\Delta t$ increases, $\Delta w$ becomes smaller.

STDP has been the motivation for the development of many learning algorithms in the literature. The important STDP based learning algorithms for SNNs are described below:

- Remote Supervised Method (ReSuMe) $[47,48,49]$ is one of the first learning algorithms for a SNN, that is based on STDP. ReSuMe uses the Widrow-Hoff learning 


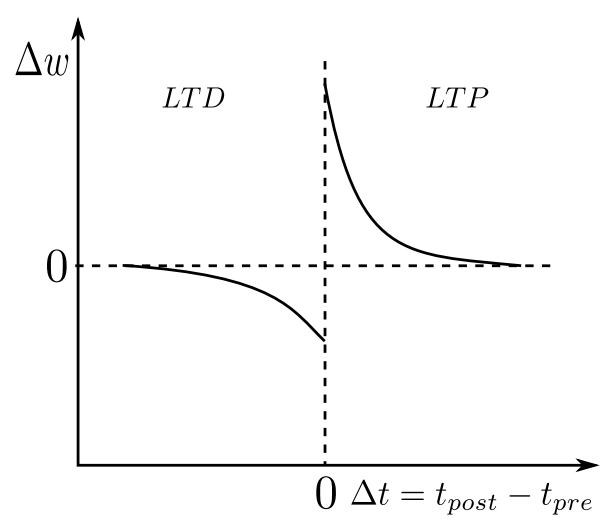

Figure 2.4: Learning window in STDP

rule to derive an update rule for a single layer of spiking neurons. It describes the Widrow-Hoff learning rule as a combination of a Hebbian and an anti-Hebbian learning mechanism. The Hebbian term of the learning rule models the correlation between the desired output signal and the input signal. The anti-Hebbian term models the correlation between the actual output and the input signal. The two learning mechanisms are expressed using the model of STDP described in [50] to derive a learning rule used for updating the synaptic weights in the network. Recently, ReSuMe has also been combined with error-backpropagation to train a multilayer SNN [51]. In $[52,53,54]$, the authors have extended the ReSuMe learning algorithm to adapt both weights and delays in a layer of spiking neurons.

- Synaptic Weight Association Training (SWAT) [10] uses a combination of STDP and the Bienenstock Cooper Munro (BCM) [55] learning rule to update the synaptic weights in the network. SWAT employs a three layered network but only the weights for output layer neurons are updated. The input layer is used to present spike patterns to the network, and the hidden neurons act as a frequency filter that detect different firing frequencies in the input data. The output layer consists of as many neurons as the number of classes. During training, SWAT uses a single training neuron connected to all the hidden neurons whose weights are adapted using STDP. The learning algorithm tracks the total class-wise changes in weights of the teacher neuron in each epoch. At the end of each epoch, the learning algorithm maps these weights to the output layer neurons. This process is repeated until the output 
frequency of the teacher neuron stabilizes. It has been highlighted in literature that the local nature of STDP can result in the creation of overactive/inactive dendritic regions in the brain in the absence of any counterbalancing mechanism [9]. In this regards, the BCM learning rule is used to modulate the height of the plasticity window to ensure that the response of the training neuron converges.

- STDP has also been used to develop an online learning algorithm for a three layered SNN in [56]. The learning algorithm uses a combination of STDP and anti-STDP to update the weights of the output neurons. The weights of the hidden neurons are updated using a Hebbian-based unsupervised winner-take-all learning rule. The number of neurons in the hidden layer of the SNN is not fixed and is determined by the learning algorithm during the training process. Each hidden neuron is treated as a radial basis function neuron. When the learning algorithm adds a neuron, the center of the radial basis function neurons is set as the time of the first spike generated by that neuron for the current training spike pattern. The learning algorithm also prunes hidden neurons based on a pairwise temporal distance.

- Recently, an Accurate Synaptic-efficiency Adjustment (ASA) method has been proposed in [24] inspired by the fast information transmission mechanism observed during visual information processing [2]. The weight update in ASA is computed according to the normalized weight update due to STDP and the total error in the PSP of the postsynaptic neuron. This approach allows ASA to achieve faster convergence in comparison to other existing learning algorithms for SNNs.

\subsubsection{Rank Order Based Learning Algorithms}

Rank order learning is inspired by the faster processing times exhibited by the visual system $[2,57]$. The faster processing times imply that the information is communicated by the retina to the brain using very few spikes. Based on this observation, rank order learning has been developed which employs the order of first spikes generated by a group of presynaptic neurons. Figure 2.5 illustrates the application of rank order learning using a network with five presynaptic neurons and a single postsynaptic neuron. In the figure, $t_{1}, t_{2}, t_{3}, t_{4}$ and $t_{5}$ represent the time of first spikes generated by the presynaptic neurons. 
Using the time of spike, the ranks of each presynaptic neuron are equal to $3,1,2,4$ and 0 . Based on the determined ranks, the weights for the synaptic connections between the presynaptic and postsynaptic neurons are initialized as $\lambda^{3}, \lambda^{3}, \lambda^{2}, \lambda^{4}$ and $\lambda^{0}$ where $\lambda$ is termed as the modulation factor and is set in the interval $[0,1]$.

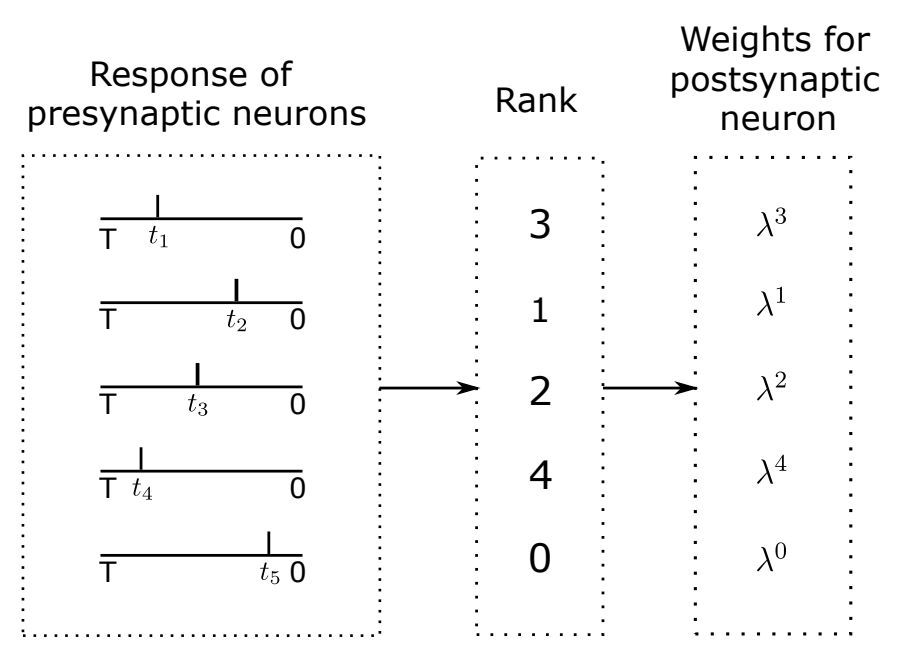

Figure 2.5: An illustrative example of the rank order learning using five presynaptic neurons and a single postsynaptic neuron

In $[58,59]$, rank order learning has been used for processing visual data like images, videos, etc. in an online framework. The architecture of SNN employed in [58] consists of four layers of spiking neurons with contrast sensitive neuronal maps in the second layer and orientation selective neuronal maps in the third layer. The first layer is used to present images to the network, and the fourth layer is used to determine the predicted class label. The number of neuronal maps in the fourth layer of the network is not fixed and is determined by the learning algorithm during training. The synaptic weights for the neurons in the fourth layer are updated in a supervised manner to learn the functional relationship between the input spike patterns and their class labels. In [59], a similar architecture is used but the output neuronal maps are connected to a single neuron which represents the predicted class. In [60], rank order learning is used for processing audio and visual information simultaneously in an online manner.

The online learning algorithms mentioned above utilize rank order learning to select the weights for newly added neuronal maps according to the time of first spikes generated by the presynaptic neurons. The information present in subsequent spikes is not taken 
into account while selecting the network parameters. Dynamic evolving Spiking Neural Network [12] have been developed to effectively exploit the information present in multispike signals. It uses rank order learning to select the synaptic weights appropriately based on the time of the first spike and subsequent spikes are employed to fine-tune the selected weights. Recently, rank order learning has been extended to take into account the precise time of spikes during learning in [61].

\subsubsection{Spike Convolution Based Learning Algorithms}

As described earlier, the discontinuous nature of spikes is problematic in using the existing techniques for earlier generations of artificial neural networks to train a network of spiking neurons. Several algorithms have been proposed in the literature that convolve the original spike pattern with a kernel function to convert the discontinuous spike signal into a continuous analog signal.

A Spike Pattern Association Neuron (SPAN) has been developed in [62], that convolve the input spike patterns, actual output spike patterns and the desired output spike patterns with a kernel function. The convolved signals are substituted in the WidrowHoff learning rule to derive a learning rule for a single leaky integrate-and-fire spiking neuron. In $[63,64,34,65]$, the SPAN algorithm has been extended to a network of spiking neurons. A similar convolution technique has also been used to optimize the parameters of an evolving SNN using particle swarm optimization in [66]. Different from SPAN, the Precise-Spike-Driven (PSD) [35, 67, 68] learning rule has been developed by convolving only the input spike pattern with a kernel function.

In literature, evolutionary techniques like particle swarm optimization [69] and quantum inspired evolutionary computing [70] have also been used for parameter estimation in SNNs.

\subsection{Applications}

Spiking Neural Networks (SNNs) can be used for all those applications where modern neural architectures are employed. This can be achieved by using techniques like population coding for converting the continuous data obtained in most real world application 
into spike trains.

An important advantage of SNNs with respect to modern neural architectures is that SNNs can be realized in hardware with smaller power requirements. The modern neural architecture like convolutional neural networks, autoencoders, etc. employ deep and complex network architecture to achieve great performance for real world applications. These neural network architectures impose high power requirements for practical applications. With the advent of low power neuromorphic chips like Neurogrid [97], TrueNorth [98], it is possible to realize large scale SNNs economically for practical applications.

Below, we discuss some of the examples where SNNs have been used for handling real world problems.

\subsubsection{Face detection and identification}

Spiking neural networks have been used in a supervised learning framework for the problems of face identification [71]. In this work, a three layered SNN, inspired by the architecture of primate visual system, is employed for recognizing faces. The network can detect faces in unseen images and is also resistant to variations in the image contrast.

\subsubsection{NeuCube Applications}

NeuCube is commonly trained using the principles of evolving spiking neural networks. In [72], a detailed discussion on the applications of evolving SNNs for a broad range of applications like visual pattern recognition, auditory pattern processing, taste recognition, ecological modeling, sign language recognition, etc. has been presented. This section describes some of the recent applications of NeuCube.

- Medical Data Analysis: The NeuCube architecture has been used to address many problems in the area of medical analytics because of its ability to handle spatio-temporal data efficiently. It has been used for modeling of weather and stroke data to study the effect of weather on stroke patients in different age groups $[73,74]$.

The NeuCube has also been used for modeling EEG data to study the functional changes in the brain of an opiate addict and a normal individual [75]. The study 
on opiate patients is also used to analyze the changes in the brain when the opiate patient is administrated some treatment. The NeuCube architecture has also been used to study EEG data recorded from epileptic patients [76] and Alzheimer's patients [77].

- Cow Movement Detection: In [78], a 3-dimensional eSNN model is trained to detect different cow gestures. The model uses AdaBoost to detect cows in an image. The detected cow body regions in the images are used to train a NeuCube to classify the behavior of cows into five different classes based on the activity of the cows. The five different activities are walking, running, jumping, standing and laying. The trained NeuCube model can effectively distinguish the different gestures performed by the cows.

\subsubsection{Applications in Energy Sector}

In [79], variational mode decomposition is used with SNNs to forecast the carbon price. The input features for SNNs have been generated using the intrinsic mode function components obtained by variational mode decomposition of the original carbon price data. Each intrinsic mode function component has been used to generate a different forecasting model. The results from different models have been combined to detect the variation in carbon price trends. The results indicate that SNN based forecasting models can accurately determine the changes in the trends of carbon price.

In [80], SNNs have been used for short term load forecasting. A SNN has been used to model the effects of different factors like temperature, humidity, day of the week, etc. on the short term load. The results clearly show that SNNs can effectively handle the historical data.

\subsection{Summary}

This chapter reviewed the different neuron models for spiking neurons and the major learning algorithms for SNNs. The learning algorithms for SNNs were classified into four different categories according to the learning technique used. The initial algorithms 
for SNNs were gradient based techniques, inspired by the effectiveness of these techniques for previous generations of neural networks like SpikeProp [17], Tempotron [43] and Chronotron [41]. Many algorithms for SNNs have been developed using the different biological plasticity mechanisms because of the closeness between biological neurons and spiking neurons.

Table 2.1 provides a summary of the different learning algorithms reviewed in this chapter. SpikeProp [17], Chronotron [41] and Tempotron [43] are the major gradient based learning algorithms for SNNs. It can be observed from the table that SpikeProp can be used for training a multilayer network. Further, it utilizes the local as well as global information in the network to update the synaptic weights. But, SpikeProp is susceptible to a proper initialization of weights [39, 36] and 'silent neuron' problem [40]. Chronotron and Tempotron are other gradient based learning algorithms that have been developed for updating the synaptic weights in a two layered SNN. Both these algorithms utilize only the locally available information to update synaptic weights in the network. ReSuMe [47], SWAT [10], online SNN proposed in [81], SPAN [34] and ASA [24] are STDP based learning algorithms that also rely only on the locally available information to update synaptic weights. In [60], a rank order learning based learning algorithm has been developed for SNNs that uses the global information in the network. But, rank order learning is based on the order of spikes and ignores the precise time of spikes.

Based on learning algorithms discussed in this chapter, the important issues in the existing learning algorithms that are addressed in this thesis are:

- Effectively utilize the local and global information in the network to update the synaptic weights.

- Determine the appropriate network architecture automatically based on the spike patterns presented during training.

- Development of online learning algorithms that require a single presentation of the training spike patterns for learning.

In the next chapter, a Self-Regulating Evolving Spiking Neural (SRESN) classifier is presented that employs heuristic learning strategies to evolve the network architecture and update the synaptic weights. 


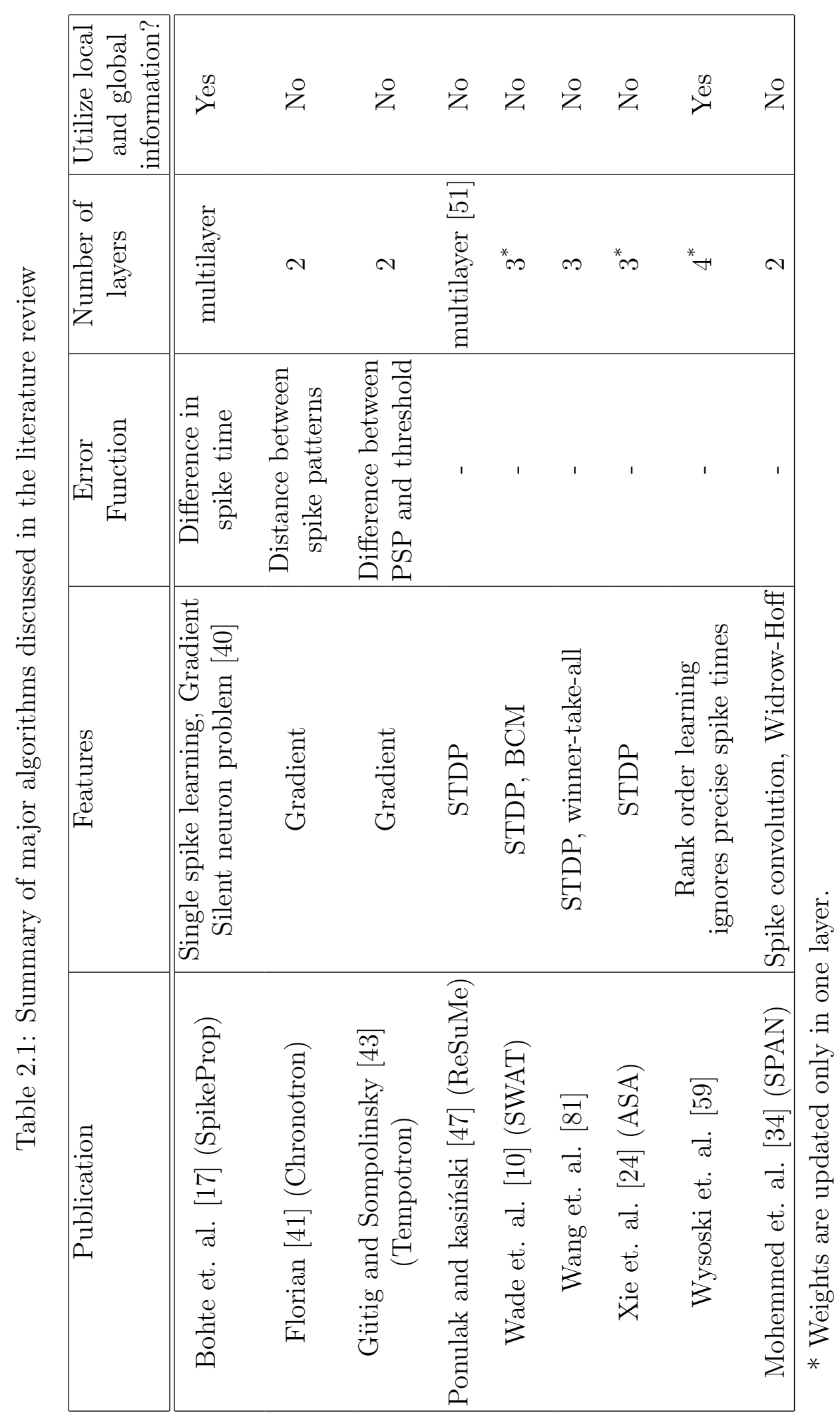


Chapter 2. Literature Review 


\section{Chapter 3}

\section{A Self-Regulating Evolving Spiking Neural Classifier}

In the previous chapter, a review of the existing learning algorithms for spiking neural networks was presented. This chapter describes a self-regulating evolving spiking neural classifier that evolves the network architecture and simultaneously updates the synaptic weights.

Recently, an evolving learning algorithm has been proposed for SNNs in [11]. It uses rank order learning to evolve the network architecture. The weights of existing neurons are updated using the average of the existing weights and the rank order for the current input spike pattern. This may result in an SNN with a higher number of neurons and loss of knowledge in the network when two spike patterns with similar order but considerable different spike times are presented to the network.

In this chapter, we develop a self-regulated learning algorithm for a two layered SNN, known as the Self-Regulating Evolving Spiking Neural (SRESN) classifier. The SRESN classifier uses different heuristic learning strategies to effectively learn each input spike pattern. It can choose to add a neuron (neuron addition strategy) or skip learning the current spike pattern (skip sample strategy) or update the network parameters (parameter update strategy). The learning algorithm selects the 'neuron addition strategy' when the existing knowledge stored in the network is not enough to approximate the current input spike pattern. The weights and threshold of the newly added neuron are initialized such that it fires precisely for the current input spike pattern. The learning algorithm selects 
the 'skip sample strategy' when the current input spike pattern is similar to previously learned spike patterns. This helps in avoiding over-fitting by preventing the learning algorithm from learning similar samples repeatedly. The 'parameter update strategy' is used by the learning algorithm to fine-tune the existing knowledge stored in the network. The concepts described in this chapter have been published in [82].

The performance of the SRESN classifier has been evaluated on a set of benchmark problem from the UCI machine learning repository [21]. For the purpose of evaluation, the real valued data from the benchmark problems has been encoded into spike patterns using the population coding scheme [17]. To analyze the working of the SRESN classifier and the effect of its algorithm parameters, a study has been conducted using the Ionosphere problem from the UCI machine learning repository. Based on this study, guidelines have been provided to select the different parameters. The performance of the SRESN classifier has also been evaluated for the benchmark problems of Fisher Iris flower classification and Wisconsin breast cancer from the UCI machine learning repository. Performance evaluation results of the SRESN classifier have been compared with that of other existing learning algorithms for spiking neural networks, namely SpikeProp [17], multi-spike neural network [19], multi-spike learning [20] synaptic weight association training [10] and evolving spiking neural network [11].

\subsection{Self-Regulating Evolving Spiking Neural Classi- fier}

In this section, the architecture of the Self-Regulating Evolving Spiking Neural (SRESN) classifier is described, followed by its self-regulating, evolving learning algorithm. The training samples $\left\{\left(\mathbf{x}_{\mathbf{1}}, c_{1}\right), \cdots,\left(\mathbf{x}_{\mathbf{r}}, c_{r}\right), \cdots,\left(\mathbf{x}_{\mathbf{R}}, c_{R}\right)\right\}$ are presented to the network one by one. Here, $\mathbf{x}_{r}=\left[x_{r}^{1}, \cdots, x_{r}^{i}, \cdots, x_{r}^{m}\right] \in \mathbb{R}^{m}$ is the $m$-dimensional input sample and $c_{r} \in\{1, \cdots, N\}$, where $N$ is the total number of classes. Each training sample is encoded into a spike pattern using the population coding scheme [17] and is presented to the network for a fixed duration, called simulation interval $(T)$. In this chapter, $T$ has been set to $3 \mathrm{~ms}$. The goal of the learning algorithm is to estimate the functional relationship between the input features and the class labels such that the decision surface is efficiently 
approximated.

Before describing the architecture of the SRESN classifier, a description of the population coding scheme for converting real valued data into spike patterns is presented.

\subsubsection{Population Coding}

Population coding is the most commonly employed encoding technique for evaluating spiking neural networks on problems with real valued features. Each feature is encoded into spikes using multiple Gaussian receptive fields and each receptive field generates a single spike. Let $x$ be the numerical value of a real valued feature, and $P$ be the number of receptive fields used to encode $x$ into a spike pattern. The center $\left(\mu_{f}\right)$ and width $\left(\sigma_{f}\right)$ of the $f^{t h}$ Gaussian receptive field used for encoding an input feature having range $\left[I_{\min }, I_{\max }\right]$, are initialized as

$$
\begin{aligned}
& \mu_{f}=I_{\text {min }}+\frac{(2 f-3)}{2} \frac{\left(I_{\max }-I_{\min }\right)}{P-2}, \quad f \in\{1, \cdots, P\} \\
& \sigma_{f}=\frac{1}{\gamma} \frac{\left(I_{\max }-I_{\min }\right)}{P-2}, \quad f \in\{1, \cdots, P\}
\end{aligned}
$$

where $\gamma$ is termed as the overlap constant. It determines the extent of overlap between adjacent Gaussian receptive fields by controlling their width.

Based on the center and width of the receptive fields from Equations (3.1) and (3.2) respectively, the activation strength $\left(\phi_{f}\right)$ of the $f^{\text {th }}$ receptive field for $x$ is given as

$$
\phi_{f}=\exp \left(-\frac{\left(x-\mu_{f}\right)^{2}}{2 \sigma_{f}^{2}}\right)
$$

The activation strength of a Gaussian receptive field will always lie in the half closed interval $(0,1]$. Using the activation strength from Equation $(3.3)$, the spike time $\left(t_{f}\right)$ of the $f^{\text {th }}$ receptive field for $x$ is given as

$$
t_{f}=T_{P}\left(1-\phi_{f}\right)
$$

where $T_{P} \in(0, T]$ is the resolution of encoding. It is used to scale the time of spikes generated by the receptive fields from the interval $[0,1]$ to the interval $\left[0, T_{p}\right]$. In this 


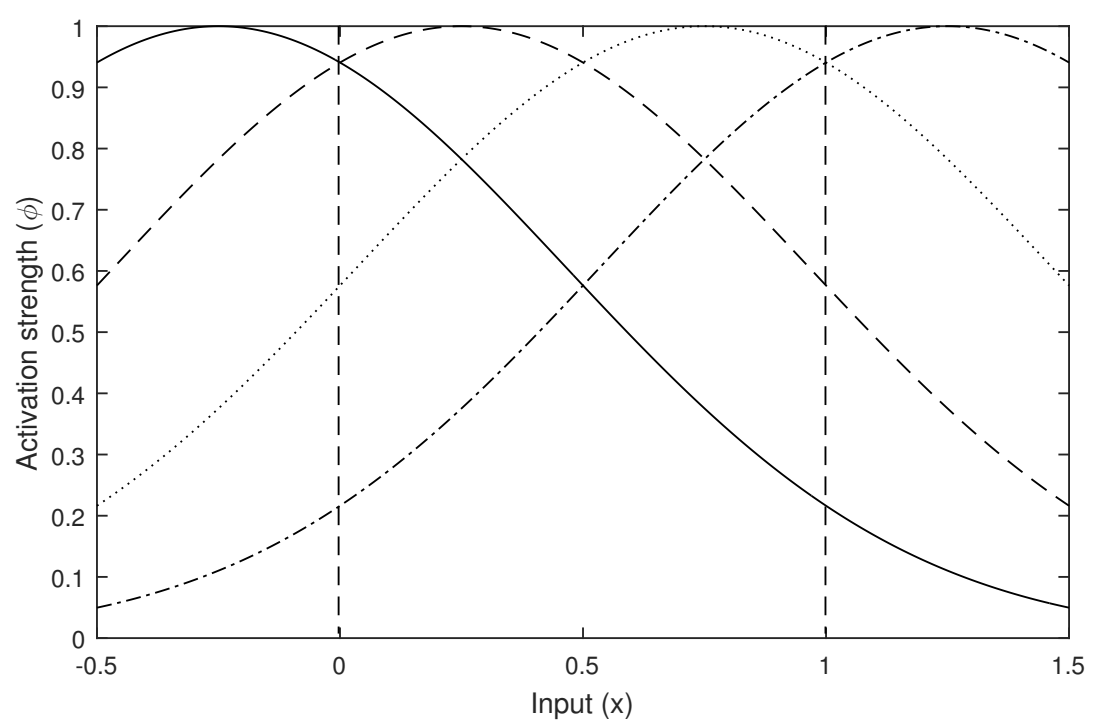

Figure 3.1: An illustrative example of the population coding using four receptive fields for a feature in the range $[0,1]$. The activation strength of different receptive fields is encoded according to different line styles.

work, $T_{P}$ has been fixed at $3 \mathrm{~ms}$.

Figure 3.1 shows an illustrative example where population coding is used to encode a feature in the range $[0,1]$ using four receptive fields. It can be observed from the figure that the centers of two receptive fields are outside the range of the input feature.

\subsubsection{Architecture of the SRESN Classifier}

The proposed SRESN classifier is a two layered network, as shown in Figure 3.2. The nodes in the input layer convert a real valued input into a spike pattern using the population coding scheme [17] without any delays. Suppose, population coding scheme is used with $P$ receptive fields for a single input feature, then the input layer will consist of $m P$ neurons. Each neuron generates a single spike in the simulation interval and the time of spike generated by the $f^{\text {th }}$ neuron encoding the $i^{\text {th }}$ feature is represented as $t_{i f}$. The output layer consists of leaky integrate-and-fire neurons [6] and the postsynaptic potential $\left(v_{j}\right)$ of the $j^{\text {th }}$ output neuron at time $t$ is given as

$$
v_{j}(t)=\sum_{i, f} w_{i j}^{f} \epsilon\left(t-t_{i f}\right)
$$




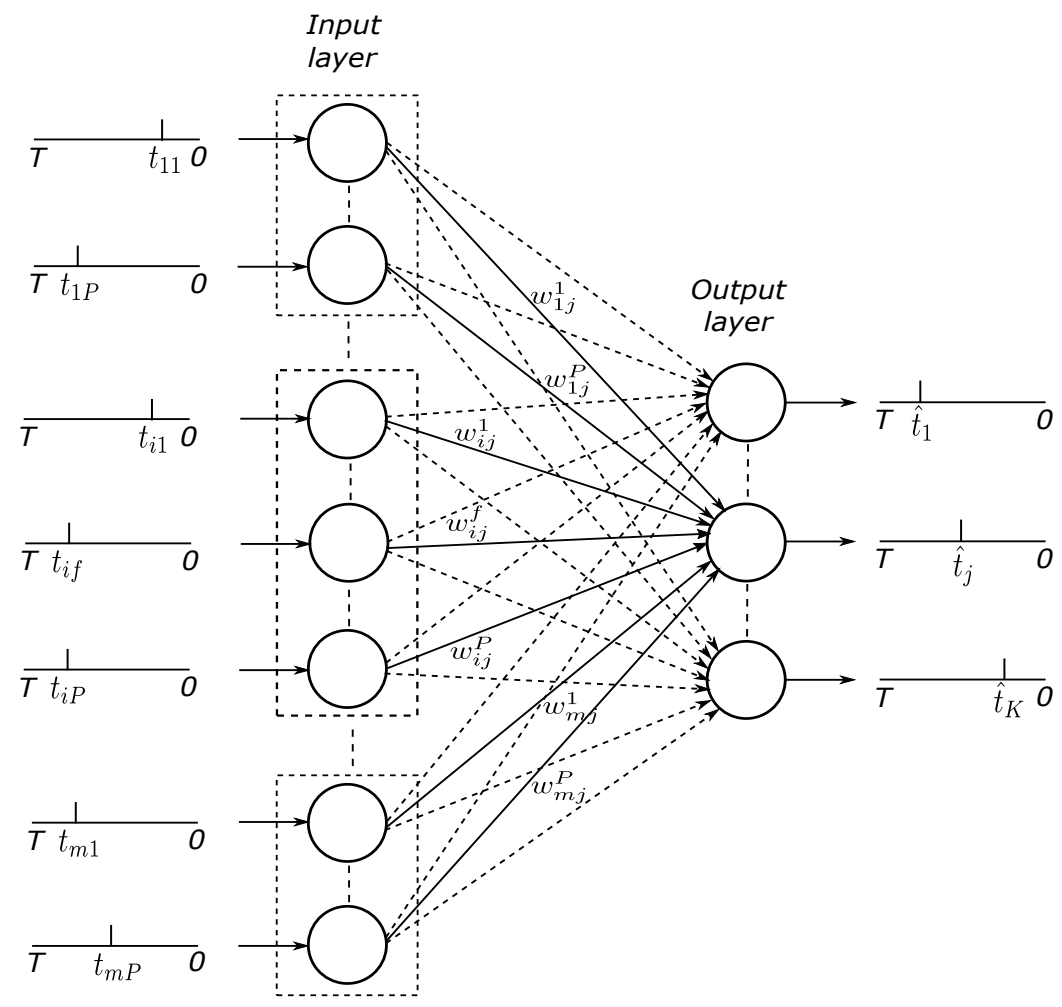

Figure 3.2: The SRESN classifier uses a two layered spiking neural network. The input layer consists of $m P$ neurons for a problem with $m$ features and $P$ being the number of receptive fields used to encode each feature into a spike pattern. Each input neuron generates a single spike in the simulation interval and the time of spike generated by the $f^{t h}$ neuron encoding the $i^{\text {th }}$ feature is denoted by $t_{i f}$.

where $w_{i j}^{f}$ is the weight of the synapse between the $f^{t h}$ neuron encoding the $i^{t h}$ feature and the $j^{\text {th }}$ output neuron. $\epsilon\left(t-t_{i f}\right)$ is the PSP induced, at time $t$, by a spike at $t_{i f}$ on a synapse with weight one. In this work, $\epsilon$ is modeled using the spike response function [17], given as

$$
\epsilon(s)=\frac{s}{\tau} \exp \left(1-\frac{s}{\tau}\right)
$$

where $\tau$ is the time constant for the spiking neuron.

The PSP of an output neuron is reset to zero immediately after the neuron generates a spike. Suppose $\theta_{j}$ represents the threshold of the $j^{\text {th }}$ output neuron, then the time of 
the first spike $\left(\hat{t}_{j}\right)$ generated by the $j^{\text {th }}$ output neuron is given as

$$
\begin{aligned}
& \hat{t}_{j}=\underset{t}{\arg }\left(v_{j}(t)>\theta_{j}\right) \\
& \lim _{t \rightarrow \hat{t}_{j}^{+}} v_{j}(t)=0
\end{aligned}
$$

It may be observed that the output neurons can generate more than one spike, but the learning algorithm relies only on the first spike generated by any output neuron to determine the predicted class. The predicted class for a given input sample is determined based on the class association of the output neuron that fires first. Suppose, $l$ represents the index of the output neuron that spiked first (neuron having minimum latency) and $c_{l}$ is the class associated with that neuron, then, $c_{l}$ is the predicted class $\left(\hat{c}_{r}\right)$ for the current input sample $\left(\mathbf{x}_{r}\right)$.

Since the predicted class is determined based on the time of the first spike generated by any output neuron, the desired time of the first spike (target firing time) for that output neurons is defined as follows. The target firing time for the output neuron from the same class as the current sample is represented by a spike at a fixed instant, $T_{0} \in[0, T]$ whereas other class neurons should not generate a spike within the simulation interval. Thus, the desired spike time $\left(t_{j}\right)$ of the $j^{\text {th }}$ output neuron is given as

$$
t_{j}= \begin{cases}T_{0} & c_{j}=c_{r} \\ T+\delta & c_{j} \neq c_{r}\end{cases}
$$

where $\delta$ is a small positive number, such that a target firing time of $(T+\delta)$ implies that the particular neuron does not spike during the simulation interval $([0, T])$.

\subsubsection{Learning Algorithm for the SRESN Classifier}

The learning algorithm for the SRESN classifier is a self-regulating, evolving learning algorithm which starts with no output neurons and evolves/adapts the network. As a sample is presented to the network, the learning algorithm measures the knowledge present in the current sample with respect to the knowledge stored in the network. Based on this, the learning algorithm selects to either skip learning the sample (skip sample strategy) or add 
a new neuron (neuron addition strategy) or update the network parameters (parameter update strategy) such that the knowledge contained in the sample is well generalized. Next, we will describe these learning strategies in detail.

Without loss of generality, let us assume that the network has evolved to $K$ output neurons. Also, $\hat{t}_{C C}$ and $\hat{t}_{M C}$ represent the spike times for a correct class neuron and a different class neuron having minimum spike latency, respectively. Here, $C C$ represent the output neuron from the correct class that fires first, given as

$$
C C=\underset{j, c_{j}=c_{r}}{\operatorname{argmin}} \hat{t}_{j}
$$

$M C$ represents the neuron from any other class that fires first, given as

$$
M C=\underset{j, c_{j} \neq c_{r}}{\operatorname{argmin}} \hat{t}_{j}
$$

- Skip sample strategy: If the knowledge in the current sample is similar to the knowledge stored in the network and difference in the spike times of interclass neurons is significantly higher then the sample is skipped from being learned. This strategy prevents the network from over training on similar samples, thereby resulting in higher generalization and also reduced computational effort.

To illustrate the working of skip sample strategy, let us consider a scenario as given in Figure 3.3. The figure shows the firing time of two neurons in the simulation interval along with the target firing time $\left(T_{0}\right)$. It could be seen that $t_{C C}$ is closer to the target firing time, which indicates that the information present in the current

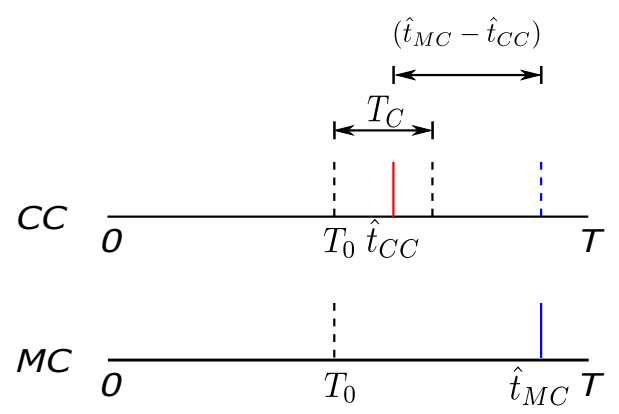

Figure 3.3: An illustrative example of the 'skip sample strategy' 
sample is already present in the network. Further, it could be seen that $C C$ fires significantly earlier than $M C\left(t_{C C}<t_{M C}\right)$ implying that the current sample is well separated from other class neurons. Hence, the knowledge contained in this sample is well generalized, and it could be skipped from the learning process. The criterion for skip sample strategy is given as,

$$
\text { If }\left(c_{r}=\hat{c}_{r}\right) \&\left(\left(\hat{t}_{C C}-T_{0}\right)<T_{C}\right) \&\left(\left(\hat{t}_{M C}-\hat{t}_{C C}\right)>T_{C}\right)
$$

Then skip learning the sample

where $T_{C} \in\left[0,\left(T-T_{0}\right)\right]$ is termed as the confidence threshold. If $T_{C}$ is set closer to zero, then the learning algorithm ensures that the correct class output neuron fires closer to the target firing time $\left(T_{0}\right)$ without focusing on maintaining a high interclass separation. This may result in poor generalization performance due to lower interclass separation. On the other hand, if $T_{C}$ is set to a higher value, the learning algorithm maintains a high interclass separation without emphasizing a smaller separation between the target firing time and $\hat{t}_{C C}$. This may result in loss of knowledge already stored in the network. In this work, $T_{C}$ is fixed at $0.6 \mathrm{~ms}$.

- Neuron addition strategy: The learning algorithm adds a neuron to the network in two distinct scenarios. Firstly, a new neuron is added to the network when the current sample contains a significant amount of information (neuron addition to handle novelty). Secondly, a new neuron is added to the network when the current sample has considerable overlap with another class neuron (neuron addition to handle overlap). Next, the two scenarios will be described with the help of illustrative examples.

(a) Neuron addition to handle novelty: Figure 3.4a and 3.4b show the illustrative examples of this strategy. It could be observed from Figure 3.4a that the current sample is correctly classified as $C C$ fires before $M C$. But, $C C$ fires very late $\left(t_{C C}>T_{C C}\right)$ in the simulation interval, which indicates that the knowledge in the network is not enough to approximate the current sample. Similarly, it can be observed from Figure 3.4b, that a different class neuron $(M C)$ fires very late $\left(t_{C C}>T_{M C}\right)$ in the simulation interval, which implies that the current sample can 
not be approximated by the existing knowledge in the network. Hence, a new neuron is added to the network to capture the knowledge present in the input sample in both these scenarios. The neuron addition criterion for these scenarios is given as:

$$
\text { If }\left(\left(c_{r}=\hat{c}_{r}\right) \&\left(\hat{t}_{C C}>T_{C C}\right)\right) \text { or }\left(\left(c_{r} \neq \hat{c}_{r}\right) \&\left(\hat{t}_{M C}>T_{M C}\right)\right)
$$

Then add a neuron

where $T_{C C} \in\left[T_{0}, T\right]$ and $T_{M C} \in\left[T_{0}, T\right]$ are the neuron addition thresholds in case a sample is correctly classified and misclassified, respectively. These thresholds control the number of neurons added by the SRESN classifier to the network. A higher value for the neuron addition thresholds results in addition of fewer neurons to the network. On the other hand, a lower value results in the addition of

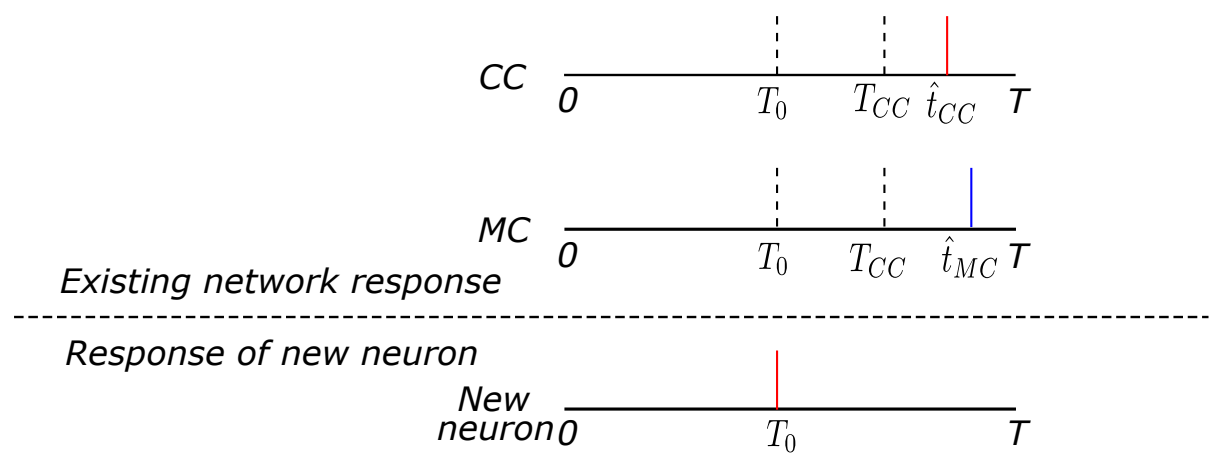

(a) An illustrative example of the 'neuron addition to handle novelty' in case of correct classification

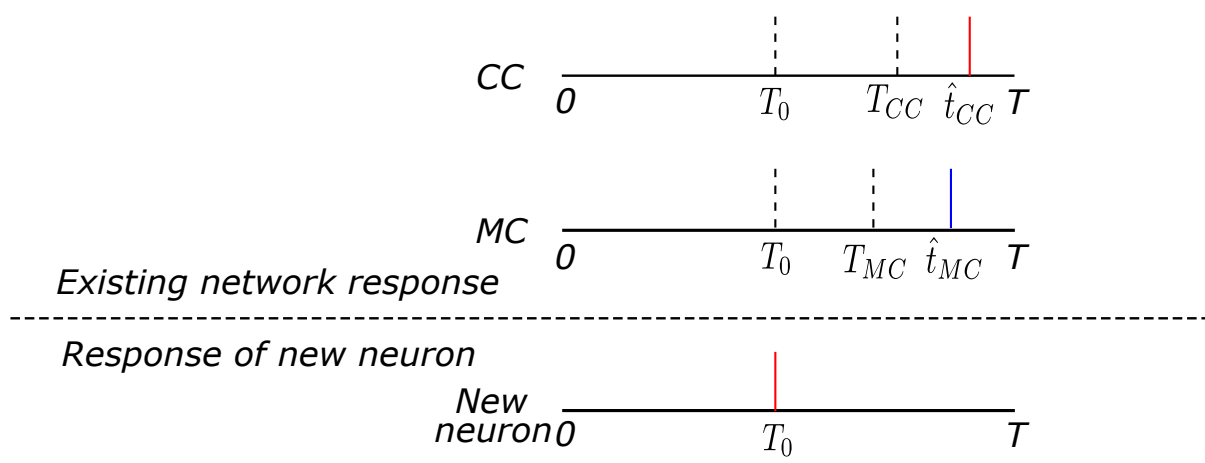

(b) An illustrative example of the 'neuron addition to handle novelty' in case of misclassification

Figure 3.4: Illustrative examples of adding a neuron to handle novelty 
too many neurons. These thresholds affect the accuracy with which the SRESN classifier approximates the functional relationship between input samples and the corresponding class labels.

The weights for the neuron are initialized using a modified rank order learning scheme. Rank order learning is a well-known technique for weight initialization in an online learning framework [12]. Rank Order Learning (ROL) initializes weights based on the order of spikes. This may result in non-contributing weights, for problems with a large number of features. To overcome these issues, SRESN classifier employs ROL in a feature-wise manner. This helps in improving the discriminability of the input samples. Using the modified ROL, the rank of the $f^{\text {th }}$ input neuron encoding the $i^{\text {th }}$ feature is given as

$$
\operatorname{rank}(i, f)=\sum_{b=1, b \neq f}^{P} F_{\text {rank }}(i, f, b)
$$

where $F_{\text {rank }}$ is termed as the ranking function, given as

$$
F_{\text {rank }}(i, f, b)= \begin{cases}0 & t_{i f} \leq t_{i b} \\ 1 & t_{i f}>t_{i b}\end{cases}
$$

Based on the rank of a given input neuron, the weights of the newly added neuron $\left(\mathbf{w}_{(K+1)}=\left[w_{1(K+1)}^{1}, \cdots, w_{i(K+1)}^{f}, \cdots, w_{m(K+1)}^{P}\right]\right)$ are initialized as

$$
w_{i(K+1)}^{f}=\lambda^{\operatorname{rank}(i, f)}, \quad i \in\{1, \cdots, m\} ; f \in\{1, \cdots, P\}
$$

where $\lambda$ is termed as the modulation factor and is set in the interval $[0,1]$. It controls the rate at which the weights of synapses with subsequent spikes decrease using ROL. If $\lambda$ is set closer to zero, then there is a higher difference in the weights of two synapses with a lower difference in the spike times of the corresponding input neurons. If $\lambda$ is set closer to one, then there is a smaller difference in the weights of two synapses with a higher difference in the spike times of the corresponding input neurons. In both cases, the information present in current input sample is 


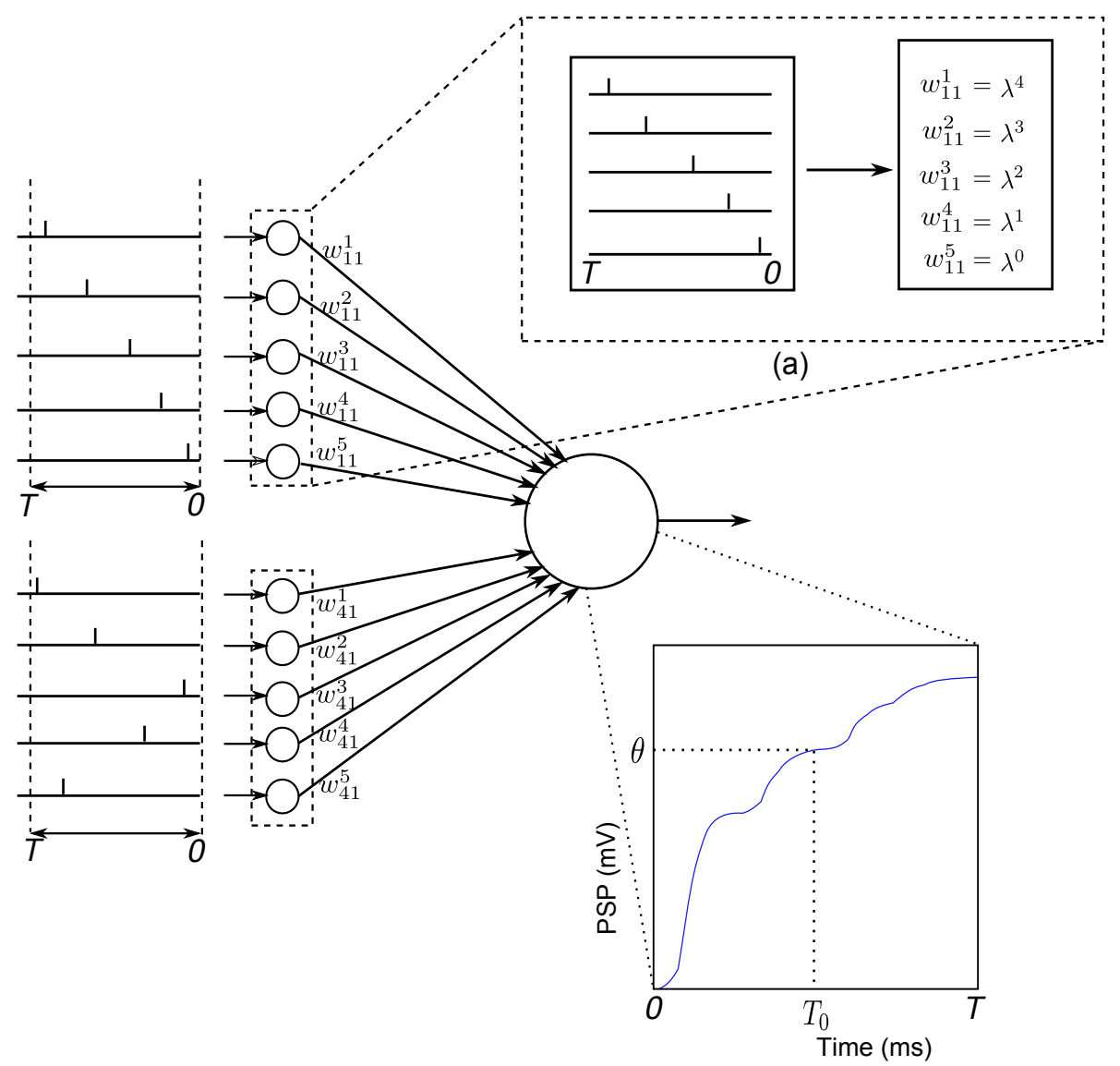

(b)

Figure 3.5: An illustrative example of the procedure for initializing weights and threshold of a newly added neuron using a problem with two features, each encoded using five receptive fields. (a) Initial value of weights, determined using modified rank order learning, for the input neurons encoding the first feature, (b) PSP of the newly added neuron in the interval $[0, T]$ with initialized weights. The PSP of the neuron at $T_{0}$ is set as the threshold for the neuron. 
not properly stored in the network. As a result, the modulation factor $(\lambda)$ has to be initialized properly and its value should be set closer to one.

Using the initialized weights, the threshold $\left(\theta_{(K+1)}\right)$ for the newly added neuron is initialized as the PSP of the neuron at the target firing time $\left(T_{0}\right)$, given as

$$
\theta_{(K+1)}=\sum_{i, f} w_{i(K+1)}^{f} \epsilon\left(T_{0}-t_{i f}\right)
$$

Here, $T_{0}$ determines the amount of information in a given input spike pattern that is stored by the network and is used to classify subsequently presented spike patterns. If $T_{0}$ is set to a value closer to zero, it may impede the ability of the network to properly learn from the input spike patterns. If $T_{0}$ is set to a value closer to $T$, the network will take a long time to determine the predicted class for a given spike pattern. Based on this, the value of $T_{0}$ is set to $T / 2$ as a tradeoff between the learning capability of the network and the speed of the network in determining the predicted class.

The initialization of neuron weights and threshold using Equation 3.16 and 3.17, respectively, ensures that the newly added neuron spikes precisely at the target firing time $\left(T_{0}\right)$ for the current input sample. Figure 3.5 illustrates the procedure for initializing weights and threshold for the newly added neuron using an example problem with two features, each encoded using five input neurons. Figure 3.5a shows the initial value of weights, determined using modified ROL, for the input neurons encoding the first feature. It can be observed from the figure that the input neuron that spikes first will have the highest weight and the input neuron that spikes last will have the lowest weight. Figure 3.5b shows the variation in PSP of the newly added neuron for the weights initialized using modified ROL. The PSP of the neuron at $T_{0}$ is set as the threshold for the neuron, thereby ensuring that the particular neuron fires precisely at $T_{0}$ for the current input sample.

(b) Neuron addition to handle overlap: Figure 3.6 shows an illustrative example of this strategy. It could be observed from the figure that the predicted class label for the current input sample is, although a neuron in the network is firing close to the target firing time. In this situation, the learning algorithm adds a neuron to the 

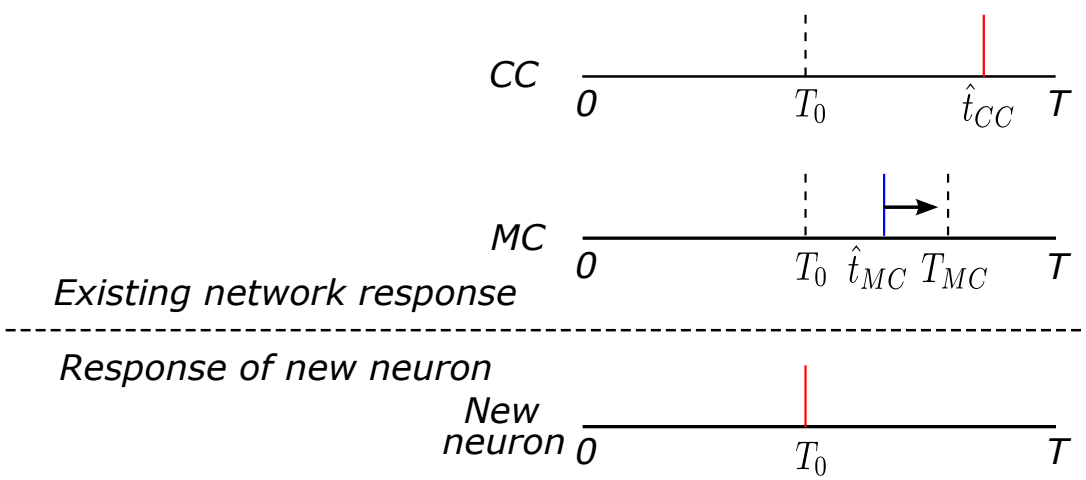

Figure 3.6: An illustrative example of the 'neuron addition to handle overlap'

network to capture the knowledge present in the current sample and also updates the synaptic weights for the different class neuron to minimize misclassification. The weights of the different class neuron are updated such that it fires late for the current input sample (in the direction of the arrow in Figure 3.6). The criterion for selecting this strategy is given as

$$
\text { If }\left(c_{r} \neq \hat{c}_{r}\right) \&\left(\hat{t}_{M C}<T_{M C}\right) \&\left(\left(\hat{t}_{C C}-\hat{t}_{M C}\right)>T_{C} / 2\right)
$$

Then add a neuron and update the neuron MC

The weights and threshold for the new neuron are initialized using Equation 3.16 and 3.17, respectively. While updating the weights of the neuron $M C$, the learning algorithm does not update the weights for all the input neurons. The weights for input neurons with a non-zero PSP at $t_{M C}$ are alone updated. Further, the synapses with higher weights i.e. the input neurons contributing significantly towards PSP of the neuron $M C$ are updated. Thus, the set $\left(\psi_{M C}\right)$ of synapses whose weights are updated is given as

$$
\begin{aligned}
\psi_{M C}=\{(p, q) \mid p \in\{1, \cdots, m\}, q \in\{1, \cdots, P\} \\
\\
\left.\left(\epsilon\left(\hat{t}_{M C}-t_{p q}\right)>0\right),\left(w_{p(M C)}^{q}>\lambda^{\operatorname{rank}(p, q)}\right)\right\}
\end{aligned}
$$

where $(p, q)$ represents the synapse between the $q^{t h}$ input neuron encoding the $p^{t h}$ feature and the output neuron $M C$. The change in the weight $\left(\Delta w_{p(M C)}^{q}\right)$ of the synapse between the $q^{\text {th }}$ input neuron encoding the $p^{\text {th }}$ feature and the output 
neuron $M C$ is given as

$$
\Delta w_{p(M C)}^{q}=\eta\left(\lambda^{\operatorname{rank}(p, q)}-w_{p(M C)}^{q}\right), \quad \forall(p, q) \in \psi_{M C}
$$

where $\eta$ is the learning rate.

- Parameter update strategy: The parameters of the network are updated when there is some knowledge (refining the knowledge is required) in the sample, but not enough to add a neuron. In this work, if none of the above strategies of skip sample or neuron addition are triggered, then the weights of the neurons are updated. Similar to the neuron addition strategy, there are two scenarios in parameter update strategy: (a) parameter update to handle novelty and (b) parameter update to handle overlap. If the interclass separation is lesser than $T_{C} / 2$, i.e. $\left|t_{M C}-t_{C C}\right|<$ $T_{C} / 2$, the weights for both the neurons $C C$ and $M C$ are updated to handle overlap between classes. Otherwise, the learning algorithm updates only the weights of the neuron $C C$. Both the scenarios are described below with illustrative examples.

(a) Parameter update to handle novelty: The learning algorithm employs this strategy when the class of a sample is correctly predicted with high interclass margin, but the neuron $C C$ does not fire closer to the target firing time $\left(T_{0}\right)$. Figure 3.7 shows an example of the parameter update strategy for handling novelty. It can be observed from the figure that the neuron $C C$ fires before $T_{C C}$ but its spike time is not close to $T_{0}$ which implies that the network does not have sufficient knowledge to accurately approximate the information present in the current input sample. In this case, the learning algorithm updates the weights of the neuron $C C$ to ensure

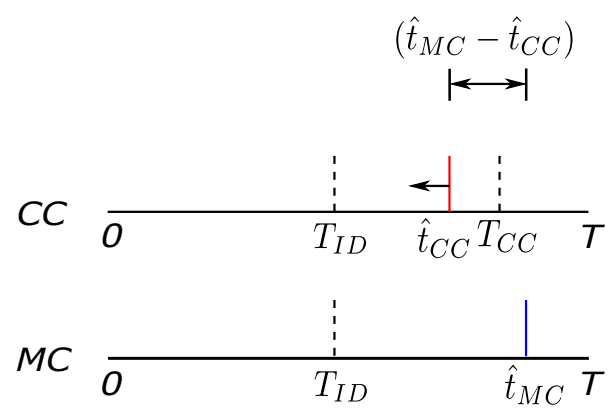

Figure 3.7: An illustrative example of the 'parameter update strategy to handle novelty' 
that the neuron $C C$ fires closer to the target firing time (shown by the arrow in the Figure 3.7).

For this strategy as well, the learning algorithm does not update the weights for all the synapses between input neurons and the neuron $C C$. The learning algorithm updates the weights for the input neurons with, non-zero contribution to PSP at $t_{C C}$. Further, the synapses with lower weights are updated to ensure that the previously learnt information is retained. Thus, the set $\left(\psi_{C C}\right)$ of synapses whose weights are updated is given as

$$
\begin{array}{r}
\psi_{C C}=\{(p, q) \mid p \in\{1, \cdots, m\}, q \in\{1, \cdots, P\} \\
\left.\left(\epsilon\left(\hat{t}_{C C}-t_{p q}\right)>0\right),\left(w_{p(C C)}^{q}<\lambda^{\operatorname{rank}(p, q)}\right)\right\}
\end{array}
$$

The change in the weight $\left(\Delta w_{p(C C)}^{q}\right)$ of the synapse between the $q^{t h}$ input neuron encoding the $p^{\text {th }}$ feature and the output neuron $C C$ is given as

$$
\Delta w_{p(C C)}^{q}=\eta\left(\lambda^{\operatorname{rank}(p, q)}-w_{p(C C)}^{q}\right), \quad \forall(p, q) \in \psi_{C C}
$$

(b) Parameter update to handle overlap: The learning algorithm chooses this strategy when a sample is very close to the decision boundary. Figure 3.8a and 3.8b show two examples where the learning algorithm chooses this strategy to update the network parameters. It can be observed from the Figure 3.8a, that the sample is correctly classified but the spike time of the neuron $C C$ is very close to the spike

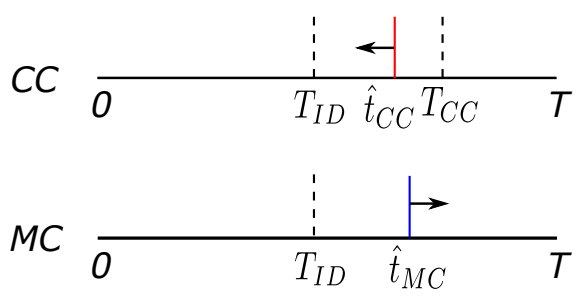

(a) An illustrative example of the 'parameter update strategy to handle overlap' in case of correct classification
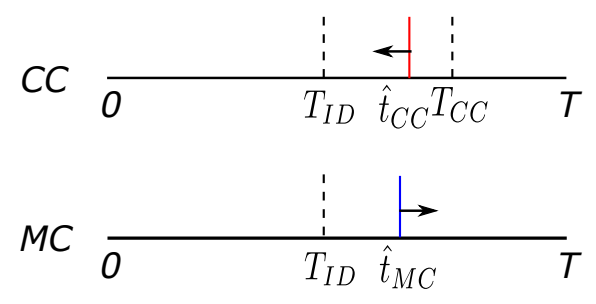

(b) An illustrative example of the 'parameter update strategy to handle overlap' in case of incorrect classification

Figure 3.8: Illustrative examples of the 'parameter update strategy to handle overlap' 
time of the neuron $M C$. Similarly, Figure $3.8 \mathrm{~b}$ shows an example where the sample is misclassified, but the spike times of the neurons $C C$ and $M C$ are very close to one another. In both the situation, the learning algorithm updates the weights for both the neurons, $C C$ and $M C$, so as to shift their spike times in the directions indicated by the arrow (in the Figure 3.8a and 3.8b).

The weights for the neurons $C C$ and $M C$ are updated using the Equations (3.22) and (3.20) respectively.

These three strategies help the SRESN classifier in self-regulating its learning process to learn each sample appropriately. They are repeated for multiple epochs until the desired level of accuracy is achieved. A single epoch of the proposed SRESN classifier has been summarized in Figure 3.9 using a flowchart format.

\subsection{Working of the SRESN Classifier Using the Iono- sphere Problem}

In this section, the Ionosphere problem from the UCI machine learning repository is used to study the effects of different parameters on the performance of the SRESN classifier. For this purpose, a simulation interval of $3 \mathrm{~ms}$ is used and the target firing time is fixed at $1.5 \mathrm{~ms}$. The performance of the SRESN classifier is computed using the overall classification accuracy which is equal to the percentage of samples that are correctly classified. Based on this study, several guidelines are also provided for setting the different parameters. The algorithm parameters that affect the performance of the learning algorithm are modulation factor $(\lambda)$, neuron addition threshold for correct classification $\left(T_{C C}\right)$ and neuron addition threshold for misclassification $\left(T_{M C}\right)$. The Ionosphere problem is chosen for this study as it contains a large number of features and requires non-linear separability. The observations from this section are also used to deduce a suitable range for initializing the value of different algorithm parameters.

The problem of Ionosphere consists of radar data collected from a phased array of 16 high frequency antennas. The target of the transmitted signals is the free electrons in the atmosphere. Each sample consists of 34 attributes and could be classified as a good 


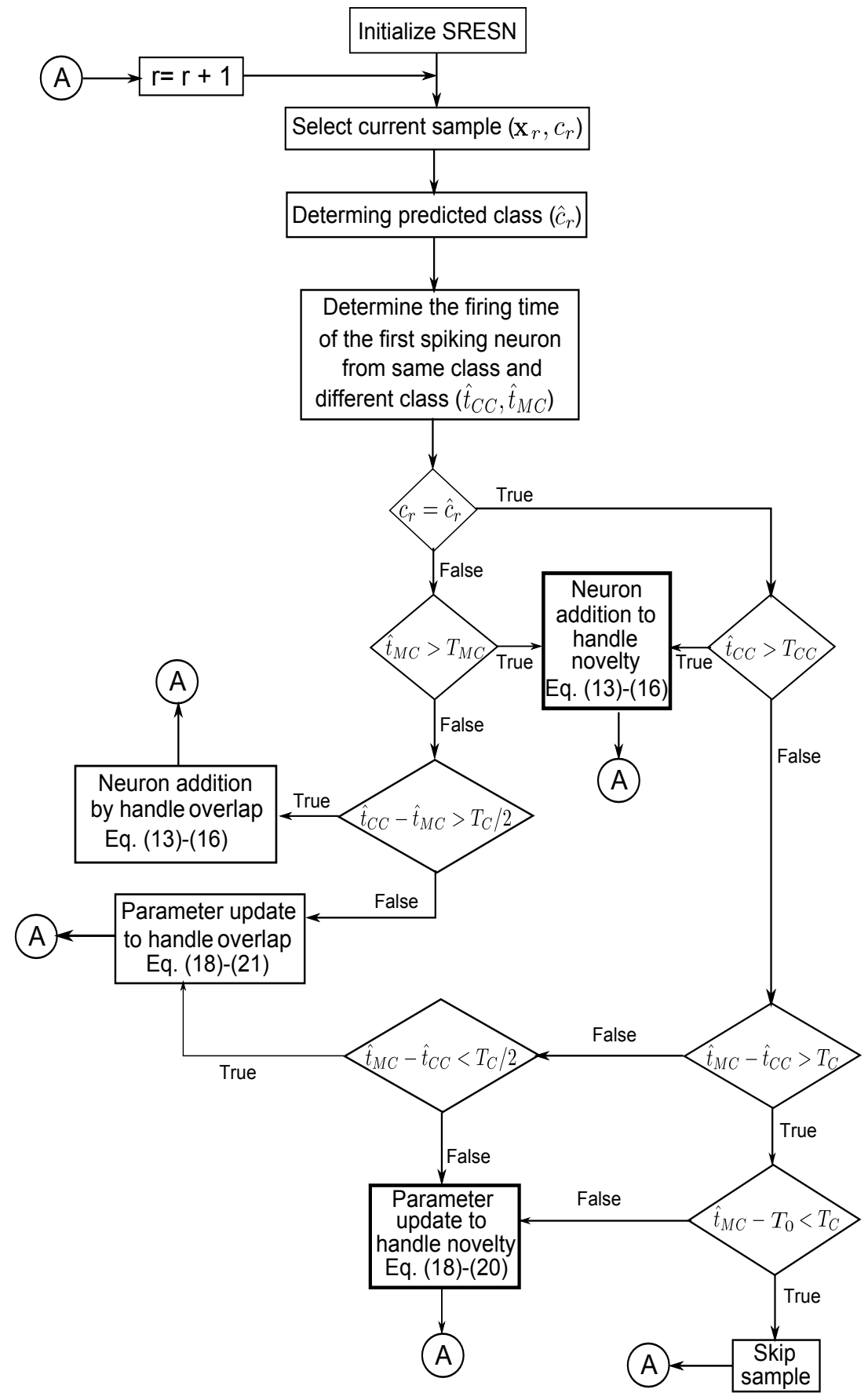

Figure 3.9: A flow chart depicting a single epoch of the learning algorithm for the SRESN classifier. 


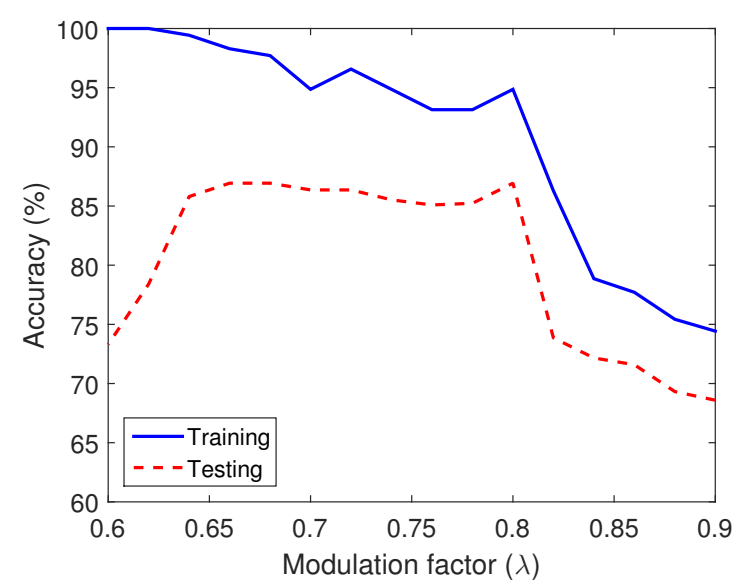

(a)

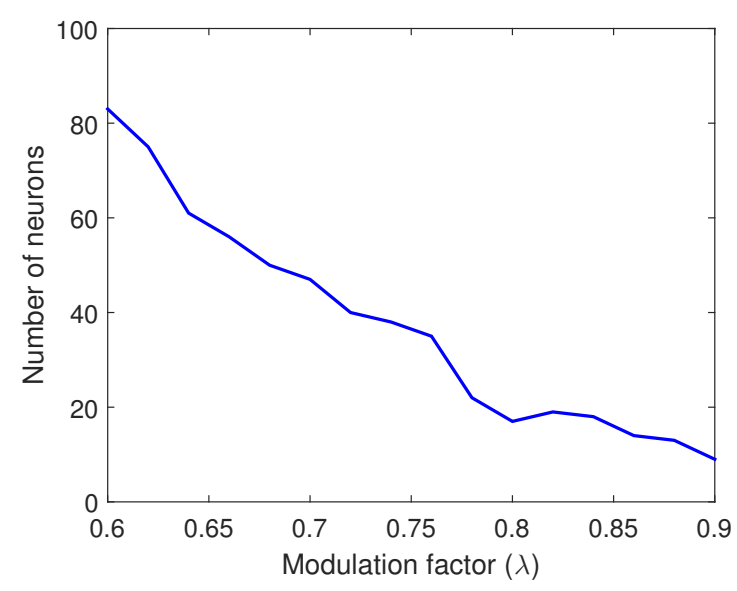

(b)

Figure 3.10: Effect of modulation factor $(\lambda)$ on the (a) Overall training and testing accuracy, (b) Number of neurons added by the SRESN classifier for the Ionosphere problem

or bad sample depending on whether it contains any information about the structure of the ionosphere or not. The problem consists of a total of 351 samples, 175 of which are used for training and the rest are used for testing. Next, the Ionosphere problem will be used to illustrate the impact of different algorithm parameters on the performance of the SRESN classifier.

- Modulation factor $(\lambda)$ : Modulation factor determines the initial value of weights estimated using rank order learning. The weights of the synapses are initialized such that the weight of the input neuron that spikes first will be highest and for subsequent spikes, the weights decrease exponentially. If the value of $\lambda$ is set closer to zero, then the weights quickly decay to zero. If its value is set closer to one, then there is a smaller difference in the weights of the different synapses. In both cases, the information present the sample is not properly learned by the network. Figure 3.10a shows the effect of modulation factor on the training and testing accuracy of the SRESN classifier, when $\lambda$ is varied in the interval $[0.6,0.9]$. It can be observed from the figure, that the testing accuracy shows a small variation when $\lambda$ is varied in the interval $[0.65,0.8]$ and outside this range testing accuracy decreases rapidly. Figure $3.10 \mathrm{~b}$ shows the effect of modulation factor on the number of output neurons added by the SRESN classifier for different values of $\lambda$ in the interval $[0.6,0.9]$. It can be observed from the figure that for smaller values of $\lambda$, the learning algorithm 


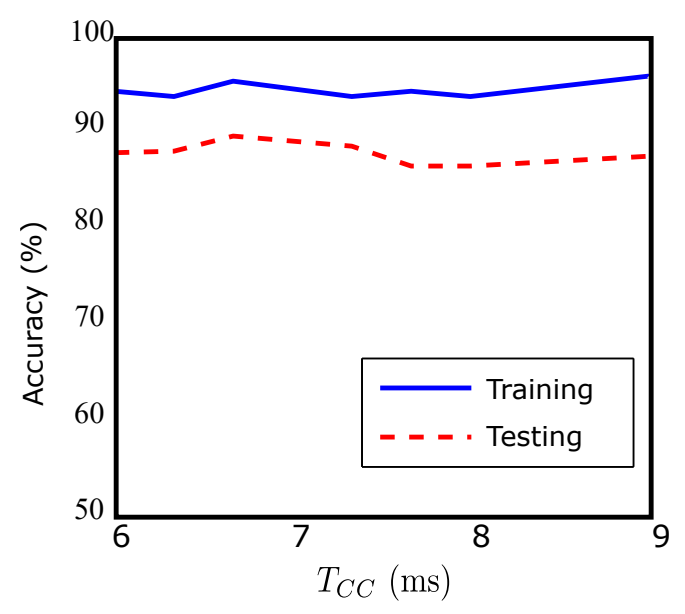

(a)

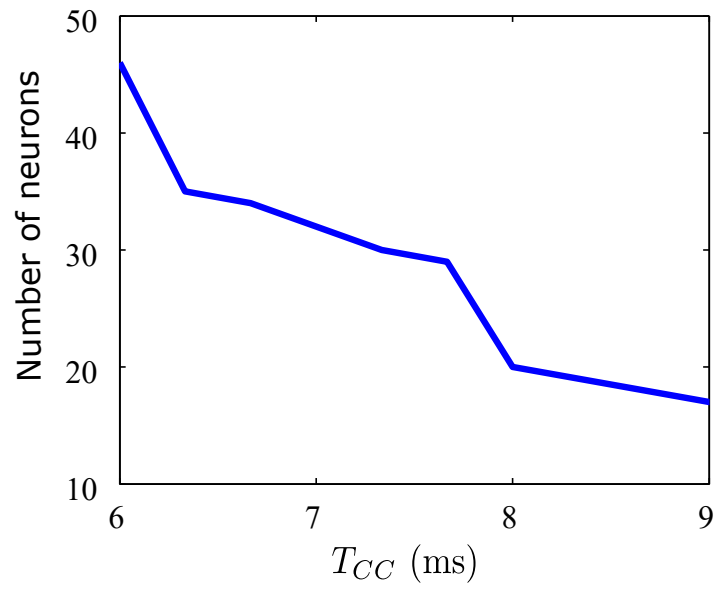

(b)

Figure 3.11: Effect of neuron addition threshold in case of correct classification $\left(T_{C C}\right)$ on (a) Overall training and testing accuracy, (b) Number of neurons added by the SRESN classifier for the Ionosphere problem

adds a large number of neurons to the network. For smaller values of $\lambda$, the weights for the input neurons quickly decay to zero; as a result the information present in the input sample is not stored in the network properly. Consequently, the learning algorithm requires more neurons to approximate the information present in the input samples. Based on these observations, a suitable range for setting $\lambda$ is the interval $[0.65,0.8]$. In this work, $\lambda$ is set to 0.8 so as to learn using a compact network architecture.

- Neuron addition threshold for correct classification $\left(T_{C C}\right): T_{C C}$ can be set to a value in the interval $\left[T_{0}, T\right]$. Since, $T_{0}$ is set to $1.5 \mathrm{~ms}$ and $T$ is set to $3 \mathrm{~ms}$, the performance of the SRESN classifier has been evaluated for values of $T_{C C}$ in the interval $[1.6,3] \mathrm{ms}$. If $T_{C C}$ is set to a higher value, it causes fewer neurons to be added to the network and a low value of $T_{C C}$ leads to the addition of a large number of neurons. Both these cases affect the generalization ability of the SRESN classifier. Figure 3.11a shows the effect of $T_{C C}$ on the overall training and testing accuracy of the SRESN classifier. Figure 3.11b shows the impact of $T_{C C}$ on the number of neurons added by the learning algorithm for the SRESN classifier. It can be seen from the figures that there is a small variation in the overall training 
and testing accuracy for different values of $T_{C C}$ in the interval $[1.5,3] \mathrm{ms}$, but more neurons are added to the network for smaller values of $T_{C C}$. A suitable range for setting $T_{C C}$ is the interval $[2.1,2.7] \mathrm{ms}$ to achieve good performance using fewer neurons.

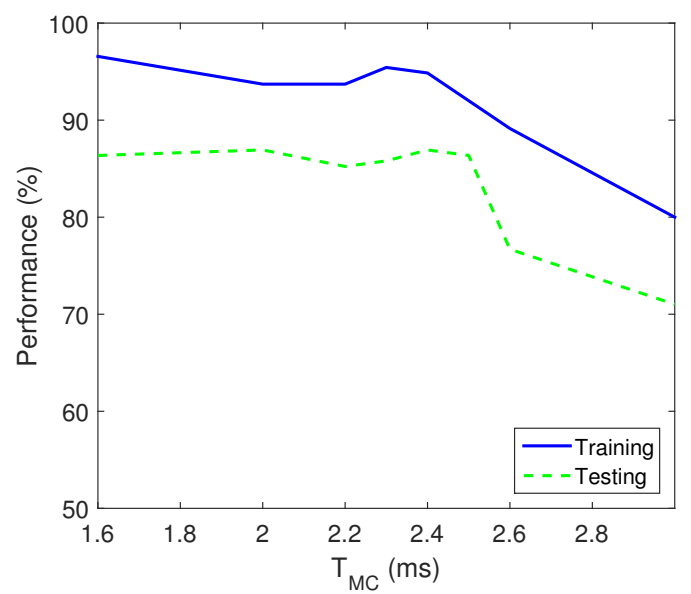

(a)

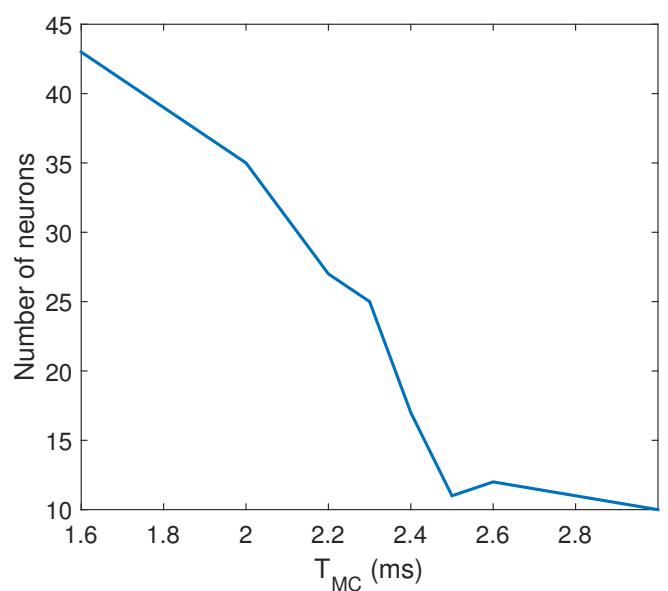

(b)

Figure 3.12: Effect of neuron addition threshold in case of misclassification on (a) Overall training and testing accuracy, (b) Number of neurons added by the SRESN classifier for the Ionosphere problem

- Neuron addition threshold for misclassification $\left(T_{M C}\right)$ : Similar to $T_{C C}$, the performance of the learning algorithm is studied for the values of $T_{M C}$ in the interval $[1.6,3] \mathrm{ms}$. Figure 3.12a shows the effect of $T_{M C}$ on the overall training and testing accuracy of the SRESN classifier. Figure 3.12b shows the impact of $T_{M C}$ on the number of neurons added by the learning algorithm for the SRESN classifier. The effect of $T_{M C}$ on the learning algorithm is similar to that of $T_{C C}$. A higher value for $T_{M C}$ leads to the addition of fewer neurons to the network and a smaller value results in the addition of higher number of neurons. This can also be observed clearly from Figure 3.12b. From Figure 3.12a, it can be observed that there is a small variation in the accuracy as $T_{M C}$ is varied from 1.8 to $2.4 \mathrm{~ms}$. But when $T_{M C}$ is set to a value in the range 2.4 to $3 \mathrm{~ms}$, the accuracy deteriorates. The lower accuracy might be due to the lower number of neurons used by the SRESN classifier for a higher value of $T_{M C}$. Hence, a suitable interval for setting $T_{M C}$ is $[1.8,2.4] \mathrm{ms}$. 
The suitable intervals for choosing $T_{C C}$ and $T_{M C}$ are $[2.1,2.7] \mathrm{ms}$ and $[1.8,2.4] \mathrm{ms}$ respectively. For simple problems, with no overlap between classes, $T_{C C}$ and $T_{M C}$ should be chosen closer to 2.7 and $2.4 \mathrm{~ms}$, respectively as acceptable accuracy can be achieved with fewer neurons. On the other hand, for difficult problems, with significant overlap between the classes, the values of $T_{C C}$ and $T_{M C}$ should be set closer to 2.1 and 1.8 $m s$, respectively, as more neurons are needed for an accurate representation of the data. Next, the performance of the SRESN classifier is evaluated on two benchmark problems from the UCI machine learning repository and the results of performance evaluation are compared with that of the other learning algorithms for SNNs.

\subsection{Performance Comparison of the SRESN Classi- fier}

In this section, the performance of the proposed SRESN classifier is evaluated on benchmark problems from the UCI machine learning repository [21]. The results of performance evaluation of the SRESN classifier are compared with that of the other existing batch learning algorithms for spiking neural networks, namely SpikeProp [17], Multi-Spike Neural Network (MuSpiNN) [19], Multi-spike learning [20] Synaptic Weight Association Training (SWAT) [10] and evolving Spiking Neural Network (eSNN) [11]. The results of performance evaluation for Spikprop, MuSpiNN, Multi-spike learning, SWAT and eSNN have been reproduced from [17], [19], [20], [10] and [61], respectively. The results for SpikeProp have been generated using two-fold cross validation whereas the results for SWAT have been generated using five-fold cross validation. The results for Multi-spike learning have been averaged over a large number of experiments. For MuSpiNN and eSNN, the number of folds used have not been reported in the paper. The results for the SRESN classifier are generated using ten random trials. The mean and standard deviation for ten random trials are reported in this section.

The performance of the different algorithms are compared based on three metrics: overall testing accuracy (ratio of correctly classified samples to the total number of samples), the number of network parameters and the number of epochs required for an 
algorithm for convergence. The number of network parameters is calculated as,

$$
\text { No. of network parameters }=m P * n_{d(h)} * K+K * n_{d(o)} * C
$$

where $n_{d(h))}$ and $n_{d(o)}$ are the number of delays used in the hidden layer and output layer respectively. $C$ is the number of neurons in the output layer. $n_{d(h))}$ and $n_{d(o)}$ are set to one for the SRESN classifier as it employs population coding without any delays. $C$ is equal to zero for the SRESN classifier as it employs a two layered SNN.

The real valued data from the benchmark problems is encoded into spike patterns using population coding scheme for all the learning algorithms except, SWAT. In the case of SWAT, cosine coding [83] is used to encode data from the benchmark problems into spike patterns. For the SRESN classifier, a simulation interval of $3 \mathrm{~ms}$ is used and the target firing time is fixed at $1.5 \mathrm{~ms}$. The training phase for the SRESN classifier is stopped when either the desired training accuracy is achieved or number of epochs exceeds 1000 .

All the simulations in this study are conducted on a machine with 12 logical cores, 16GB memory and CPU speed of $3.2 \mathrm{GHz}$ using MATLAB 2013b running on Windows operating system.

\subsubsection{Iris Flower Classification Problem}

The Iris flower classification is a three class problem, and each class has fifty samples with four features in each sample. During this study, ten samples are randomly chosen from each class. These samples are used for training and the rest of the samples are used for testing. For the SRESN classifier, the value of $T_{C C}$ and $T_{M C}$ are set as $2.1 \mathrm{~ms}$ and $1.8 \mathrm{~ms}$, respectively.

Before discussing the results of the performance evaluation, the process for determining the predicted class from the response of output neurons will be described. Figure 3.13 shows the response of the network for five random testing samples from the Iris data set after the training phase is over. Each row in the figure represents the response $\left(\hat{y}^{1}, \cdots, \hat{y}^{5}\right)$ of the output neurons for the five samples $\left(S_{1}, \cdots, S_{5}\right)$ and each column represents the response of all output neurons for a given sample. The numbers in brackets on the left 
represent the associated class for that neuron and those at the bottom represent the actual class label for that sample. It can be observed from the figure that after training, the network has one neuron, from class one and two neurons each from classes two and three. The predicted class for a given sample is determined based on the class association of the output neuron that fires first. Using this mechanism for class determination, it can be observed that the predicted class labels for all samples are correctly estimated except the fifth sample $\left(S_{5}\right)$. Further, for the second sample $\left(S_{2}\right)$, the third neuron fires close to the target firing time. On the other hand, the class label for the sample three is correctly predicted but the associated neuron fires much later than the target firing time. Next, the performance of the SRESN classifier for the Iris flower classification problem is

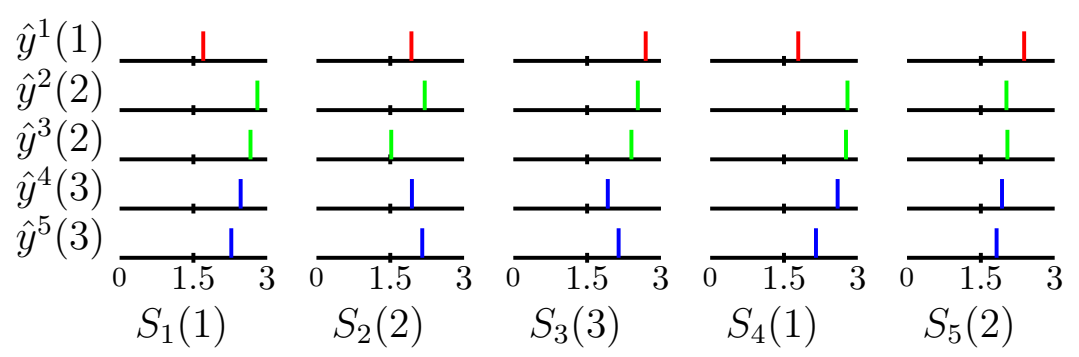

Figure 3.13: Firing time of neurons for five random testing samples from Iris flower classification dataset

Table 3.1: Performance comparison of SRESN with existing spiking classifiers - Fisher Iris problem

\begin{tabular}{|c|c|c|c|c|}
\hline Algorithm & Architecture & $\begin{array}{c}\text { \# Network } \\
\text { Parameters }\end{array}$ & $\begin{array}{c}\text { Testing } \\
\text { Accuracy (\%) }\end{array}$ & $\begin{array}{c}\# \\
\text { Epochs }\end{array}$ \\
\hline SRESN & $\mathbf{2 0 - 5}$ & $\mathbf{1 0 0}$ & $\mathbf{9 7 . 0 3 ( 0 . 7 6 )}$ & $\mathbf{2 9 7}$ \\
SpikeProp & $49-10-3$ & 24000 & $96.1(0.1)$ & 1000 \\
MuSpiNN & $17-8-1(3)^{1}$ & 576 & 95.87 & 192 \\
SWAT & $16-208-3$ & 624 & $95.3(3.6)$ & 500 \\
Multi-spike & $21-8-1$ & 880 & 94.72 & 241 \\
(GMES) & & & & \\
Multi-spike & $21-8-1$ & 880 & 93.98 & 234 \\
(arbitrary spike) & & & & \\
eSNN & $120-84$ & 10080 & 95 & 1 \\
\hline
\end{tabular}

${ }^{1}$ MuSpiNN converts the three class problem into three binary problems. Hence, three networks are used, one for each problem. The number of network parameters for a single neural network have been provided. 
compared with the that of other algorithms.

Table 3.1 shows the results of performance evaluation of the different learning algorithms for the Iris flower classification problem. The table displays details regarding the network architecture, number of network parameters, overall testing accuracy and the number of epochs required for each algorithm. The difference in the number of input neurons is because the different algorithms use different number of receptive fields to encode the input features. SpikeProp and MuSpiNN employ 12 and 4 receptive fields per feature, respectively with a single bias neuron. Both SRESN and Multi-spike learning employ 5 receptive field neurons per feature but Multi-spike learning uses an extra bias neuron. SWAT and eSNN employ 4 and 30 receptive fields per feature, respectively. The average execution time per epoch of the SRESN classifier for the first 100 epochs was 0.25 seconds. It could be observed from the table that the SRESN classifier achieves an improvement of 1 - 3\% over other algorithms. Further, the SRESN classifier achieves this improvement using significantly lesser network parameters than the other algorithms. This is due to the fact that the SRESN classifier is evaluated using population coding scheme without any delays, resulting in fewer network parameters.

\subsubsection{Wisconsin Breast Cancer Problem}

In this problem, the goal of the learning algorithm is to classify each sample as a case of malign or benign cancer. The data set has a total of 686 samples (16 samples with

Table 3.2: Performance comparison of the SRESN classifier with existing spiking classifiers - Wisconsin Breast Cancer problem.

\begin{tabular}{|c|c|c|c|c|}
\hline Algorithm & Architecture & $\begin{array}{c}\text { \# Network } \\
\text { Parameters }\end{array}$ & $\begin{array}{c}\text { Testing } \\
\text { Accuracy (\%) }\end{array}$ & $\begin{array}{c}\# \\
\text { Epochs }\end{array}$ \\
\hline SpikeProp & $64-15-3$ & 30720 & $97.0(0.6)$ & 1500 \\
SWAT & $9-117-2$ & 234 & $96.7(2.3)$ & 500 \\
SRESN & $\mathbf{4 5 - 2}$ & $\mathbf{9 0}$ & $\mathbf{9 6 . 4 ( 0 . 4 8 )}$ & $\mathbf{2 8 5}$ \\
Multi-spike & $46-8-1$ & 1880 & 95.32 & 209 \\
(GMES) & & & & \\
MuSpiNN & $46-8-1$ & 1880 & 94.66 & 166 \\
eSNN & $135-280$ & 37800 & 98.7 & 1 \\
\hline
\end{tabular}


missing values are excluded), and each sample consists of 9 features. Similar to the Iris flower classification problem, ten samples from each class are used for training and the rest are used for testing. The values for the parameters $T_{C C}$ and $T_{M C}$ are set to $2.7 \mathrm{~ms}$ and $2.4 \mathrm{~ms}$ respectively.

Table 3.2 displays the results of performance evaluation of the different algorithms along with network architecture, the number of network parameters and the number of epochs for each algorithm. In this case, SpikeProp employs 8 receptive fields per feature with a single bias neuron. SRESN, MuSpiNN and Multi-spike learning employ 5 receptive field neurons per feature but MuSpiNN and Multi-spike learning also uses an extra bias neuron. SWAT uses a single neuron per feature whereas eSNN employs 15 receptive fields per feature, respectively. The average execution time per epoch of the SRESN classifier for the first 100 epochs was 1.4 seconds. It can be observed from the table that the results of the SRESN classifier are similar to the performance evaluation results of SpikeProp, SWAT and Multi-Spike (GMES). But, the SRESN classifier requires much lesser network parameters in comparison to the these algorithms. With regards to eSNN, the performance of eSNN is $2 \%$ better than that of the SRESN classifier. But, eSNN requires a large number of network parameters to achieve this improvement in performance.

\subsection{Discussion on the Limitations of the SRESN clas- sifier}

The self-regulated approach to learning helps the SRESN classifier in closely approximating the relationship between the input samples and the corresponding class labels. But, the SRESN classifier has certain limitations which are discussed below:

- Single spike encoding: An important attribute of the human brain is that information can be encoded using multiple spikes with different spike times. This allows faster and accurate transmission of information. However, the SRESN classifier is limited to handling data that is encoded using single spikes only.

The SRESN classifier employs rank order learning for initializing the weights of 


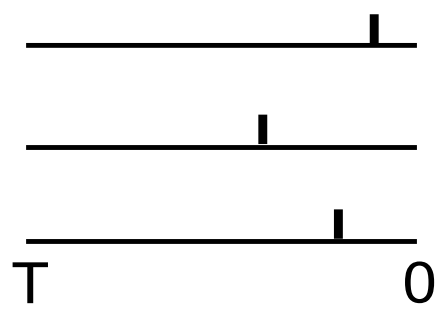

(a)

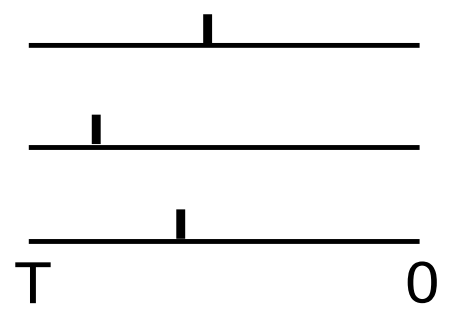

(b)

Figure 3.14: Two spike patterns with different spike times for which rank order learning would estimate equal weights

newly added neurons. Rank order learning is based on the assumption that the most important information about a stimulus is contained in the first spikes generated by the neurons. It does not consider the subsequent spikes for estimating the network parameters. As a consequence, the SRESN classifier can not utilize information present in multiple spikes.

- The precise time of the spikes are ignored: Rank order learning initializes the weights of the newly added neurons using the order of spikes. It does not consider the precise time of spikes for initializing the weights of a given neuron. This renders rank order learning an unsuitable technique for weight initialization when two different spike patterns follow the same order but differ considerably in terms of spike times.

Figure 3.14 shows example of two spike patterns with considerably different spike times for which rank order learning will calculate equivalent weights. In this case, rank order learning may not be able to accurately capture the information present in the input spike pattern.

\subsection{Summary}

In this chapter, a Self-Regulating Evolving Spiking Neural (SRESN) classifier was presented. The SRESN classifier starts with no output neurons and evolves the network architecture during the training process. It regulates its learning strategies for each sample depending on the information present in that sample with respect to the knowledge stored in the network. It can choose to add a neuron or update the network parameters 
or skip learning a sample. It uses rank order learning to initialize the weights of newly added neurons to the network. The rank order learning takes into account the global information in the network, but it limits the application of the SRESN classifier to problems where each presynaptic neuron generates a single spike in the simulation interval.

In the next chapter, a two stage margin maximization spiking neural network is presented that utilizes the locally available information to update the synaptic weights. 
Chapter 3. SRESN ClASSIFIER 


\section{Chapter 4}

\section{A Two Stage Margin Maximization Spiking Neural Network}

In the previous chapter, a Self-Regulating Evolving Spiking Neural (SRESN) classifier was developed that employed heuristic techniques to evolve the network architecture and update the synaptic weights using improved rank order learning. The SRESN classifier employs rank order learning for initializing and updating the synaptic weights in the network. Rank order learning is based on the order of first spikes generated by the presynaptic neurons. It ignores the precise time of the spikes. Further, it can only be used when the input features are represented using single spikes in the simulation interval. To overcome these issues, a Two stage Margin Maximization Spiking Neural Network (TMM-SNN) is developed in this chapter.

TMM-SNN employs a three layered spiking neural network architecture. It uses a two stage learning algorithm to update the synaptic weights in the network. In the first stage (structure learning stage), the learning algorithm evolves the hidden layer and estimates the synaptic weights for the hidden layer neurons. The weights for the newly added neurons are initialized using a new Activation Based Coding (ABC) scheme that is developed in this thesis. The ABC scheme uses locally available information present in multiple spikes generated by the presynaptic neurons to initialize the synaptic weights of a newly added neuron such that it fires at a precise time. The weights for the hidden neurons are updated using a margin maximization strategy that maximizes the separation between the spike times of interclass neurons. At the end of the structure 
learning stage, the weights for the hidden layer neurons are fixed. Based on the structure determined in the first stage, the synaptic weights for the connections between the hidden and output layer neurons are randomly initialized. In the second stage (output weights learning stage), the learning algorithm estimates the synaptic weights for the output layer neurons using a margin maximization strategy.

To analyze the working of the TMM-SNN and the effect of its algorithm parameters, a performance study is conducted using the Ionosphere problem from the UCI machine learning repository. Based on this study, certain guidelines are also discussed for initializing the algorithm parameters appropriately. The performance of the learning algorithm has been compared with existing batch learning algorithms for spiking neural networks, viz. SpikeProp [17], synaptic weight association training [10] and the SRESN classifier for ten benchmark data sets from the UCI machine learning repository. The results of the performance evaluation have been statistically compared using the one-way ANOVA test [22] followed by a pairwise comparison using the Fisher's least significant difference method [23]. The results of performance evaluation clearly indicate that the TMMSNN can achieve better generalization performance in comparison to existing learning algorithms for spiking neural networks using a compact network architecture in a 95\% confidence interval.

\subsection{Two Stage Margin Maximizing Spiking Neural Network for Classification Problems}

In this section, the architecture and the evolving learning algorithm for the Two stage Margin Maximizing Spiking Neural Network (TMM-SNN) for classification problems is presented. TMM-SNN is a three layered neural network consisting of spiking neurons. The learning algorithm for TMM-SNN employs a two stage approach to learning. In the first stage (referred to as the structure learning stage), the learning algorithm evolves and updates the weights of the hidden layer neurons. In the second stage (referred to as the output weights learning stage), the learning algorithm updates the weights for the output layer neurons. Both stages of the learning algorithm continue for multiple epochs until convergence is achieved. 
Without loss of generality, in the following description it is assumed that the network has grown to $K$ neurons in the hidden layer after training for $(n-1)$ spike patterns.

\subsubsection{Architecure of TMM-SNN}

Figure 4.1 shows the architecture of a three layered TMM-SNN. The training spike patterns $\left\{\left(\mathbf{x}_{1}, c_{1}\right), \cdots,\left(\mathbf{x}_{n}, c_{n}\right), \cdots,\left(\mathbf{x}_{N}, c_{N}\right)\right\}$ are presented to the network, one by one, at intervals of time $T$, where $T$ is termed as the simulation interval. The sample $\mathbf{x}_{n}=\left[x_{n}^{1}, \cdots, x_{n}^{i}, \cdots, x_{n}^{m}\right]$ is an $m$-dimensional spike pattern and $c_{n} \in\{1, \cdots, C\}$ is its class label. Here, $C$ is the total number of classes. Thus, the network has $m$ input neurons and $C$ output neurons. The input feature, $x_{n}^{i}=\left\{t_{i}^{(1)}, \cdots, t_{i}^{(g)}, \cdots, t_{i}^{\left(G_{i}\right)}\right\}$, is a spike train with $G_{i}$ spikes, where $t_{i}^{(g)}$ represents the $g^{\text {th }}$ spike generated by the $i^{\text {th }}$ input neuron.

For a given input spike pattern, the response of the $k^{\text {th }}$ hidden neuron is a function of the Postsynaptic Potential (PSP) induced by the input neurons. The postsynaptic potential $\left(v_{1}^{i}\right)$ induced by the $i^{\text {th }}$ input neuron at time $t$ is given by

$$
v_{1}^{i}(t)=\sum_{g} \epsilon\left(t-t_{i}^{(g)}\right)
$$

where $\epsilon\left(t-t_{i}^{(g)}\right)$ is the postsynaptic potential at time $t$ induced by a presynaptic spike at $t_{i}^{(g)}$ on a synapse with weight one. In this work, $\epsilon($.$) has been modeled using the spike$

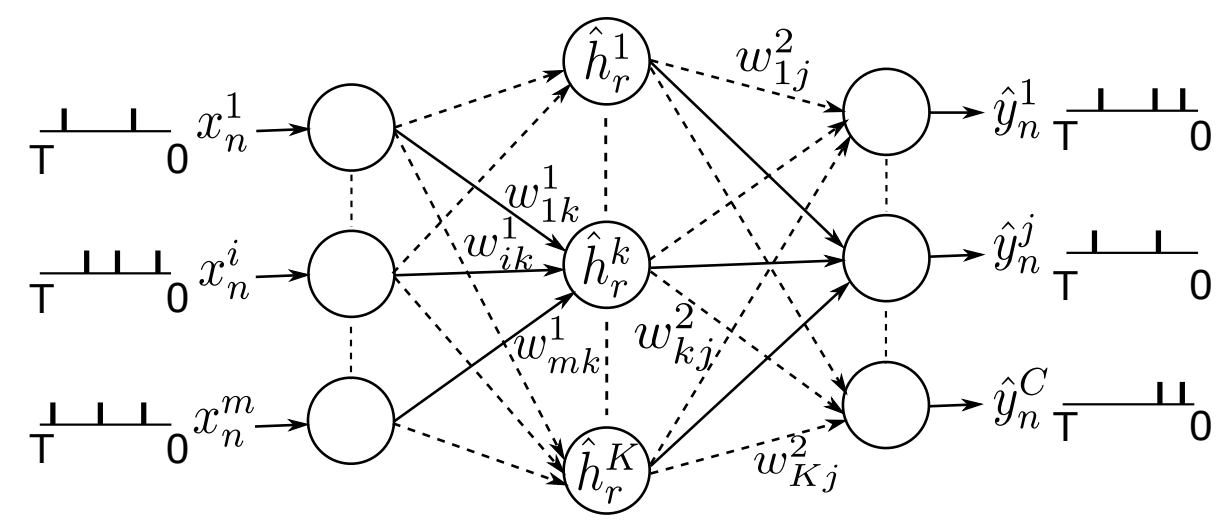

Figure 4.1: Architecture of TMM-SNN 
response function [17], given by

$$
\epsilon(s)=\frac{s}{\tau} e^{\left(1-\frac{s}{\tau}\right)}
$$

where $\tau$ is the time constant for the neuron.

From Equation (4.1), the postsynaptic potential $\left(v_{k}^{1}\right)$ of the $k^{t h}$ hidden neuron at time $(t)$ is given as

$$
v_{k}^{1}(t)=\sum_{i} w_{i k}^{1} v_{1}^{i}(t)
$$

where $w_{i k}^{1}$ is the weight of the synapse between the $i^{\text {th }}$ input neuron and the $k^{\text {th }}$ hidden neuron. If $\theta_{k}^{1}$ represents the threshold of the $k^{\text {th }}$ hidden neuron, then the $k^{\text {th }}$ hidden neuron generates a spike whenever its PSP crosses this threshold. After generating a spike, the PSP of the $k^{\text {th }}$ hidden neuron is reset to zero. The response of the $k^{\text {th }}$ hidden neuron for $\mathbf{x}_{n}$ is also a spike train, denoted by $h_{n}^{k}=\left[\bar{t}_{k}^{(1)}, \cdots, \bar{t}_{k}^{(g)}, \cdots, \bar{t}_{k}^{\left(G_{k}\right)}\right]$. Based on the response of the hidden layer neurons, the postsynaptic potential $\left(v_{2}^{k}\right)$ induced by the $k^{\text {th }}$ hidden neuron at time $t$ is given by

$$
v_{2}^{k}(t)=\sum_{g} \epsilon\left(t-\bar{t}_{k}^{(g)}\right)
$$

The PSP $\left(v_{j}^{2}\right)$ of the $j^{\text {th }}$ output layer neuron depends on the PSP induced by the hidden layer neurons and is given by

$$
v_{j}^{2}(t)=\sum_{k} w_{k j}^{2} v_{2}^{k}(t)
$$

where $w_{k j}^{2}$ is the weight of the synapse between the $k^{\text {th }}$ hidden neuron and the $j^{\text {th }}$ output neuron. The threshold for all the output neurons is set to one. Thus, the $j^{\text {th }}$ output neuron will generate a spike whenever its PSP $\left(v_{j}^{2}\right)$ is higher than one, and its PSP is reset to zero immediately after generating a spike. The response of the $j^{\text {th }}$ output neuron is a spike pattern $\hat{y}_{n}^{j}=\left[\hat{t}_{j}^{(1)}, \cdots, \hat{t}_{j}^{(g)}, \cdots, \hat{t}_{j}^{\left(G_{j}\right)}\right]$.

The predicted class for a given input spike pattern is determined (encoded) according to the output neuron that fires first. Since a correct prediction depends only on the time of the first spike generated by the output layer neurons, the learning algorithm utilizes only the time of the first spike generated by the hidden and output layer neurons in the learning process. For convenience, the time of the first spike generated by the $k^{\text {th }}$ 


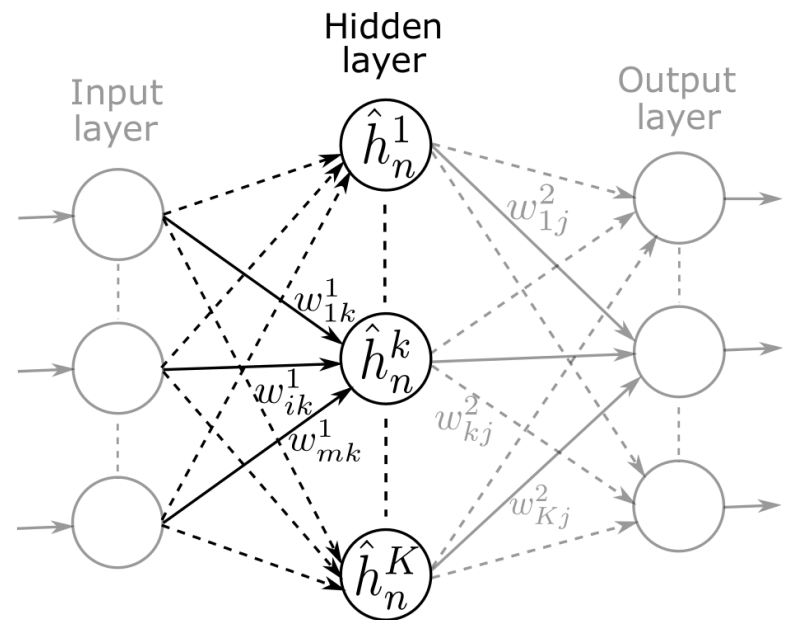

(a) Structure learning stage

Hidden

layer

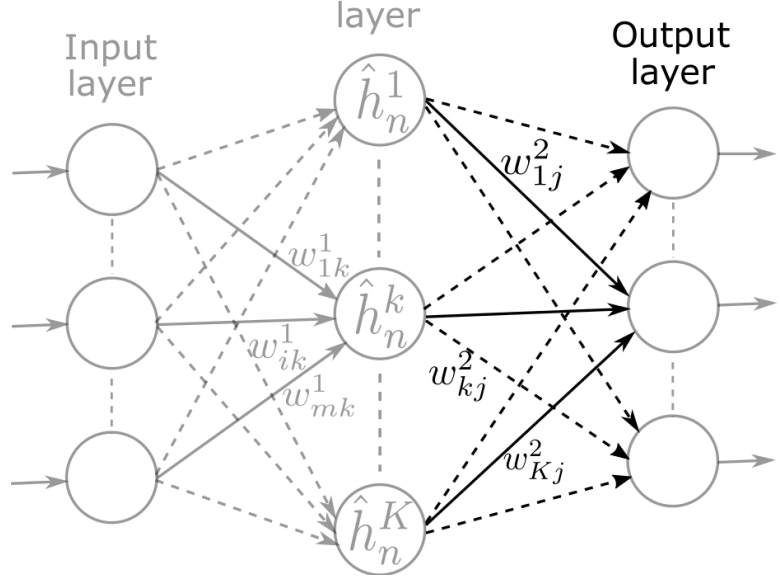

(b) Output weights learning stage

Figure 4.2: The two stages of the learning algorithm for TMM-SNN. In the structure learning stage, the learning algorithm evolves the hidden layer neurons and updates the weights for synaptic connections between input and hidden layer neurons. In the output weights learning stage, the learning algorithm updates the weights for output layer neurons.

hidden neuron and the $j^{\text {th }}$ output neuron are represented as $\bar{t}_{k}$ and $\hat{t}_{j}$, respectively. The objective of the learning algorithm for TMM-SNN is to closely approximate the functional relationship between the input spike patterns and the corresponding class labels so that the correct class output neuron fires first for a given spike pattern. 


\subsubsection{Learning Algorithm for TMM-SNN}

The learning algorithm for TMM-SNN employs a two stage approach to learning. Figure 4.2 diagrammatically shows the parameters adjusted in the two stages of the learning algorithm for TMM-SNN. In the first stage, termed as the structure learning stage, the learning algorithm determines the appropriate network architecture for a given problem by evolving the hidden layer neurons. Also, in this stage, the learning algorithm updates the weights of the synaptic connections between the input and the hidden layer neurons. The highlighted portion of the Figure 4.2a depicts the parameters adjusted in the structure learning stage. In the second stage, termed as the output weights learning stage, the learning algorithm projects the response of hidden neurons back to the $C$-dimensional output space. For this purpose, the learning algorithm updates the weights for the output layer neurons and the weights for the hidden layer neurons are not adjusted in this stage. The highlighted parts of the Figure $4.2 \mathrm{~b}$ reflect the parameters adjusted in the output weights learning stage. A brief overview of each stage of the training process is provided below followed by a detailed description of the different learning strategies used in the two stages.

1) Structure Learning stage: In this stage, the learning algorithm evolves and updates the weights of the hidden layer neurons. It employs the 'neuron addition strategy' to determine the appropriate number of hidden neurons and their class associations for a given problem in the first epoch. The weights of the newly added neurons are initialized using the activation based coding scheme. Further, the learning algorithm updates the weights of the hidden layer neurons so that the hidden neuron from the same class as the current input spike pattern fires first with a high interclass margin. For this purpose, a target firing time is defined for each of the hidden layer neurons. Suppose, $c_{k}$ represents the class association of the $k^{t h}$ hidden neuron, then its target firing time $\left(t_{k}\right)$ for a given spike pattern is defined as

$$
t_{k}= \begin{cases}T_{h} & c_{k}=c_{r} \\ T+\delta & c_{k} \neq c_{r}\end{cases}
$$

where $T_{h} \in[0, T]$ is a fixed time instant in the simulation interval. $\delta$ is small positive number and a target firing time of $(T+\delta)$ implies that the particular hidden neuron 
should not generate a spike in the simulation interval for the current input spike pattern. The weights of the hidden layer neurons are updated using the 'margin maximization strategy in the structure learning stage' for multiple epochs. In each epoch, the learning algorithm updates the synaptic weights for the connection between input neurons and the first firing hidden layer neurons from the same class as the current input spike pattern and a different class. The weights for the neurons are updated such that the temporal difference (interclass margin) between their spike times is maximized for each training spike pattern. This strategy for margin maximization is used for multiple epochs and the structure learning stage ends when the mean interclass margin based on the output of the hidden neurons for all training spike patterns converges.

2) Output Weights Learning Stage: In this stage, the learning algorithm uses the "margin maximization strategy in the output weights learning stage' to update the weights of the output layer neurons. For a given spike pattern, the weights of the output layer neurons are updated such that the correct class output neuron fires first with high interclass margin. In this stage, the learning algorithm updates the synaptic weights for the connection between output neurons and the first firing hidden neurons from the same class as the current input spike pattern. The weights of the neurons are updated such that the interclass margin based on the spike times of output neurons for each input spike pattern is maximized. This stage also continues for multiple epochs until the mean interclass margin for all the training spike patterns converges.

Next, a detailed description of the learning strategies used in the two stages is presented.

\subsubsection{Structure Learning Stage}

In this section, the 'neuron addition strategy' and the 'margin maximization strategy for the structure learning stage' are described. In the following description, $C C$ refers to the hidden neuron that fires first and is associated with the same class as the current input spike pattern. Based on this definition, $C C$ is given by

$$
C C=\underset{k, c_{k}=c_{r}}{\operatorname{argmin}} \bar{t}_{k}
$$


Similarly, $M C$ refers to the hidden neuron associated with any other class that first first and is given by

$$
M C=\underset{k, c_{k} \neq c_{r}}{\operatorname{argmin}} \bar{t}_{k}
$$

The time of first spike generated by the hidden neurons $C C$ and $M C$ are represented as $\bar{t}_{C C}$ and $\bar{t}_{M C}$, respectively.

Neuron addition strategy: A new hidden neuron is added to the network when none of the existing neurons, associated with the class $c_{n}$, generate a spike closer to the target firing time $\left(T_{h}\right)$. A late spike by all the existing hidden neurons implies that the knowledge stored in the network is not enough to approximate the information present in the current input spike pattern. To add a neuron to the network for a given spike pattern, a fixed time instant $T_{a} \in\left[T_{h}, T\right]$ is used as a threshold for $\bar{t}_{C C}$, such that if $\bar{t}_{C C}$ is greater than $T_{a}$, then a neuron is added to the hidden layer in the network. Thus, the criterion for adding a neuron is given as

$$
\text { If } \bar{t}_{C C}>T_{a}
$$

Then add a neuron to the network

where $T_{a}$ is given as

$$
T_{a}=\alpha_{a} T+\left(1-\alpha_{a}\right) T_{h}
$$

Here, $\alpha_{a}$ is termed as the addition threshold and is always set in the interval $[0,1]$. When $\alpha_{a}$ is set to one, $T_{a}$ is equal to $\mathrm{T}$; as a result, fewer neurons are added to the network. This leads to thedevelopment of an inaccurate model of the data. When $\alpha_{a}$ is set to zero, $T_{a}$ is equal to $T_{h}$ as a result a neuron is added to the network for each input spike pattern. This may lead to overfitting, resulting in poor generalization on unseen spike patterns. Therefore, the value for $\alpha_{a}$ needs to be chosen carefully and a suitable range for setting $\alpha_{a}$ is the interval $[0.15,0.35]$. The suitable range for $\alpha_{a}$ is chosen by conducting studies that illustrate the effect of $\alpha_{a}$ on the network structure and the training/testing accuracies of TMM-SNN. The results of these studies have been provided in Section 4.2 in the thesis.

The weights for the newly added neuron are initialized using a new encoding scheme, 


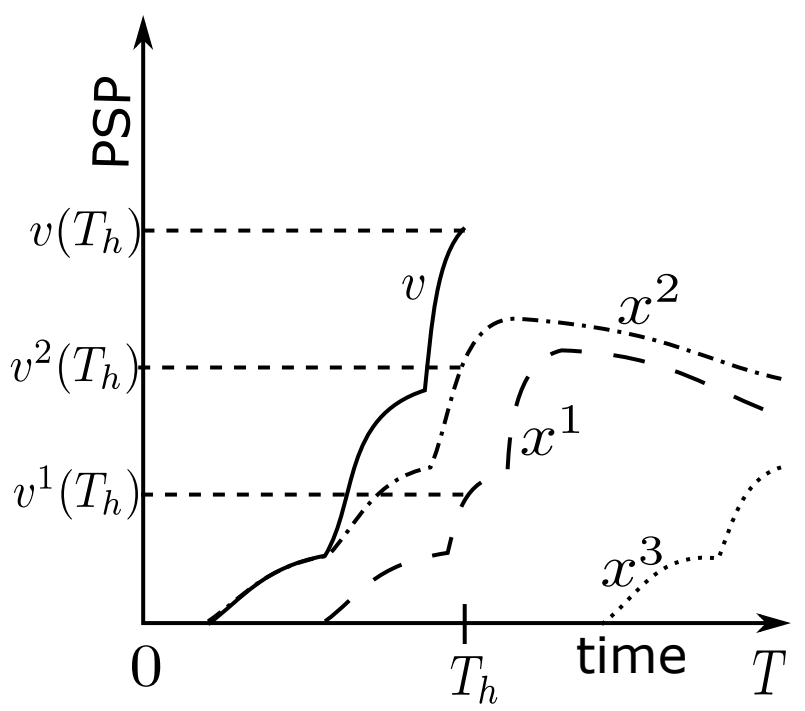

Figure 4.3: An illustrative example of the activation based coding with three input neurons

termed as the Activation Based Coding (ABC). For a given input spike pattern, the ABC technique initializes the weights of a newly added neuron using the normalized PSP of the input neurons at the target firing time $\left(T_{h}\right)$. The normalized PSP $\left(u_{i}^{1}\right)$ for the $i^{t h}$ input neuron at time $t$ is equal to the fraction of total PSP, that is induced by the $i^{\text {th }}$ input neuron, given as

$$
u_{i}^{1}(t)=\frac{v_{1}^{i}(t)}{\sum_{i} v_{1}^{i}(t)}
$$

It represents the information contained in the spike train generated by $i^{\text {th }}$ input neuron with respect to the total information present in the current input spike pattern. From Equation (4.11), the weights for the newly added neuron are initialized using the normalized PSP of the corresponding input neuron at the target firing time $\left(T_{h}\right)$ as:

$$
w_{i(K+1)}^{1}=u_{i}^{1}\left(T_{h}\right), \quad i \in\{1, \cdots, m\}
$$

Figure 4.3 show an illustrative example with three input features $\left(\mathbf{x}=\left[x^{1}, x^{2}, x^{3}\right]\right)$ depicting the use of the $\mathrm{ABC}$ technique to initialize the weights of a postsynaptic neuron. In the figure, the PSP induced by the three input neurons in the interval $[0, T]$ is represented by $v^{1}, v^{2}$ and $v^{3}$, respectively. The total PSP of the postsynaptic neuron during the interval $[0, T]$ is denoted by $v$. It may be observed from the figure that the inputs 
$x^{1}$ and $x^{2}$ have a non-zero contribution to PSP at $T_{h}$ whereas $x^{3}$ has zero contribution to PSP at $T_{h} \cdot v^{1}\left(T_{h}\right)$ and $v^{2}\left(T_{h}\right)$ represent the PSP due to the input features $x^{1}$ and $x^{2}$ at $T_{h}$, respectively and $v\left(T_{h}\right)$ is the total PSP of the newly added neuron at $T_{h}$. Thus, the weights for the three input neurons using the $\mathrm{ABC}$ technique will be initialized to $\left[v^{1}\left(T_{h}\right) / v\left(T_{h}\right), v^{2}\left(T_{h}\right) / v\left(T_{h}\right), 0\right]$.

The threshold for the newly added neuron is initialized as

$$
\theta_{(K+1)}^{1}=\sum_{i} w_{i(K+1)}^{1} v_{1}^{i}\left(T_{h}\right)
$$

The initialization of the weights and threshold for the newly added neuron using Equations (4.12) and (4.13) ensures that it fires precisely at the target firing time $\left(T_{h}\right)$ for the current input spike pattern.

Margin maximization strategy for structure learning stage: The learning algorithm for TMM-SNN uses this strategy to improve the generalization performance of the network by maximizing the interclass margin. The concept of margin maximization is inspired by the concept of support vector machines as maximum margin classifiers [84]. In case of TMM-SNN, the interclass margin $\left(\Upsilon_{[n]}^{1}\right)$ for the current sample $\left(\mathbf{x}_{n}\right)$ is defined as the temporal distance between the spike times of the neurons $C C$ and $M C$, given as

$$
\Upsilon_{[n]}^{1}=\bar{t}_{M C}-\bar{t}_{C C}
$$

A smaller interclass margin, for a given spike pattern, indicates that the particular spike pattern is close to the decision boundary. This may lead to poor generalization performance on unseen spike patterns. To identify the spike patterns with a smaller margin, the learning algorithm uses a time duration, $T_{m} \in\left[T_{h}, T\right]$ as a threshold for $\Upsilon_{[n]}^{1}$. Thus, the criterion for this strategy is given as

$$
\text { If } \Upsilon_{[n]}^{1}<T_{m}
$$

Then update the weights for existing neurons

where $T_{m}$ is given as

$$
T_{m}=\alpha_{m}\left(T-T_{h}\right)
$$


Here $\alpha_{m}$ is termed as the margin threshold and it is always set in the interval $[0,1]$. A higher value of margin threshold will result in the loss of information gained from the past spike patterns. On the other hand, a lower value of margin threshold may result in poor generalization on the testing spike patterns. It was observed that the performance of the learning algorithm is satisfactory when $\alpha_{m}$ is set in the interval $[0,0.1]$. For the simulation results reported in this work, $\alpha_{m}$ is fixed at 0.07 .

To update the margin for a given spike pattern, the learning algorithm may choose to update the weights of the neuron $M C$ alone or both $C C$ and $M C$. The weights of the neuron $M C$ alone are updated when $\bar{t}_{C C}$ is closer to $T_{h}$, otherwise the learning algorithm updates the weights for both the neurons. When $C C$ fires closer to $T_{h}$, it indicates that the current input spike pattern is similar to the previously learned spike patterns. This implies that the network can approximate the information present in the current input spike pattern, but the spike pattern is closer to the decision boundary. In such a scenario, the learning algorithm updates the neuron $M C$ without updating the neuron $C C$. Similarly, when $C C$ fires much later than $T_{h}$, the learning algorithm updates the weights for both the neurons $C C$ and $M C$. To determine, if $C C$ fired closer to $T_{h}$ for a given spike pattern, the learning algorithm uses a time instant $T_{u} \in\left[T_{h}, T\right]$ as a threshold for $\bar{t}_{C C}$. Thus, the learning algorithm updates the weights for the neuron $M C$ when $\bar{t}_{C C}$ is lower than $T_{u}$, otherwise the weights for both the neurons are updated. Here, $T_{u}$ is given as

$$
T_{u}=\alpha_{u} T+\left(1-\alpha_{u}\right) T_{h}
$$

where $\alpha_{u}$ is termed as the similarity threshold and is always set in the interval $[0,1]$. If $\alpha_{u}$ is set to 1 , then $T_{u}$ will be equal to $\mathrm{T}$. In this case, the weights of the neurons $C C$ are never updated which may result in the generation of an inaccurate model of data. When $\alpha_{u}$ is set to $0, T_{u}$ will be equal to $T_{h}$ as a result, the learning algorithm always updates both the neuron $C C$ and $M C$. This may cause over-fitting as the learning algorithm learns similar samples repeatedly resulting in lower generalization performance. Hence, $T_{u}$ should be set closer to $T_{h}$ to generate an accurate model of data that generalizes properly on unseen data. For this purpose, the simulations presented in this chapter use a value of 0.07 for $\alpha_{u}$.

The PSP induced by the input neurons provide an estimate of the information present 
in the current input spike pattern. Further, the information from the past spike patterns is stored in the synaptic weights of the hidden layer neurons. To update the weights of the $k^{\text {th }}$ hidden neuron, the learning algorithm considers both the past knowledge stored in the network as well as the information present in the current input spike pattern. The weights of a given synapse are updated in proportion to the difference between its existing weights and the normalized PSP of the corresponding input neuron at $\bar{t}_{k}$. Further, the learning algorithm updates the weights of only those synapses, whose existing weights are lower that the normalized PSP of the corresponding input neuron at $\bar{t}_{k}$. This implies that the weights of the synapses with higher contribution to PSP for past spike patterns are not updated, thereby preserving the existing knowledge stored in the network. Based on this, the change in weights $\left(\Delta w_{i(C C)}^{1}\right)$ of the neuron $C C$ is given as

$$
\Delta w_{i(C C)}^{1}= \begin{cases}0 & w_{i(C C)}^{1} \geq u_{i}^{1}\left(\bar{t}_{C C}\right) \\ \eta\left(u_{i}^{1}\left(\bar{t}_{C C}\right)-w_{i(C C)}^{1}\right) & w_{i(C C)}^{1}<u_{i}^{1}\left(\bar{t}_{C C}\right)\end{cases}
$$

where $\eta$ is the learning rate and is set to 0.01 in this chapter to avoid oscillations in the learning process. It may be observed from the above equation that, the weight update can be executed locally as it relies only on the normalized PSP induced by the input neurons and the existing weights of the corresponding synapses. Similarly, the change in weights $\left(\Delta w_{i(M C)}^{1}\right)$ of the neuron $M C$ is given as

$$
\Delta w_{i(M C)}^{1}= \begin{cases}0 & w_{i(M C)}^{1} \geq u_{i}^{1}\left(\bar{t}_{M C}\right) \\ \eta\left(w_{i(M C)}^{1}-u_{i}^{1}\left(\bar{t}_{M C}\right)\right) & w_{i(M C)}^{1}<u_{i}^{1}\left(\bar{t}_{M C}\right)\end{cases}
$$

Updating the weights of the neuron $C C$ and $M C$ using Equations (4.18) and (4.19) helps in maximizing the interclass margin, thereby improving the generalization performance of the learning algorithm. Next, the learning strategy used in output weights learning stage will be described. 


\subsubsection{Output weights learning stage}

In this section, the 'margin maximization strategy in the output weights learning stage' is described. For this stage, $C C$ refers to the output neuron associated with the same class as the current input spike pattern and the time of the first spike generated by $C C$ is represented by $\hat{t}_{C C}$. $M C$ refers to the neuron that fires first and is associated with any other class, given as

$$
M C=\underset{j, j \neq c_{r}}{\operatorname{argmin}} \hat{t}_{j}
$$

The time of first spike generated by the neuron $M C$ is represented as $\hat{t}_{M C}$.

Margin maximization strategy in output weights learning stage: The purpose of the margin maximization strategy in the output weights learning stage is same as its role in the structure learning stage. It tries to maximize the interclass margin for the neurons $C C$ and $M C$. In this stage, the interclass margin $\left(\Upsilon_{[n]}^{2}\right)$ for the current input spike pattern $\left(\mathbf{x}_{n}\right)$ is defined as

$$
\Upsilon_{[n]}^{2}=\hat{t}_{M C}-\hat{t}_{C C}
$$

The learning algorithm updates the weights for the neurons $C C$ and $M C$ when $\Upsilon_{[n]}^{2}$ is lower than the threshold $T_{m}$. Thus, the criterion for updating the weights of the neurons $C C$ and $M C$ is given by

$$
\text { If } \Upsilon_{[n]}^{2}<T_{m}
$$

Then update the weights for the neurons CC and MC

After the structure learning stage, the correct class hidden layer neuron fires first for most of the training spike patterns. Essentially, the output weights learning stage is used by the learning algorithm to map the output of hidden neurons back to the $C$ dimensional output space. For this purpose, the weights for the connections between the hidden neurons from the same class as the current input spike pattern and the output neuron are alone updated. The weights for the output layer neurons are updated using a learning rule similar to the learning rule described in Equations (4.18) and (4.19). It uses 
the normalized PSP $\left(u_{k}^{2}\right)$ induced by the $k^{\text {th }}$ hidden layer neuron at $\hat{t}_{j}$ which is given by

$$
u_{k}^{2}\left(\hat{t}_{j}\right)=\frac{v_{2}^{k}\left(\hat{t}_{j}\right)}{\sum_{k} v_{2}^{k}\left(\hat{t}_{j}\right)}
$$

Based on the normalized PSP, the change in weights $\left(\Delta w_{k(C C)}^{2}\right)$ of the neuron $C C$ in the output layer is given as

$$
\Delta w_{k(C C)}^{2}=\left\{\begin{array}{ll}
\eta\left(u_{k}^{2}\left(\hat{t}_{C C}\right)-w_{k(C C)}^{2}\right) & \left(w_{k(C C)}^{2}<u_{k}^{2}\left(\hat{t}_{C C}\right)\right) \&\left(c_{k}=c_{r}\right) \\
0 & \text { otherwise }
\end{array}, \quad k \in\{1, \cdots, K\}\right.
$$

It may be observed from the above equation that the weights of the output layer neurons are also updated using only the locally available information. Similarly, the change in weights $\left(\Delta w_{k(C C)}^{2}\right)$ of the neuron $M C$ is given as

$\Delta w_{k(M C)}^{2}=\left\{\begin{array}{ll}\eta\left(w_{k(M C)}^{2}-u_{k}^{2}\left(\hat{t}_{M C}\right)\right) & \left(w_{k(M C)}^{2}<u_{k}^{2}\left(\hat{t}_{M C}\right)\right) \&\left(c_{k}=c_{r}\right) \\ 0 & \text { otherwise }\end{array}, \quad k \in\{1, \cdots, K\}\right.$

To summarize, the learning algorithm employs the strategies of neuron addition, margin maximization for structure learning and output weights learning to closely approximate the relationship between the input spike patterns and the corresponding class labels using a compact network architecture. The learning algorithm for TMM-SNN has been summarized in a pseudocode format in Algorithm 1.

\subsection{Working of TMM-SNN Using the Ionosphere Prob- lem}

In this section, the Ionosphere problem from the UCI machine learning repository is used to illustrate the working of the margin maximization strategy and study the effect of the addition threshold $\left(\alpha_{a}\right)$ on the performance of TMM-SNN. Based on this study, suitable guidelines are provided for selecting $\alpha_{a}$. The performance of the TMM-SNN is computed 


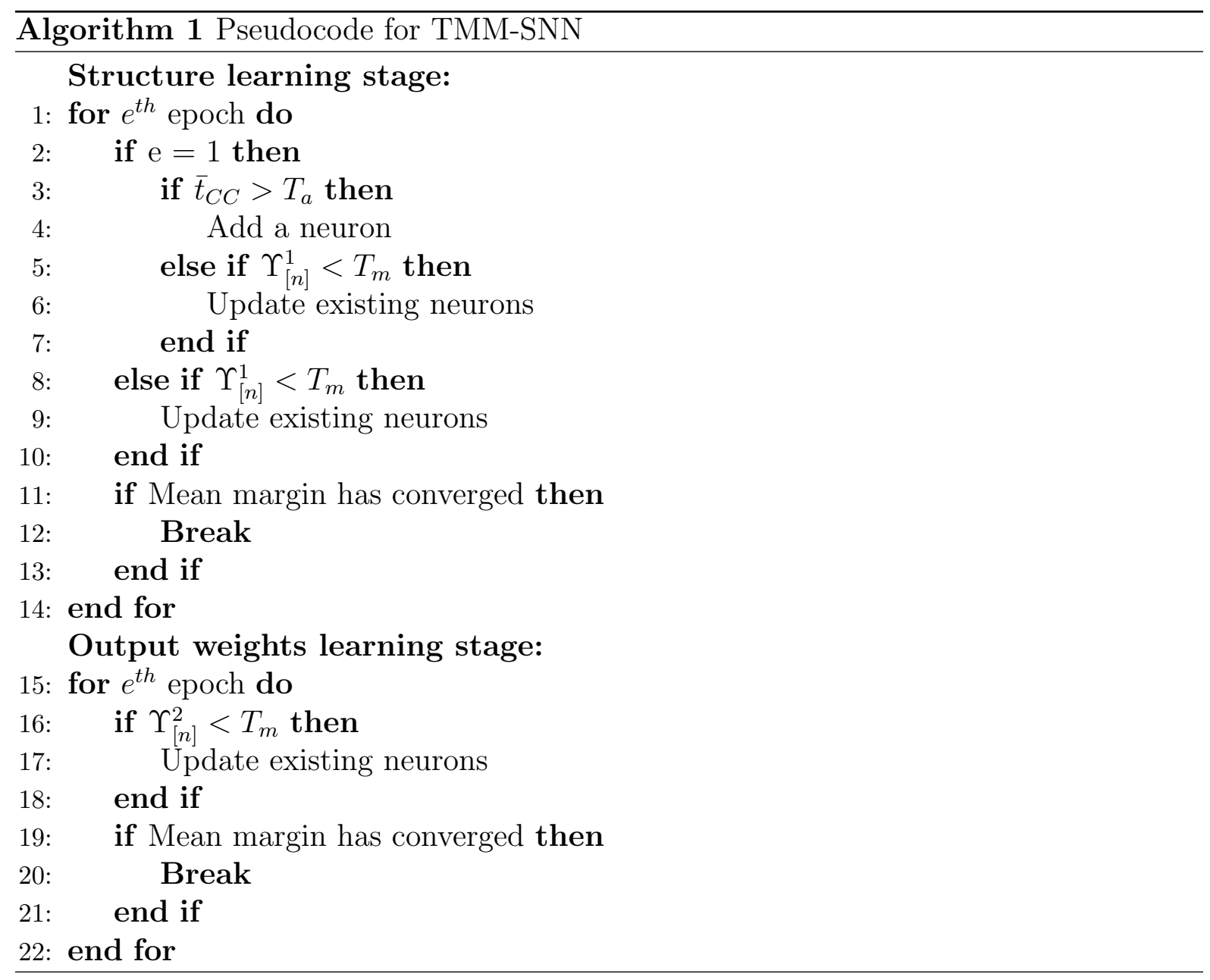

using the overall classification accuracy which is given as

$$
\text { overall accuracy }=\frac{\text { Number of correctly classified samples }}{\text { Total number of samples }} * 100
$$

In these experimental evaluations the real valued data from the Ionosphere problem is encoded into spike patterns using the population coding scheme [17] with six receptive fields per feature. The value of $T$ is set to $6 \mathrm{~ms}$ and the value of $T_{h}$ is set to $2 \mathrm{~ms}$. Next, the results of the study on the margin maximization strategy and effect of $\alpha_{a}$ on the performance of TMM-SNN are presented.

Effect of margin maximization: For this study, the value of $\alpha_{a}$ is fixed to 0.2 and the mean interclass margin $\left(\bar{\Upsilon}^{1}\right)$ for all the training samples during the structure learning 


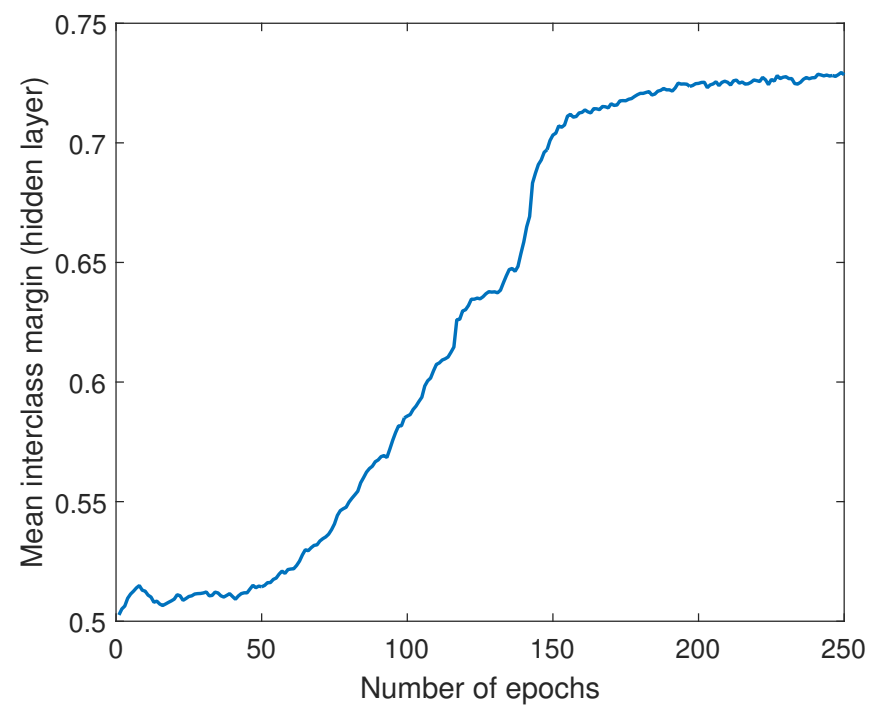

(a)

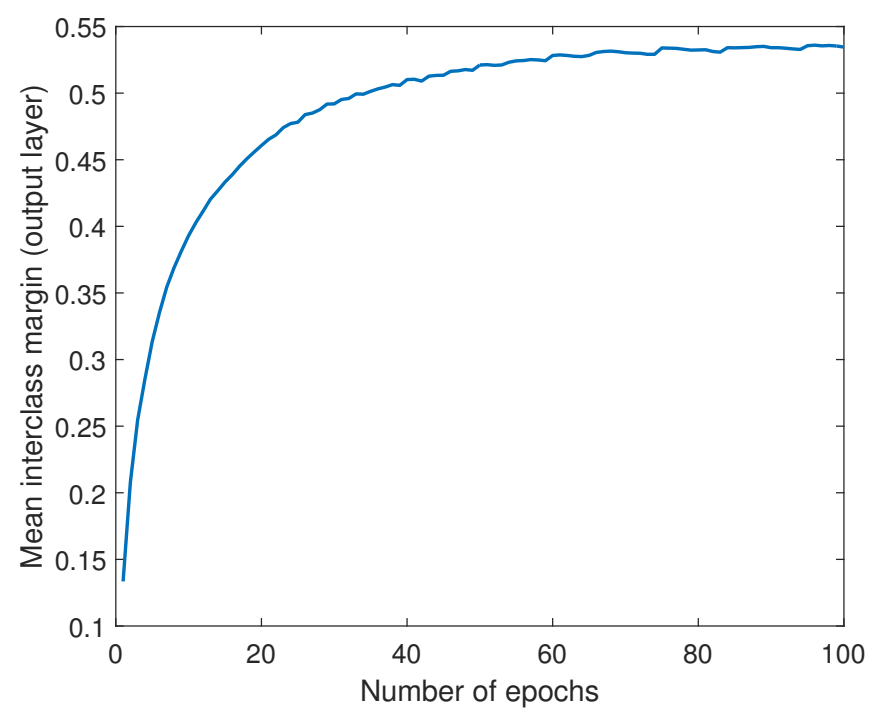

(b)

Figure 4.4: Effect of margin maximization strategy on the mean interclass margin for the response of (a) hidden layer neurons, (b) output layer neurons

stage is given by

$$
\bar{\Upsilon}^{1}=\frac{1}{N} \sum_{n} \Upsilon_{[n]}^{1}
$$

Similarly, the mean interclass margin $\left(\bar{\Upsilon}^{2}\right)$ for all the training samples during the output 


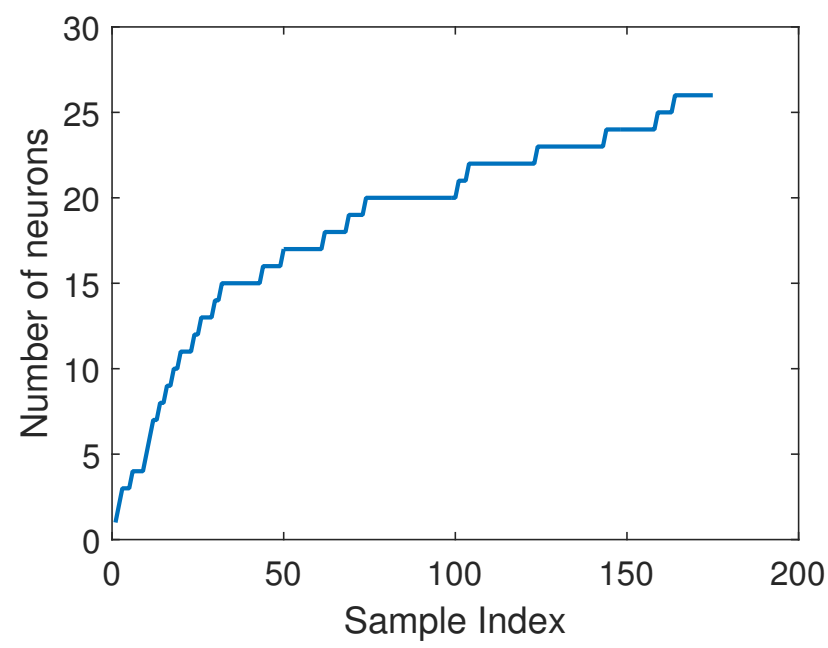

Figure 4.5: Neuron growth history of TMM-SNN for the Ionosphere problem

weights learning stage is given by

$$
\bar{\Upsilon}^{2}=\frac{1}{N} \sum_{n} \Upsilon_{[n]}^{2}
$$

Figure 4.4a shows the effect of the "margin maximization strategy for structure learning stage' on the mean interclass margin for all the training samples. It can be observed from the figure, that the mean interclass margin during the structure learning stage improves continuously until it converges to a maximum value. Figure $4.4 \mathrm{~b}$ shows the effect of 'margin maximization strategy for output weights learning stage' on the mean interclass margin for all the training samples. It can be observed from the figure that the mean interclass margin during the output weights learning stage requires fewer epochs for convergence. The fast convergence in the second stage can be attributed to the weight update in the structure learning stage. After the structure learning stage, the hidden neurons from the same class as the current input spike pattern learn to fire first for a given input spike pattern.

Effect of addition threshold: When the addition threshold $\left(\alpha_{a}\right)$ is set to 0.2 , TMM-SNN has a training and testing accuracy of $95.86 \%$ and $91.48 \%$. Figure 4.5 shows the neuron growth history for TMM-SNN. It may be noted that TMM-SNN evolves the hidden layer completely within the first epoch. It can be observed from the figure that the learning algorithm adds a higher number of neurons during the beginning of training 


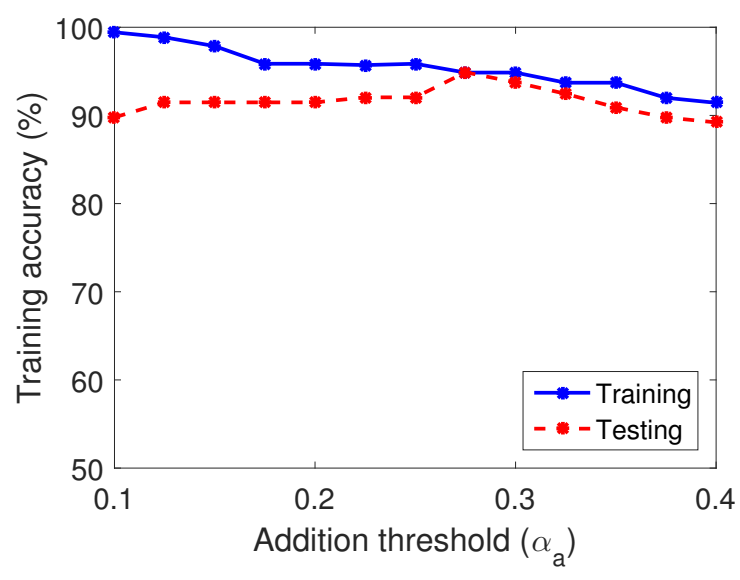

(a)

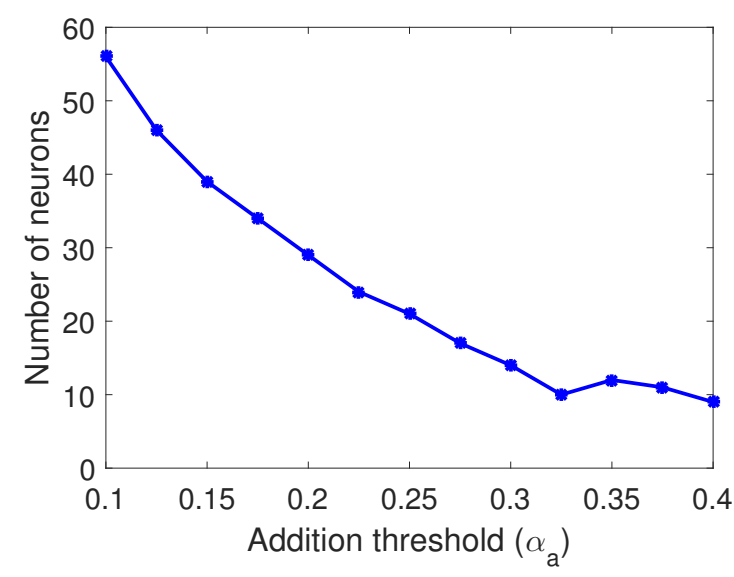

(b)

Figure 4.6: Effect of addition threshold on (a) training and testing accuracy of TMMSNN, (b) number of neurons added by TMM-SNN

and fewer neurons are added closer to the end of the training. Figure 4.6a shows the effect of $\alpha_{a}$ on the training and testing accuracy of the TMM-SNN and Figure 4.6b shows the effect of $\alpha_{a}$ on the number of neurons added by TMM-SNN during training. It can be seen from Figure 4.6a that the $\alpha_{a}$ does not affect the training and testing accuracies significantly when $\alpha_{a}$ is chosen in the interval $[0.15,0.35]$. When $\alpha_{a}$ is set to a value greater than 0.35 , both, the training and testing accuracies of TMM-SNN start deteriorating. This may be due to the addition of fewer neurons by TMM-SNN, when $\alpha_{a}$ is chosen higher than 0.35 (Figure 4.6b). When $\alpha_{a}$ is chosen lower than 0.15 , the training accuracy improves slightly and the testing accuracy goes down. This may be attributed to over-fitting because TMM-SNN adds a large number of neurons for smaller 
values of $\alpha_{a}$ (Figure 4.6b). Based on these observations, a suitable range for choosing $\alpha_{a}$ is $[0.15,0.35]$. It should be set closer to 0.15 for difficult problems with significant overlap between classes. For simpler problems, it should be set closer to 0.35 .

\subsection{Performance Evaluation of the TMM-SNN}

In this section, the performance of the Two stage Margin Maximization Spiking Neural Network is evaluated on ten benchmark classification problems from the UCI machine learning repository [21]. The results of performance evaluation have been compared with that of other existing batch learning algorithms for Spiking Neural Networks (SNNs), namely, SpikeProp [17], Synaptic Weight Association Training (SWAT) [10] and SelfRegulating Evolving Spiking Neural (SRESN) [82] classifier on all the problems. The performance evaluation results of TMM-SNN have also been compared with evolving Spiking Neural Network (eSNN) [11] and Accurate Synaptic-efficiency Adjustment (ASA) [24].

Performance results for all the algorithms (except eSNN and ASA) have been generated using MATLAB 2014b in a Windows environment on a machine with 12 logical cores, $16 \mathrm{~GB}$ memory and a speed of $3.2 \mathrm{GHz}$. All the results, reported in this section, represent the average training/testing accuracy obtained over ten random trials. The training procedure for the TMM-SNN is stopped in both the stages when the mean interclass margin for all the training samples remains constant within \pm 0.1 for 10 epochs. The mean interclass margin is computed using Equation 4.27 and 4.28, respectively in the two stages of the learning algorithm.

\subsubsection{Performance Comparison with Batch Learning Algorithms}

In this section, the performance of TMM-SNN is evaluated using ten benchmark data sets from the UCI machine learning repository [21]. Results of performance evaluation have been compared with other batch learning algorithms for SNNs, namely, SpikeProp [17], SWAT [10] and SRESN [82] classifier. Table 4.1 summarizes the details of the data sets used for this evaluation. It provides information about the number of features, classes the training/testing samples and the imbalance factor in a given data set. The imbalance 
Table 4.1: Description of the data sets used for evaluation

\begin{tabular}{|c|c|c|c|c|c|}
\hline Data set & \# Features & \# Classes & \multicolumn{2}{|c|}{ \# Samples } & $\begin{array}{c}\text { Imbalance } \\
\text { factor }(\varrho)\end{array}$ \\
\cline { 4 - 6 } & & & Training & Testing & 0.27 \\
\hline \hline Breast cancer & 9 & 2 & 350 & 333 & 0.3 \\
Echocardiogram & 10 & 2 & 66 & 65 & 0 \\
Mammogram & 9 & 2 & 80 & 11 & 0.18 \\
Liver & 6 & 2 & 170 & 175 & 0.3 \\
PIMA & 9 & 2 & 384 & 384 & 0.28 \\
Ionosphere & 34 & 2 & 175 & 176 & 0.59 \\
Hepatitis & 19 & 2 & 78 & 77 & 0 \\
\hline Iris & 4 & 3 & 75 & 75 & 0 \\
Wine & 13 & 3 & 60 & 118 & 0.1 \\
Acoustic emission & 5 & 4 & 62 & 137 & \\
\hline
\end{tabular}

factor $(\varrho)$ for a given data set is estimated as described in [93] and is given as

$$
\varrho=1-\frac{C}{\sum_{j} N_{j}} \min _{j} N_{j}
$$

where $N_{j}$ represents the number of training samples in the $j^{\text {th }}$ class.

The performance comparison has been done based on three different metrics, namely, overall training/testing accuracy, number of epochs required for convergence and the total number of network parameters to be estimated. The total number of network parameters $\left(\eta_{p}\right)$ to be estimated is given by

$$
\eta_{p}=m d_{h} K+K d_{o} C
$$

where $m$ is the number of input neurons, $K$ is the number of hidden neurons and $C$ is the total number of classes. $d_{h}$ and $d_{o}$ represent the number of delays used in the hidden layer and output layer respectively.

Results for all the algorithms have been generated using the same training/testing data splits and the mean and standard deviation for overall training/testing accuracy over ten random trials is reported. The real valued data from the data sets have been encoded into the spike patterns using the population coding [17] scheme which employs six receptive fields for encoding a single feature in all problems. The population coding used for evaluating TMM-SNN does not employ any delays which imply that a single synapse 
Table 4.2: The value for the parameter addition threshold $\left(\alpha_{a}\right)$ used for evaluating TMMSNN on the benchmark data sets

\begin{tabular}{|c|c|}
\hline Data set & $\begin{array}{c}\text { \# Novelty } \\
\text { threshold }\left(\alpha_{n}\right)\end{array}$ \\
\hline \hline Breast cancer (BC) & 0.25 \\
Echocardiogram (ECHO) & 0.35 \\
Mammogram (MAM) & 0.35 \\
Liver & 0.18 \\
PIMA & 0.18 \\
Ionosphere (ION) & 0.2 \\
Hepatitis (HEP) & 0.35 \\
\hline Iris & 0.13 \\
Wine & 0.25 \\
Acoustic emission (AE) & 0.18 \\
\hline
\end{tabular}

represents a single connection without any delay. As in the SpikeProp paper [17], the results for SpikeProp have been generated using 16 delays per synapse, a learning rate of 0.0075, a coding interval of $4 \mathrm{~ms}$ and a time constant of $7 \mathrm{~ms}$. The appropriate number of hidden neurons for SpikeProp have been determined using the constructive-destructive procedure [85]. For SWAT, the parameters for the neuron model and frequency filtering are set as in [10]. Other significant parameters for SWAT are $c_{0}$ and the height of the plasticity window $\left(A_{p}\right)$ in spike timing dependent plasticity. As mentioned in the above paper, the value for $c_{0}$ is fixed at 4000 and $A_{p}$ is fixed at 0.1 to avoid oscillations in the learning process. In case of TMM-SNN, $T$ and $T_{h}$ are set to $6 \mathrm{~ms}$ and $2 \mathrm{~ms}$ respectively. The value for addition threshold is determined using ten-fold cross validation. Table 4.2 shows the value for the addition threshold $\left(\alpha_{a}\right)$ used for TMM-SNN on the benchmark data sets.

Table 4.3 shows the average execution times per epoch for TMM-SNN on a single trial of all the data sets used for evaluation. Table 4.4 shows the architecture and the results of performance evaluation for TMM-SNN, SpikeProp, SWAT and the SRESN classifier. The architecture has been shown in the format $(m-H-N)$. For the SRESN classifier and TMM-SNN the number of hidden neurons in the architecture as well as the number of network parameters have been shown as a range for the ten random trials. Further, the architecture for the SRESN classifier are shown in the format $(m-H)$ as it employs a two layered SNN. 
Table 4.3: Average execution per epoch of the SRESN classifier

\begin{tabular}{|c|c|c|}
\hline \multirow[t]{2}{*}{ Data set } & \multicolumn{2}{|c|}{ Execution time per epoch (seconds) } \\
\hline & $\begin{array}{c}\text { Structure learning } \\
\text { stage }\end{array}$ & $\begin{array}{l}\text { Output weights } \\
\text { learning stage }\end{array}$ \\
\hline Breast cancer & 2.27 & 2.66 \\
\hline Echocardiogram & 0.36 & 0.36 \\
\hline Mammogram & 0.33 & 0.35 \\
\hline Liver & 0.97 & 1.03 \\
\hline PIMA & 2.77 & 3.09 \\
\hline Ionosphere & 5.5 & 4.25 \\
\hline Hepatitis & 0.53 & 0.51 \\
\hline Iris & 0.25 & 0.25 \\
\hline Wine & 0.38 & 0.4 \\
\hline Acoustic Emission & 0.23 & 0.25 \\
\hline
\end{tabular}

From the Table 4.4, it may be observed that for simple binary classification problems like breast cancer and Echocardiogram, the training as well as the testing accuracy of TMM-SNN is similar to that of other algorithms. For the breast cancer problem, TMMSNN requires only 70 epochs for convergence whereas among other algorithms, the SRESN classifier requires a minimum of 306 epochs. For the Echocardiogram problem, TMMSNN requires 177 epochs compared to 476 epochs required by the SRESN classifier.

For a low dimensional binary classification problem with a high interclass overlap like Mammogram and Liver, the training performance of TMM-SNN is 3\%-5\% better than that of SpikeProp which is the next best performing algorithm. A similar trend is also observed for the testing accuracy of TMM-SNN which also show an improvement of 3\%-5\% over the testing accuracy of SpikeProp. Further, TMM-SNN converged in 176 and 442 epochs, respectively whereas SpikeProp was trained for 1000 epochs. PIMA is another low dimensional binary classification problem with a high interclass overlap. In case of PIMA, the training and testing accuracies of TMM-SNN are $1 \%$ and $2 \%$ better than that of SpikeProp. For this problem, TMM-SNN requires only 160 epochs whereas SpikeProp requires 3000 epochs. It may be noted, that for all these problems TMM-SNN requires lower than one-tenth the number of network parameters required by SpikeProp.

The performance of TMM-SNN has also been evaluated for high dimensional problems of Ionosphere and Hepatitis. For Ionosphere, the training and testing accuracies of TMMSNN are $7 \%$ and $4 \%$ better than that of SRESN, which is the next best performing 
Table 4.4: Performance comparison of TMM-SNN with SpikeProp, SWAT and SRESN for benchmark problems

\begin{tabular}{|c|c|c|c|c|c|c|}
\hline $\begin{array}{l}\text { Data } \\
\text { set }\end{array}$ & $\begin{array}{c}\text { Learning } \\
\text { Algorithm }\end{array}$ & Architecture & $\begin{array}{c}\text { Training } \\
\text { Accuracy (\%) }\end{array}$ & $\begin{array}{c}\text { Testing } \\
\text { Accuracy }(\%)\end{array}$ & $\begin{array}{c}\# \\
\text { Epochs }\end{array}$ & $\begin{array}{l}\text { \# Network } \\
\text { Parameters }\end{array}$ \\
\hline \multirow{4}{*}{$\mathrm{BC}$} & SpikeProp & $55-15-2$ & $97.3(0.6)$ & $97.2(0.6)$ & 1000 & 13680 \\
\hline & SWAT & $54-702-2$ & $96.5(0.5)$ & $95.8(1.0)$ & 500 & 1404 \\
\hline & SRESN & $54-(8-12)$ & $97.7(0.6)$ & $97.2(0.7)$ & 306 & $(432-648)$ \\
\hline & TMM-SNN & $54-(2-8)-2$ & $97.4(0.3)$ & $97.2(0.5)$ & 70 & $(112-448)$ \\
\hline \multirow{4}{*}{$\mathrm{ECHO}$} & SpikeProp & $61-10-2$ & $86.6(2.5)$ & $84.5(3.0)$ & 1000 & 10080 \\
\hline & SWAT & $60-780-2$ & $90.6(1.8)$ & $81.8(2.8)$ & 500 & 1560 \\
\hline & SRESN & $60-(2-4)$ & $79.1(5.7)$ & $77.7(5.3)$ & 476 & $(120-240)$ \\
\hline & TMM-SNN & $60-(2-3)-2$ & $86.5(2.1)$ & $85.4(1.7)$ & 177 & $(124-186)$ \\
\hline \multirow{4}{*}{ MAM } & SpikeProp & $55-10-2$ & $82.8(4.7)$ & $81.8(6.1)$ & 1000 & 9120 \\
\hline & SWAT & $54-702-2$ & $82.6(2.1)$ & $78.2(12.3)$ & 500 & 1404 \\
\hline & SRESN & $54-(4-8)$ & $75.0(2.8)$ & $76.6(4.5)$ & 86 & $(216-432)$ \\
\hline & TMM-SNN & $54-(5-7)-2$ & $87.2(4.4)$ & $84.9(8.6)$ & 176 & $(280-392)$ \\
\hline \multirow{4}{*}{ Liver } & SpikeProp & $37-15-2$ & $71.5(5.2)$ & $65.1(4.7)$ & 3000 & 9360 \\
\hline & SWAT & $36-468-2$ & $74.8(2.1)$ & $60.9(3.2)$ & 500 & 936 \\
\hline & SRESN & $36-(6-9)$ & $60.4(1.7)$ & $59.7(1.7)$ & 715 & $(216-324)$ \\
\hline & TMM-SNN & $36-(5-8)-2$ & $74.2(3.5)$ & $70.4(2.0)$ & 442 & (190-304) \\
\hline \multirow{4}{*}{ PIMA } & SpikeProp & $55-20-2$ & $78.6(2.5)$ & $76.2(1.8)$ & 3000 & 16640 \\
\hline & SWAT & $54-702-2$ & $77.0(2.1)$ & $72.1(1.8)$ & 500 & 1404 \\
\hline & SRESN & $54-(9-14)$ & $70.5(2.4)$ & $69.9(2.1)$ & 254 & $(486-756)$ \\
\hline & TMM-SNN & $54-(5-14)-2$ & $79.7(2.3)$ & $78.1(1.7)$ & 160 & $(280-784)$ \\
\hline \multirow{4}{*}{ ION } & SpikeProp & $205-25-2$ & $89.0(7.9)$ & $86.5(7.2)$ & 3000 & 82800 \\
\hline & SWAT & $204-2652-2$ & $86.5(6.7)$ & $90.0(2.3)$ & 500 & 5304 \\
\hline & SRESN & $204-(16-23)$ & $91.9(1.8)$ & $88.6(1.6)$ & 1018 & $(3264-4692)$ \\
\hline & TMM-SNN & $204-(23-34)-2$ & $98.7(0.4)$ & $92.4(1.8)$ & 246 & $(4738-7004)$ \\
\hline \multirow{4}{*}{ HEP } & SpikeProp & $115-15-2$ & $87.8(5.0)$ & $83.5(2.5)$ & 1000 & 28080 \\
\hline & SWAT & $114-1482-2$ & $86.0(2.1)$ & $83.1(2.2)$ & 500 & 2964 \\
\hline & SRESN & 114-(4-10) & $79.8(0.9)$ & $78.5(1.8)$ & 234 & $(456-1140)$ \\
\hline & TMM-SNN & 114-(3-9)-2 & $91.2(2.5)$ & $86.6(2.2)$ & 192 & $(348-1044)$ \\
\hline \multirow{4}{*}{ Iris } & SpikeProp & $25-10-3$ & $97.2(1.9)$ & $96.7(1.6)$ & 1000 & 4480 \\
\hline & SWAT & $24-312-3$ & $96.7(1.4)$ & $92.4(1.7)$ & 500 & 936 \\
\hline & SRESN & $24-(6-10)$ & $96.9(1.0)$ & $97.3(1.3)$ & 102 & $(144-240)$ \\
\hline & TMM-SNN & $24-(4-7)-3$ & $97.5(0.8)$ & $97.2(1.0)$ & 94 & $(108-189)$ \\
\hline \multirow{4}{*}{ Wine } & SpikeProp & $79-10-2$ & $99.2(1.2)$ & $96.8(1.6)$ & 1000 & 12960 \\
\hline & SWAT & 78-1014-3 & $98.6(1.1)$ & $92.3(2.4)$ & 500 & 2028 \\
\hline & SRESN & $78-(5-10)$ & $96.9(1.6)$ & $91.0(1.2)$ & 128 & $(390-780)$ \\
\hline & TMM-SNN & $78-3-3$ & $100(0)$ & $97.5(0.8)$ & 80 & 243 \\
\hline \multirow{4}{*}{$\mathrm{AE}$} & SpikeProp & $31-10-4$ & $98.5(1.7)$ & $97.2(3.5)$ & 1000 & 5600 \\
\hline & SWAT & $30-390-4$ & $93.1(2.3)$ & $91.5(2.3)$ & 500 & 1560 \\
\hline & SRESN & $30-(4-8)$ & $93.9(5.9)$ & $94.2(3.2)$ & 633 & $(120-240)$ \\
\hline & TMM-SNN & $30-(4-7)-4$ & $97.6(1.3)$ & $97.5(0.7)$ & 12 & $(136-238)$ \\
\hline
\end{tabular}

algorithm. TMM-SNN and SRESN required 246 and 1018 epochs, respectively to achieve the reported accuracy. It may be observed that among other algorithms SWAT had the best testing accuracy, but its performance results were inconsistent as its training 
accuracy is lower than its testing accuracy. For the Hepatitis problem, the training and testing accuracies of TMM-SNN are $4 \%$ and 3\% better than that of SpikeProp, respectively. The reported accuracy is achieved by SpikeProp in 1000 epochs, whereas the TMM-SNN requires only 192 epochs.

For multiclass classification problems, the performance of TMM-SNN is similar to the performance of SpikeProp. In comparison to SWAT and SRESN, the training accuracy of TMM-SNN is $4 \%$ better and the testing accuracy is $6 \%$ and $3 \%$ better, respectively. With regards to the number of epochs, TMM-SNN requires much lesser epochs to achieve convergence in comparison to the other algorithms used for comparison. Further, TMMSNN achieves a much compact network architecture in comparison to the other algorithms used for comparison.

Statistical analysis of performance comparison: The results of performance evaluation of the learning algorithms SpikeProp, SWAT, SRESN and TMM-SNN are also been compared using the one-way analysis of variance (ANOVA) test [22] followed by a pairwise comparison using the Fisher's least significant difference method [23]. The one-way ANOVA test was conducted with the null hypothesis that the performance of the different learning algorithms do not differ significantly from one another. If the $p$-value for the computed statistic is lesser than 0.05 than the null hypothesis is rejected within a $95 \%$ confidence interval. The ANOVA test is conducted using the mean testing accuracies on the ten data sets described above. A $p$-value of 3e- 6 is obtained which implies that the null hypothesis can be rejected within a $95 \%$ confidence interval. This shows that not all the algorithms perform equally. For further analysis, a pairwise test was conducted using the Fisher's Least Significant Difference (LSD) method. Using the LSD method, the $p$-values obtained for the pairwise comparisons of TMM-SNN with SpikeProp, SWAT and SRESN are 0.0248, 1e-5 and 1e-6, respectively. Since, all the $p$-values are lesser than 0.05, TMM-SNN performs better than the other algorithm used for comparison within a $95 \%$ confidence interval.

These observations clearly highlight that the margin based approach for updating the network parameters helps TMM-SNN achieve a better or similar generalization performance in comparison to existing learning algorithms for spiking neural networks using a compact network structure. The margin based approach in TMM-SNN has been specif- 
ically developed to handle pattern classification problems. Most of the other existing learning algorithms for SNNs update the synaptic weights based on the difference between the actual and the desired output spike pattern. This approach does not take into account the overlap between classes while updating the synaptic weights. To overcome this problem TMM-SNN updates the synaptic weights to maximize the interclass margin. Further, the use of activation based coding to initialize the weights of the hidden layer neurons during the structure learning stage helps TMM-SNN converge faster in comparison to other existing learning algorithms for SNNs.

\subsubsection{Performance Comparison with Evolving Spiking Neural Network}

In this section, the performance of the TMM-SNN is compared with the performance of the evolving Spiking Neural Network (eSNN) [11] for five benchmark data sets from the UCI machine learning repository [21]. For this purpose, the number of training and testing samples used for evaluating the TMM-SNN are same as those used for evaluating eSNN in [61]. The details of the data sets used for comparison has been provided in Table 4.5. The results for TMM-SNN have been generated using five random trials but for eSNN, the number of trials have not been reported in the paper. The mean and standard deviation for the performance of TMM-SNN for ten random trials have also been reported. The results for eSNN have been reproduced from [61]. The results of TMMSNN have been generated using the same population coding parameters as described in the previous section.

Table 4.5: Description of the benchmark data set used for the comparison between eSNN and TMM-SNN

\begin{tabular}{|c|c|c|c|c|}
\hline Data set & \# Features & \# Classes & \multicolumn{2}{|c|}{ \# Samples } \\
\cline { 3 - 5 } & & & Training & Testing \\
\hline Breast cancer (BC) & 9 & 2 & 455 & 228 \\
Iris & 4 & 3 & 90 & 60 \\
Liver & 6 & 2 & 230 & 115 \\
PIMA & 9 & 2 & 512 & 256 \\
Ionosphere (Ion) & 34 & 2 & 234 & 117 \\
\hline
\end{tabular}


Table 4.6: Performance comparison of TMM-SNN and eSNN

\begin{tabular}{|c|c|c|c|c|c|c|}
\hline \multirow{2}{*}{$\begin{array}{c}\text { Data } \\
\text { set }\end{array}$} & \multicolumn{3}{|c|}{ eSNN } & \multicolumn{3}{|c|}{ TMM-SNN } \\
\cline { 2 - 7 } & $\begin{array}{c}\text { Training } \\
\text { Accuracy (\%) }\end{array}$ & $\begin{array}{c}\text { Testing } \\
\text { Accuracy (\%) }\end{array}$ & \# Neurons & $\begin{array}{c}\text { Training } \\
\text { Accuracy (\%) }\end{array}$ & $\begin{array}{c}\text { Testing } \\
\text { Accuracy (\%) }\end{array}$ & $\begin{array}{c}\# \\
\text { Neurons }\end{array}$ \\
\hline BC & $\mathbf{9 9 . 6}$ & $\mathbf{9 8 . 7}$ & 280 & $97.5(0.4)$ & $97.8(1.0)$ & $3-6$ \\
Iris & $\mathbf{1 0 0}$ & 95 & 84 & $98.5(0.9)$ & $\mathbf{9 8 . 0 ( 0 . 7 )}$ & $4-6$ \\
Liver & $\mathbf{8 6 . 5}$ & 59.1 & 215 & $74.4(2.5)$ & $\mathbf{6 7 . 7}(\mathbf{0 . 9})$ & $7-9$ \\
PIMA & $\mathbf{8 1}$ & 61.7 & 265 & $80.9(1.1)$ & $\mathbf{7 8 . 6 ( 1 . 3 )}$ & $12-13$ \\
ION & 81.6 & 74.4 & 213 & $\mathbf{9 8 . 6 ( 0 . 4 )}$ & $\mathbf{9 3 . 2 ( 1 . 2 )}$ & 20 \\
\hline
\end{tabular}

Table 4.6 shows the results of performance comparison between eSNN and TMMSNN. The table shows the mean training/testing accuracy and standard deviation of the performance of TMM-SNN for five random trials. The table also shows the number of neurons used by each learning algorithm.

It can be observed from the table that for simple binary classification problems like BC, TMM-SNN performs almost at par with eSNN. For simple multiclass problems like Iris, TMM-SNN performs $3 \%$ better than eSNN. For difficult problems like Liver, PIMA and Ionosphere, TMM-SNN performs 8-19\% better than eSNN. For all the data sets, TMM-SNN employs a very compact network architecture in comparison to eSNN.

The results of performance comparison clearly highlight that TMM-SNN performs significantly better than eSNN for all the data sets used in comparison. The improvement in performance can be attributed to the margin maximization based learning approach employed by TMM-SNN.

\subsubsection{Performance Comparison with Accurate Synaptic-Efficiency Adjustment}

In this section, the performance of the TMM-SNN is compared with the performance of the Accurate Synaptic-efficiency Adjustment (ASA) [24] method for five benchmark data sets from the UCI machine learning repository [21]. For this purpose, the number of training and testing samples used for evaluating the TMM-SNN are same as those used for evaluating ASA in [24]. The details of the data sets used for comparison has been provided in Table 4.7. The results for both ASA and TMM-SNN have been generated using ten random trials. The mean and standard deviation of overall accuracy for the 
Table 4.7: Description of the benchmark data set used for the comparison between ASA and TMM-SNN

\begin{tabular}{|c|c|c|c|c|}
\hline Data set & \# Features & \# Classes & \multicolumn{2}{|c|}{ \# Samples } \\
\cline { 4 - 5 } & & & Training & Testing \\
\hline Breast cancer (BC) & 9 & 2 & 614 & 69 \\
Liver & 6 & 2 & 310 & 35 \\
PIMA & 9 & 2 & 691 & 77 \\
Iris & 4 & 3 & 135 & 15 \\
Glass identification (GI) & 9 & 6 & 192 & 22 \\
\hline
\end{tabular}

ten random trials is reported. The results for ASA have been reproduced from [24]. The results of TMM-SNN have been generated using the same population coding parameters as used for evaluating ASA in [24]. The number of receptive fields is fixed at 12 and the overlap constant is set to 1.5 .

Table 4.8 shows the results of performance comparison between ASA and TMM-SNN. The table shows the mean training and testing accuracy and the mean number of epochs required for convergence for the ten random trials of the two learning algorithms, ASA and TMM-SNN.

For the simple binary classification problem of breast cancer, TMM-SNN performs $3 \%$ better than ASA. TMM-SNN requires 111 epochs for convergence whereas ASA requires only 2 epochs. For difficult binary classification problems of Liver and PIMA, the performance of TMM-SNN is 4-11\% better than ASA. TMM-SNN requires 508 and 434 epochs to achieve the reported accuracy for the Liver and PIMA problems respectively. ASA achieves the reported accuracy using 3 and 2 epochs for the Liver and PIMA problem respectively.

Table 4.8: Performance comparison of TMM-SN and ASA

\begin{tabular}{|c|c|c|c|c|c|c|}
\hline \multirow{2}{*}{$\begin{array}{c}\text { Data } \\
\text { set }\end{array}$} & \multicolumn{3}{|c|}{ ASA } & \multicolumn{3}{|c|}{ TMM-SNN } \\
\cline { 2 - 6 } & $\begin{array}{c}\text { Training } \\
\text { Accuracy (\%) }\end{array}$ & $\begin{array}{c}\text { Testing } \\
\text { Accuracy (\%) }\end{array}$ & \# Epochs & $\begin{array}{c}\text { Training } \\
\text { Accuracy (\%) }\end{array}$ & $\begin{array}{c}\text { Testing } \\
\text { Accuracy (\%) }\end{array}$ & \# Epochs \\
\hline BC & 96 & 95 & 2 & $\mathbf{9 7 . 9 ( 0 . 2 )}$ & $\mathbf{9 8 . 1 ( 1 . 7 )}$ & 111 \\
Liver & 76 & 60 & 3 & $\mathbf{8 0 . 6 ( 3 . 8 )}$ & $\mathbf{7 1 . 7}(\mathbf{4 . 3 )}$ & 508 \\
PIMA & 77 & 72 & 2 & $\mathbf{8 2 . 2 ( 1 . 5 )}$ & $\mathbf{7 6 . 1 ( 3 . 9 )}$ & 434 \\
Iris & 96 & 95 & 2 & $\mathbf{9 8 . 3 ( 0 . 5 )}$ & $\mathbf{9 9 . 3 ( 2 . 0 )}$ & 97 \\
GI & 85 & 76 & - & $\mathbf{8 7 . 5 ( 3 . 0 )}$ & $\mathbf{8 0 . 5 ( 2 . 9 )}$ & 480 \\
\hline
\end{tabular}


The results for ASA and TMM-SNN have also been compared using the multi-class problems of Iris flower classification and Glass identification. For the simple Iris problem TMM-SNN performs $4 \%$ better than ASA. With regards to the number of epochs, ASA and TMM-SNN require 2 and 97 epochs, respectively for the Iris problem. For the difficult Glass problem, the performance of TMM-SNN is $4 \%$ better than ASA and it requires 480 epochs for convergence. The number of epochs required by ASA has not been reported in $[24]$.

The results of performance comparison clearly highlight that the TMM-SNN can closely approximate the relationship between the input spike patterns and the corresponding class labels. Further, the maximization of the interclass margin helps TMM-SNN in achieving better generalization performance in comparison to ASA.

\subsection{Summary}

In this chapter, a Two stage Margin Maximization Spiking Neural Network (TMM-SNN) has been presented. TMM employs a three layered SNN. The learning algorithm for TMM-SNN employs a two stage learning algorithm to evolve the network architecture and estimate the network parameters. In the first stage (structure learning stage), the learning algorithm evolves the hidden layer neurons and estimates the synaptic weights and thresholds for the hidden layer neurons. In this stage, the learning algorithm uses two different strategies, viz. 'neuron addition strategy' and the 'margin maximization strategy for structure learning'. The 'neuron addition strategy' is used by the learning algorithm to evolve the hidden layer completely in a single epoch. The weights for the hidden layer neurons are initialized using the new activation based coding scheme, developed in this thesis. The 'margin maximization strategy for structure learning' is used by the learning algorithm for multiple epochs to maximize the separation between the spike times of interclass neurons in the hidden layer. After the structure learning stage, the synaptic weights and thresholds for the hidden layer neurons are kept fixed. In the second stage, the learning algorithm estimates the synaptic weights for the output layer neurons. The learning algorithm uses the 'margin maximization strategy for output weights learning' for multiple epochs to maximize the separation between the spike times of the interclass 
neurons in the output layer.

The performance of the learning algorithm is evaluated on a set of benchmark data sets from the UCI machine learning repository and is compared with the performance of other existing learning algorithms for spiking neural networks. The results of performance evaluation clearly indicate that TMM-SNN can achieve better performance using a compact network. Also, TMM-SNN requires fewer epochs in comparison to other algorithms except eSNN and ASA. Thus, TMM-SNN is able to closely approximate the relationship between the input spike patterns and the corresponding class labels. But the margin based learning approach of TMM-SNN makes it more suitable for classification problems. It may not be able to achieve superior performance for other problems like regression, etc. Further, the learning algorithm utilizes only the locally available information for updating the synaptic weights of the neurons in the network. This renders the learning algorithm unsuitable for learning in an online framework. To overcome these limitations, a meta-neuron based learning rule for a spiking neural network with a meta-neuron is developed in the next chapter. 
Chapter 4. TMM-SNN 


\section{Chapter 5}

\section{An Online Meta-neuron based Learning Algorithm}

In the previous chapter, a Two stage Margin Maximization Spiking Neural Network (TMM-SNN) was developed. The learning algorithm for TMM-SNN utilized locally available information to initialize and update the weights of neurons in the network. But, the local learning strategies in TMM-SNN render it unsuitable for learning in an online learning framework. To learn effectively in an online framework, a learning algorithm needs to consider the global knowledge stored in the network while updating the network parameters. Thus, there is need to develop a heterosynaptic plasticity mechanism for spiking neural networks; that is capable of updating the network parameters in an online manner.

It has been shown in recent neuroscience literature that the astrocyte cells in the brain are capable of exhibiting a heterosynaptic plasticity mechanism to update the synaptic weights of a neuron in the brain [86]. The astrocyte cells are capable of connecting to a large number of synapses simultaneously [87, 88], which allows them to intercept and modulate the activity on these synapses. Such a heterosynaptic plasticity mechanism realized by astrocytes enables them to take into account the global information present in the network while updating the weights of a given synapse.

Inspired by the heterosynaptic plasticity mechanism exhibited by astrocytes, the concept of meta-neuron is developed in this chapter. The meta-neuron has access to the global knowledge stored in the network and the local information present in the input spike patterns. Based on the local and global information available to the meta-neuron, it 
estimates the weight sensitivity modulation factor for the synaptic connections between the input and output neurons. Using the weight sensitivity modulation factor for a given synapse and the postsynaptic potential, the meta-neuron based learning rule adjusts the synaptic weights in the network to produce precise shifts in the spike times of the postsynaptic neurons. The ability of the meta-neuron based learning rule to produce precise shifts in the spike times of the postsynaptic neurons renders it suitable for learning in an online manner. To demonstrate this capability of the meta-neuron based learning rule, an online meta-neuron based learning algorithm has been developed for pattern classification problems. The Online Meta-neuron based Learning Algorithm (OMLA) employs three different heuristic learning strategies to evolve and update the network parameters. Depending on the information present in the current input spike pattern and the knowledge stored in the network, OMLA chooses to either add a neuron or delete the spike pattern or update the network parameters.

The performance of the OMLA has been evaluated on a set of benchmark problems from the UCI machine learning repository [21]. The results of performance evaluation of OMLA have been compared with existing online learning algorithms for spiking neural networks. The results of performance evaluation have been compared using the ANOVA statistical test and it can be clearly seen that OMLA achieves better performance in comparison to the existing online learning algorithms for spiking neural networks. For the sake of completeness, the performance of OMLA has also been compared with the performance of existing batch learning algorithms for spiking neural networks. It can be clearly observed from the results of performance evaluation that OMLA achieves better performance than existing batch learning algorithms for spiking neural networks. Also, OMLA requires a single epoch for achieving the reported results whereas other batch learning algorithms require multiple epochs for convergence. Next, the meta-neuron based learning rule will be described followed by a detailed description of the online meta-neuron based learning algorithm. 


\subsection{Spiking Neural Network with a Meta-neuron}

In this section, the meta-neuron based learning rule is developed for updating the synaptic weights of a two layered spiking neural network with a meta-neuron. The concept of metaneuron is inspired by the heterosynaptic plasticity induced by astrocyte cells in biological neural networks $[86,89,90]$. It has been shown in literature, that astrocyte cells can be simultaneously connected to multiple synapses $[87,88]$ which allows them to intercept the activities on the connected synapses and modulate the plasticity of these synapses. Such a heterosynaptic plasticity mechanism is a good candidate for utilization of global information stored in the network while updating the synaptic weights in the network.

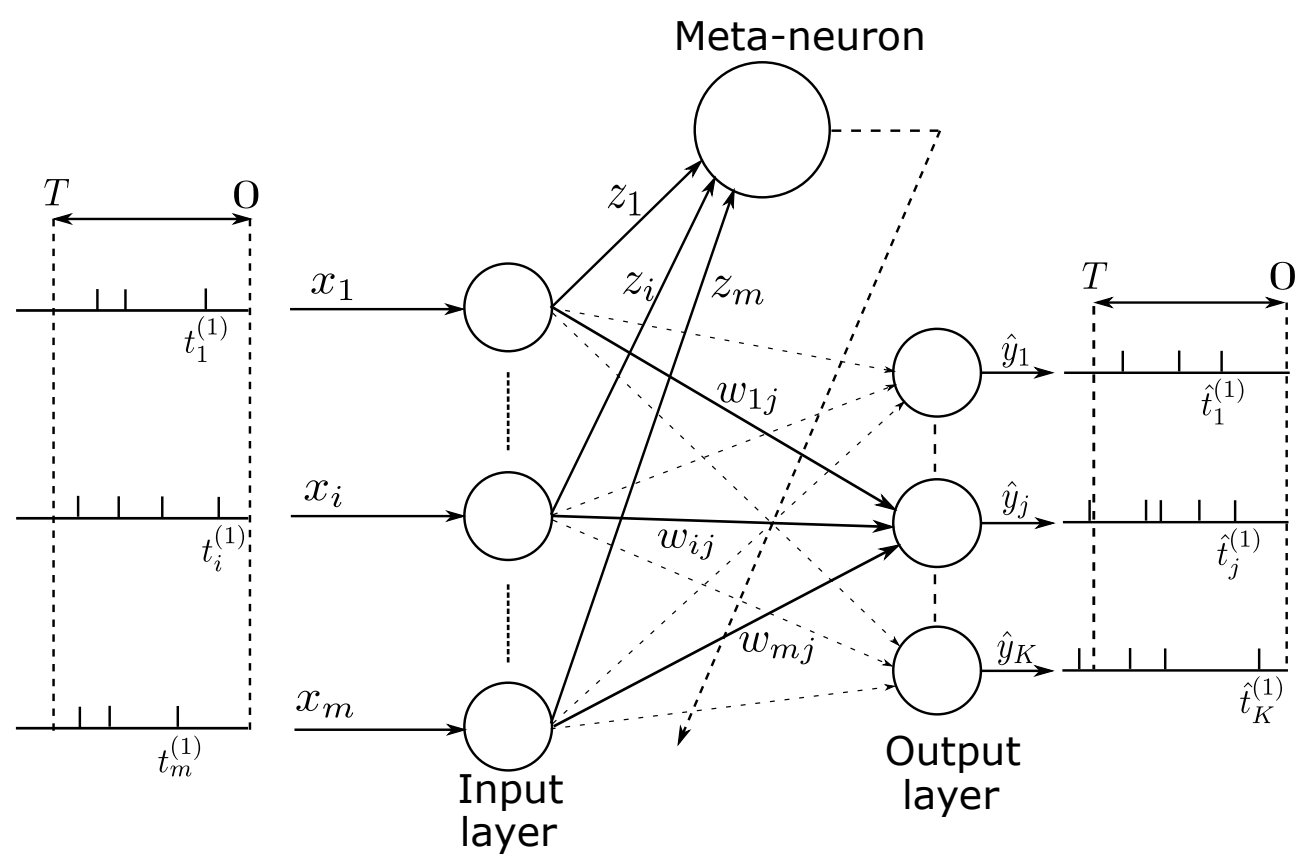

Figure 5.1: Architecture of a two layered spiking neural network with a meta-neuron. The input and output layer consists of $m$ and $n$ spiking neurons, respectively. The network is fully connected between the input and output layer. Further, the input layer neurons are also connected to the meta-neuron, which provides the meta-neuron access to the local information present in the input spike train on a particular synapse. The meta-neuron also has access to the global information (synaptic weights) stored in the network, represented by the oblique arrow from the meta-neuron across the network.

Inspired by this behavior of astrocytes, in this thesis, we develop a meta-neuron based learning rule for a spiking neural network with the meta-neuron. Figure 5.1 shows the architecture of the two layered spiking neural network with the meta-neuron. The 
network is presented with spike patterns at intervals of time $T$, where $T$ is termed as the simulation interval for a single spike pattern. For a given $m$-dimensional input spike pattern $\mathbf{x}=\left[x_{1}, \cdots, x_{i}, \cdots, x_{m}\right]^{T}, \mathbf{y}=\left[y_{1}, \cdots, y_{j}, \cdots, y_{n}\right]^{T}$ is the desired $n$-dimensional output spike pattern and $\hat{\mathbf{y}}=\left[\hat{y}_{1}, \cdots, \hat{y}_{j}, \cdots, \hat{y}_{n}\right]^{T}$ is the actual spike pattern generated by the network. Here, $x_{i}=\left\{t_{i}^{(1)}, \cdots, t_{i}^{(g)}, \cdots, t_{i}^{\left(G_{i}\right)}\right\}$ is a spike pattern with $G_{i}$ spikes, where $t_{i}^{(g)} \in[0, T]$ represents the $g^{t h}$ spike generated by the $i^{\text {th }}$ input neuron. Similarly, $y_{j}=$ $\left\{t_{j}^{(1)}, \cdots, t_{j}^{(g)}, \cdots, t_{j}^{\left(G_{j}\right)}\right\}$ and $\hat{y}_{j}=\left\{\hat{t}_{j}^{(1)}, \cdots, \hat{t}_{j}^{(g)}, \cdots, \hat{t}_{j}^{\left(G_{j}\right)}\right\}$ are spike patterns with $G_{j}$ spikes. The network is fully connected and the weight of the synapse between the $i^{\text {th }}$ input neuron and the $j^{\text {th }}$ output neuron is represented by $w_{i j}$. Further, all the input neurons are also connected to the meta-neuron through synapses with weights $\mathbf{z}=\left[z_{1}, \cdots, z_{i}, \cdots, z_{m}\right]$. Also, the meta-neuron has access to the weights of the output neurons (shown by the oblique arrow in the figure).

The meta-neuron is a typical spiking neuron having access to the local information in the spike pattern generated by a given input neuron and the global information stored in the network (synaptic weights of output neurons). It uses this information to compute its weights $(\mathbf{z})$ while updating the weights of a given output neuron and determines the weight sensitivity modulation factor for each synapse of that output neuron. The metaneuron based learning rule uses the weight sensitivity modulation factor and the required change in the postsynaptic potential of a given output neuron to produce precise shifts in its spike times by updating its synaptic weights.

The objective of the learning rule is to closely capture the functional relationship between the input spike patterns and the desired output spike patterns. For this purpose, the learning rule is used to update the synaptic weights for each postsynaptic spike individually. For the sake of brevity, the following discussion describes the learning rule for the $g^{t h}$ spike generated by the $j^{\text {th }}$ output neuron. The error $\left(e_{j}^{(g)}\right)$ in the spike time of the $g^{\text {th }}$ spike generated by the $j^{\text {th }}$ neuron is given as

$$
e_{j}^{(g)}=\hat{t}_{j}^{(g)}-t_{j}^{(g)}
$$

It may be noted that, during training, the number of desired and actual spikes generated by the output neurons may not be equal. In case the number of actual spikes generated by the $j^{\text {th }}$ output neuron is less than $G_{j}$, then the actual spike times for the missing 
spikes are set to $T+\delta$. Here, $\delta$ is a small positive number and a spike time of $(T+\delta)$ implies an absence of spike in the simulation interval. When the number of actual spikes generated by the $j^{\text {th }}$ output neuron is greater than $G_{j}$, then the desired spike times for the extra spikes are set to $T+\delta$.

In order to minimize the differences between the actual and desired spike times, the synaptic weights of the $j^{\text {th }}$ output neuron are updated such that its actual spike times are shifted in the direction of the corresponding desired spike times. Suppose, $\bar{t}_{j}^{(g)}$ is the spike time for the $g^{\text {th }}$ spike generated by the $j^{\text {th }}$ neuron after updating its weights, then $\bar{t}_{j}^{(g)}$ is given as

$$
\bar{t}_{j}^{(g)}=\hat{t}_{j}^{(g)}-\eta\left(e_{j}^{(g)}\right), \quad j \in\{1, \cdots, n\}
$$

where $\eta \in(0,1]$ is the learning rate and is set close to zero to avoid oscillations in the learning process.

By the definition of a spiking neuron, the response of the output neurons is a function of the Postsynaptic Potential (PSP) induced by the input neurons. The PSP $\left(v^{i}\right)$ induced by the $i^{\text {th }}$ input neuron at time $t$ is represented as

$$
v^{i}(t)=\sum_{g} \epsilon\left(t-t_{i}^{(g)}\right)
$$

where $\epsilon\left(t-t_{i}^{(g)}\right)$ is the PSP induced by a spike on a synapse with unity weight due to a generic neuron model. For example, the value of $\epsilon(s)$ is given by the function $s / \tau * e^{1-s / \tau}$ for the spike response function [17], where $\tau$ is the time constant for the neuron. From Equation (5.3), the PSP of the $j^{\text {th }}$ output neuron $\left(v_{j}\right)$ at time $t$ can be mathematically expressed as

$$
v_{j}(t)=\sum_{i} w_{i j} v^{i}(t)
$$

When the PSP of the $j^{\text {th }}$ output neuron exceeds its threshold $\left(\theta_{j}\right)$, it generates a spike and its PSP is reset to zero.

To ensure that the $j^{\text {th }}$ output neuron spikes at $\bar{t}_{j}^{(g)}$ (Equation (5.2)), its weights should be updated such that the change in its PSP $\left(\Delta v_{j}^{(g)}\right)$ between the PSP at the actual time 
of the spike $\left(\hat{t}_{j}^{(g)}\right)$ and at the updated time of the spike $\left(\bar{t}_{j}^{(g)}\right)$ is given by

$$
\Delta v_{j}^{(g)}=\theta_{j}-v_{j}\left(\bar{t}_{j}^{(g)}\right)
$$

$\Delta v_{j}^{(g)}$ represents the required change in PSP of the $j^{\text {th }}$ output neuron to guarantee that it spikes at $\bar{t}_{j}^{(g)}$ for the current sample $(\mathbf{x})$. Suppose, $\Delta \mathbf{w}_{j}=\left[\Delta w_{1 j}, \cdots, \Delta w_{i j}, \cdots, \Delta w_{m j}\right]$ represents the change in weights of the $j^{\text {th }}$ output neuron to ensure that it spikes at $\bar{t}_{j}^{(g)}$. Then, the PSP of the $j^{\text {th }}$ output neuron at $\bar{t}_{j}^{(g)}$, due to the changed weights must be equal to $\Delta v_{j}^{(g)}$, which can be equivalently written as

$$
\sum_{i} \sum_{g} \Delta w_{i j} \epsilon\left(\bar{t}_{j}^{(g)}-t_{i}^{(g)}\right)=\Delta v_{j}^{(g)}
$$

It may be noted that $\Delta v_{j}^{(g)}$ will be positive when $\bar{t}_{j}^{(g)}<\hat{t}_{j}^{(g)}$ and it will be negative when $\bar{t}_{j}^{(g)}>\hat{t}_{j}^{(g)}$ assuming the slope of PSP for the $j^{t h}$ neuron with respect to time is positive at all times between $\hat{t}_{j}^{(g)}$ and $\bar{t}_{j}^{(g)}$. From Equation (5.6), the change in PSP $\left(\Delta v_{i j}^{(g)}\right)$ of the $j^{\text {th }}$ output neuron at $\bar{t}_{j}^{(g)}$ due to the $i^{\text {th }}$ input feature is given by

$$
\Delta v_{i j}^{(g)}=\sum_{g} \Delta w_{i j} \epsilon\left(\bar{t}_{j}^{(g)}-t_{i}^{(g)}\right)
$$

The proportion of $\Delta v_{j}^{(g)}$ contributed by the $i^{t h}$ input neuron $\left(\Delta v_{i j}^{(g)}\right)$ is calculated based on the weight sensitivity modulation factor for the synapse between the $i^{\text {th }}$ input neuron and the $j^{\text {th }}$ output neuron. The weight sensitivity modulation factor for a given synapse is determined by the meta-neuron such that the change in weight accurately captures the relationship between the input spike patterns and the desired output spike patterns. Next, the procedure used by the meta-neuron to determine the weight sensitivity modulation factor for a given synapse is described.

In the neuroscience literature, it has been shown that astrocytes selectively regulate the synaptic plasticity. Similarly, the meta-neuron based learning rule selectively updates the weights of the synapses connected to the postsynaptic neuron. For this purpose, the meta-neuron compares the existing knowledge stored in the network with respect to the knowledge in the current input spike pattern. The knowledge present in the current input 
spike pattern is represented by the normalized PSP $\left(u_{i}\right)$ induced by the $i^{\text {th }}$ input neuron at $\bar{t}_{j}^{(g)}$, given as

$$
u_{i}\left(\bar{t}_{j}^{(g)}\right)=\frac{v^{i}\left(\bar{t}_{j}^{(g)}\right)}{\sum_{i} v^{i}\left(\bar{t}_{j}^{(g)}\right)}
$$

The meta-neuron selects only those synapses for updating whose weights are lower than the normalized PSP for the current input spike pattern. This selective approach to modulating synaptic plasticity prevents a continuous increase in the weights of the potentiated synapses that is induced by STDP [9]. To compute the appropriate weight sensitivity modulation factor for a given synapse, the meta-neuron weights are initialized as

$$
z_{i}=\left\{\begin{array}{cc}
u_{i}\left(\bar{t}_{j}^{(g)}\right)-w_{i j} & \text { if } u_{i}\left(\bar{t}_{j}^{(g)}\right)>w_{i j} \\
0 & \text { otherwise }
\end{array} \quad i=1, \cdots, m\right.
$$

From Equation (5.9), one may notice that the meta-neuron weights are initialized to zero for a synapse when the normalized PSP of the corresponding input neuron for the current sample $(\mathbf{x})$ at $\left(\bar{t}_{j}^{(g)}\right)$ is lower than its existing weight. The input neurons whose weights are initialized to zero will have a weight sensitivity modulation factor of zero and, hence, their weights are not updated for the current input sample.

After the initialization of meta-neuron weights using the Equation (5.9), the contribution of the $i^{t h}$ input neuron to the meta-neuron PSP $\left(V^{i}\right)$ at $\bar{t}_{j}^{(g)}$ is given as

$$
V^{i}\left(\bar{t}_{j}^{(g)}\right)=\sum_{g} z_{i} \epsilon\left(\bar{t}_{j}^{(g)}-t_{i}^{(g)}\right)
$$

From Equation (5.10), the total PSP $(V)$ of the meta-neuron at $\bar{t}_{j}^{(g)}$ is given by

$$
V\left(\bar{t}_{j}^{(g)}\right)=\sum_{i} V^{i}\left(\bar{t}_{j}^{(g)}\right)
$$

From Equation (5.10) and (5.11), the meta-neuron estimates the weight sensitivity modulation factor $\left(M_{i j}\right)$ for the $i^{\text {th }}$ synapse of the $j^{\text {th }}$ output neuron as

$$
M_{i j}=\frac{\sum_{g} z_{i} \epsilon\left(\bar{t}_{j}^{(g)}-t_{i}^{(g)}\right)}{\sum_{i} \sum_{g} z_{i} \epsilon\left(\bar{t}_{j}^{(g)}-t_{i}^{(g)}\right)}
$$


which can be equivalently written as

$$
M_{i j}=\frac{V^{i}\left(\bar{t}_{j}^{(g)}\right)}{V\left(\bar{t}_{j}^{(g)}\right)}, \quad i \in\{1, \cdots, m\}
$$

It may be observed from Equation (5.12) that $M_{i j}$ will always lie in the interval $[0,1]$ and $\sum_{i} M_{i j}=1$. When it is equal to one for a given synapse of the $j^{\text {th }}$ neuron, then the weight sensitivity modulation factor for all the other synapses of the $j^{\text {th }}$ neuron will be equal to zero. This implies that the required change in PSP of the $j^{\text {th }}$ output neuron $\left(\Delta v_{j}^{(g)}\right)$ will be totally contributed by the synapse having a weight sensitivity modulation factor of unity. Thus, there will be no change in the weights of the other synapses having a weight sensitivity modulation factor of zero.

The weights of each synapse are updated based on the weight sensitivity modulation factor and the required change in the PSP of the $j^{\text {th }}$ neuron. The required change in PSP of the $j^{\text {th }}$ output neuron due to the $i^{\text {th }}$ input neuron $\left(\Delta v_{i j}^{(g)}\right)$ is determined using the weight sensitivity modulation factor as

$$
\Delta v_{i j}^{(g)}=M_{i j} \Delta v_{j}^{(g)}, \quad i \in\{1, \cdots, m\}
$$

The overall change in PSP of the $j^{\text {th }}$ output neuron should be equal to $\Delta v_{j}^{(g)}$ to ensure that it spikes at $\bar{t}_{j}^{(g)}$. Therefore, the weights of the $j^{t h}$ output neuron should be adjusted such that the actual change in PSP (given by Equation (5.7)) should be equal to the change in PSP determined based on the weight sensitivity modulation factor (given by Equation (5.14)) which is equivalently written as

$$
\sum_{g} \Delta w_{i j} \epsilon\left(\bar{t}_{j}^{(g)}-t_{i}^{(g)}\right)=M_{i j} \Delta v_{j}^{(g)}, \quad i \in\{1, \cdots, m\}
$$

The Equation (5.15) represents the condition that must be satisfied such that the $j^{\text {th }}$ neuron spikes precisely at $\bar{t}_{j}^{(g)}$. This determines the necessary changes in the weights of the synapses between the $i^{\text {th }}$ input feature and the $j^{\text {th }}$ output neurons as

$$
\Delta w_{i j}=M_{i j} \frac{\Delta v_{j}^{(g)}}{\sum_{g} \epsilon\left(\bar{t}_{j}^{(g)}-t_{i}^{(g)}\right)}, \quad i \in\{1, \cdots, m\}
$$


The meta-neuron based learning rule, given by Equation (5.16) is used to update the synaptic weights for each spike generated by the $j^{\text {th }}$ output neuron to ensure that it learns to spike closer to the desired spike times. The meta-neuron based learning rule in Equation (5.16) is similar to the learning rule for self-organizing maps [91]. The ability of the learning rule to produce precise shifts in the spike times of the output neurons makes it an effective update mechanism in an online learning framework. To demonstrate this capability of the learning rule, an online meta-neuron based learning algorithm for pattern classification problems is presented in the next section.

\subsection{Online Meta-neuron Based Learning Algorithm for Pattern Classification Problems}

In this section, an online meta-neuron based learning algorithm for a two layered spiking neural network for pattern classification problems is developed. The aim of the learning algorithm is to closely approximate the relationship between the input spike patterns and the corresponding class labels in a single presentation of the training samples. The predicted class for a given sample is determined based on the class association of that output neuron which spikes first (output neuron having a minimum latency). As a result, there is a need to encode the true class label in the form of a spike pattern. Since latency of the neuron is used to determine the class label, the learning algorithm trains the network to generate the first spike for a given sample at the target firing time. The target firing time for a neuron belonging to the true class is set to a fixed time instant in the simulation interval, denoted by $T_{I D}$. For other class neurons, the target firing time is set to $T+\delta$, which implies that other class neurons should not generate a spike within the simulation interval.

The Online Meta-neuron based Learning Algorithm (OMLA) utilizes the meta-neuron based learning rule to learn and evolve the network in an online framework. It chooses one of the three different heuristic strategies, namely, add a neuron, delete the sample or update the network parameters for learning a given input sample. The right learning strategy is chosen based on the spike pattern generated by the output neurons. This approach has been previously used in the self-regulating evolving spiking neural [82] 
classifier working in a batch learning environment. Here, OMLA uses similar heuristics to learn each sample in one-shot using the meta-neuron based learning rule.

The learning algorithm stores those training samples in meta-neuron memory that are used to add a neuron by the learning algorithm. The stored samples act as pseudoinputs that represent the knowledge stored in the network. While adding a neuron for a subsequent sample, these pseudo-inputs are used by the learning algorithm for a better approximation of the past knowledge in the network.

Without loss of generality, it is assumed that the network has $K$ output neurons, which were added while learning the spike patterns $\mathbf{x}_{1}, \cdots, \mathbf{x}_{h}, \cdots, \mathbf{x}_{K}$. At this juncture, the meta-neuron's memory will contain these samples that have been used to add the $K$ neurons. Next, the current sample $\mathbf{x}$ from class $c$ is presented to the network for learning. The following discussion represents the spike time of the output neuron having minimum latency from class $c$ as $t_{C C}\left(C C\right.$ implies correct class). Similarly, $t_{M C}$ represents the spike time of the output neuron with minimum latency from any other class. Suppose $c_{j}$ is the class associated with the $j^{\text {th }}$ output neuron, then the neurons $C C$ and $M C$ are given as

$$
\begin{gathered}
C C=\underset{j, c_{j}=c}{\operatorname{argmin}} t_{j} \\
M C=\underset{j, c_{j} \neq c}{\operatorname{argmin}} t_{j}
\end{gathered}
$$

It may be noted that the time of first spike generated by the output neuron is represented as $t_{j}$ (instead of $t_{j}^{(1)}$ ) as the learning algorithm uses only the first spike for learning and prediction. Next, the different learning strategies employed by the learning algorithm are given in detail.

- Neuron addition strategy: In this strategy, the learning algorithm chooses to add a neuron to the output layer when the current sample contains a significant amount of new knowledge. A sample is considered to contain a significant amount of new knowledge when the time interval between $T_{I D}$ and $t_{C C}$ is very high $\left(T_{I D}<<\right.$ $\left.t_{C C}\right)$. A fixed time instant, $T_{n} \in\left[T_{I D}, T\right]$, is used as a threshold for $t_{C C}$ to identify 
the samples for neuron addition. Thus, the criteria for neuron addition is given as,

$$
\text { If } t_{C C}>T_{n}
$$

Then a neuron is added to the network

where $T_{n}$ is given by

$$
T_{n}=\alpha_{n} T+\left(1-\alpha_{n}\right) T_{I D}
$$

Here, $\alpha_{n}$ is termed as novelty threshold and is always set to a value in the interval $[0,1]$. If its value is set closer to zero, the learning algorithm adds a neuron to the network for each training sample resulting in overfitting. If it is set closer to one, the learning algorithm adds too few neurons to the network, resulting in an imprecise model of the data. It should be set to a value closer to one, to ensure proper generalization of the trained network on unseen samples. A suitable range for initializing $\alpha_{n}$ is $[0.7,1]$.

The weights of the synapse between the $i^{\text {th }}$ input neuron and the newly added neuron are initialized according to the normalized PSP (computed from Equation (5.8)) induced by the $i^{\text {th }}$ input neuron at $T_{I D}$. Hence, the weights $\left(\mathbf{w}_{(K+1)}=\right.$ $\left.\left[w_{1(K+1)}, \cdots, w_{i(K+1)}, \cdots, w_{m(K+1)}\right]\right)$ of a newly added neuron are given as

$$
w_{i(K+1)}=u_{i}\left(T_{I D}\right)
$$

The threshold $\left(\theta_{(K+1)}\right)$ for the neuron is initialized as

$$
\theta_{(K+1)}=\sum_{i} \sum_{g} w_{i(K+1)} \epsilon\left(T_{I D}-t_{i}^{(g)}\right)
$$

The initialization of neuron weights and threshold in this manner does not take into account the past knowledge stored in the network. In order to capture the past knowledge while adding a neuron, the samples used to evolve the network (samples stored in meta-neuron memory) are used as pseudo-inputs representing the past knowledge in the network. If the newly added neuron fires closer to $T_{I D}$ for the pseudo-inputs from other classes, then its weights are updated such that it fires late 
for other class samples.

Suppose $t_{K+1}^{[h]}$ is the time of the first spike generated by the $(K+1)^{t h}$ neuron for the $h^{\text {th }}$ sample in the meta-neuron memory. To ensure that the newly added neuron does not fire sooner for samples from class $c_{h}, t_{K+1}^{[h]}$ should be much higher than $t_{h}^{[h]}$. To identify the pseudo-inputs used for updating the weights of the $(K+1)^{t h}$ neuron, a fixed time duration, $T_{m}$ is used as a threshold for the time difference between $t_{K+1}^{[h]}$ and $t_{h}^{h}$. Hence, the criterion for updating the weights of the newly added neuron due to the $h^{\text {th }}$ sample in the meta-neuron memory is given by

$$
\text { If } t_{K+1}^{[h]}-t_{h}^{[h]}<T_{m}, \quad h \in\{1, \cdots, K\}, c_{h} \neq c_{K+1}
$$

Then adjust the weights of the $(K+1)^{t h}$ neuron

where $T_{m}$ is given by

$$
T_{m}=\alpha_{m}\left(T-T_{I D}\right)
$$

Here, $\alpha_{m}$ is termed as the margin threshold. The intent of the margin threshold $\left(\alpha_{m}\right)$ is similar to the problem of margin maximization for SVM [84]. It is always initialized to a value in the interval $[0,1]$. If it is set closer to zero, the network will not generalize well due to smaller interclass margin. If it is set closer to one, then the network may loose knowledge gained from past samples. The experimental analyses showed that the performance of the learning algorithm is acceptable when $\alpha_{m}$ is set in the interval $[0,0.3]$. The value of $\alpha_{m}$ is set to 0.3 for all simulations described in this work.

The weights of the newly added neuron are updated using the meta-neuron based learning rule and its new spike time for the $h^{\text {th }}$ sample after the weight update is given as

$$
\bar{t}_{K+1}^{[h]}=t_{h}^{[h]}+T_{m}
$$

- Delete sample strategy: The current sample is deleted when the $t_{C C}$ is very close to $T_{I D}$ and $t_{M C}$ is much larger than $t_{C C}$. When $t_{C C}$ is close to $T_{I D}$, it indicates that the current sample does not contain a significant amount of new knowledge. Further, a considerable time difference between $t_{C C}$ and $t_{M C}$ implies that the current 
sample is well separated from the other class samples. To determine those samples that can be deleted from the learning process, a fixed time instant, $T_{d} \in\left[T_{I D}, T\right]$, is used as a threshold for $t_{C C}$. This strategy prevents the network from learning similar samples repeatedly, thereby preventing overtraining and also reduces the computational requirements of the learning algorithm. The criteria for this strategy is given as:

$$
\text { If } t_{C C} \leq T_{d} \quad \& \quad\left(t_{M C}-t_{C C}\right) \geq T_{m}
$$

Then the current input sample is deleted

where $T_{d}$ is given as

$$
T_{d}=\alpha_{d} T+\left(1-\alpha_{d}\right) T_{I D}
$$

Here, $\alpha_{d}$ is termed as the delete threshold and is used to determine the samples that can be discarded from the learning process. It is always set to a value in the interval $[0,1]$. If it is set closer to zero, it will result in all samples being learnt by the learning algorithm which will lead to a lower generalization performance. If it is set closer to one, it will result in the deletion of too many samples resulting in the generation of an imprecise model of data. Based on the simulation studies, it was observed that, a suitable range for $\alpha_{d}$ is $[0,0.25]$. The performance of the learning algorithm is satisfactory when $\alpha_{d}$ is set in this interval. Its values is fixed at 0.25 for all the simulations presented in this work.

- Parameter update strategy: The learning algorithm chooses to update the synaptic weights of existing neurons when the criterion for none of the above strategies of neuron addition or delete sample is satisfied. The aim of this strategy is to update the synaptic weights such that $t_{C C}$ is close to $T_{I D}$ and there exists a high time difference between $t_{C C}$ and $t_{M C}$, for all the samples. The weights of the neuron $C C$ are updated to ensure that the correct class neuron fires close to $T_{I D}$. Further, the learning algorithm also updates the weights of the neuron $M C$ to ensure a higher time difference exists between $t_{C C}$ and $t_{M C}$.

The weights of the neuron $C C$ are updated using the meta-neuron based learning 
rule and the spike time $\left(\bar{t}_{C C}\right)$ of the neuron $C C$ after the weight update is given as

$$
\bar{t}_{C C}=t_{C C}-\alpha_{s} t_{C C}
$$

where $\alpha_{s}$ is termed as the learning rate and is always initialized to a value in the interval $[0,1]$. A high value of the learning rate causes oscillations in the learning process. Hence, a suitable range for initializing $\alpha_{s}$ is $[0,0.1]$.

To improve the margin between $t_{C C}$ and $t_{M C}$, the weights of the neuron $M C$ are updated using the meta-neuron based learning rule when $\left(t_{M C}-\bar{t}_{C C}\right)<T_{m}$. The spike time for the neuron $M C$ after the weight update is given by

$$
\bar{t}_{M C}=\bar{t}_{C C}+T_{m}
$$

The utilization of global as well as local information by the meta-neuron enables the learning algorithm to estimate the changes in weights such that the relationship between the input spike patterns and the corresponding class labels is closely approximated in a single presentation. Using a meta-neuron with memory for storing samples used to evolve the network allows the learning algorithm to approximate the past knowledge properly while adding a neuron.

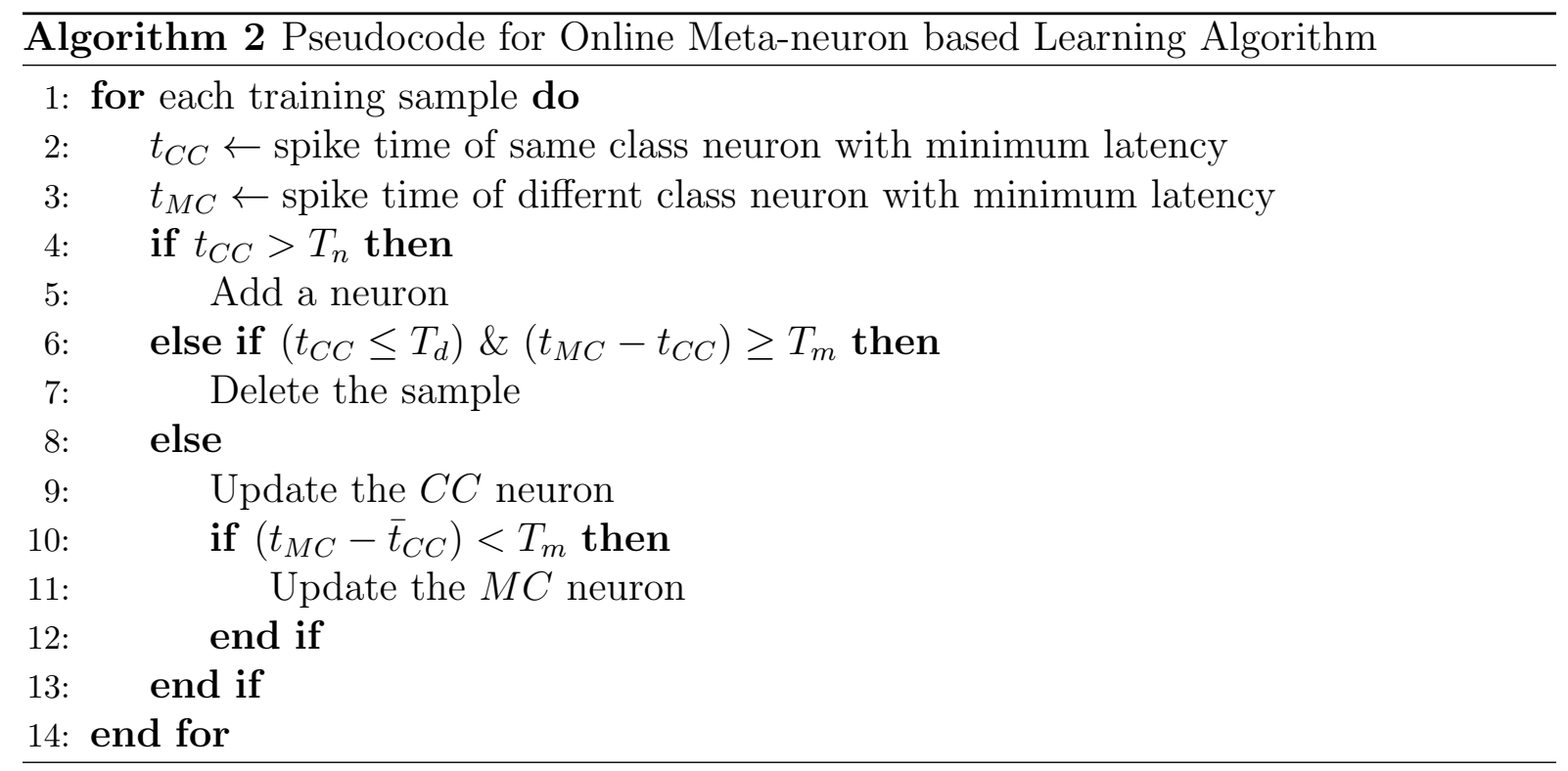


A summary of the online meta-neuron based learning algorithm in a pseudocode format is given in the Algorithm 2. Next, the working of the OMLA is illustrated using the Ionosphere problem from the UCI machine learning repository. Based on this study, guidelines are also provided for appropriately setting the algorithm parameters, addition threshold and learning rate.

\subsection{Working of OMLA Using the Ionosphere Prob- lem}

In this section, the working of the learning algorithm is described using the Ionosphere problem from the UCI machine learning repository. The Ionosphere problem is also used to describe the effect of the different algorithm parameters on the performance of the learning algorithm, based on which suitable guidelines are suggested for setting the parameters to appropriate values.

The Ionosphere problem contains radar information collected from 16 high frequency receivers. The data set has in total 34 attributes and the problem is to determine whether the received signal conveys any information about the structure of the Ionosphere. It has a total of 351 samples, out of which 175 are used for training and the rest for testing.

The learning algorithm has four main parameters, namely, a novelty threshold $\left(\alpha_{n}\right)$, a margin threshold $\left(\alpha_{m}\right)$, a delete threshold $\left(\alpha_{d}\right)$ and the learning rate $\left(\alpha_{s}\right)$. As described earlier, the two parameters, viz. the margin threshold and the delete threshold values are fixed at 0.3 and 0.25 respectively. For initializing the novelty threshold and learning rate, the suitable ranges have been indicated earlier as $[0.7,1]$ and $[0,0.1]$ respectively. For example, when the novelty threshold and the learning rate are initialized to 0.73 and 0.09 respectively, the average training accuracy is $93.2 \%$ and the average testing accuracy is $93 \%$ for the Ionosphere problem. Out of the 175 samples, the learning algorithm used only 136 samples for learning and added 25 neurons to the network. Figure 5.2 shows the neuron growth history for the Ionosphere problem. The learning algorithm adds the last neuron for the $92^{\text {nd }}$ training sample and chooses only to update the network parameters or delete the sample from the learning process for the subsequent training samples. Next, the effects of the 'delete sample strategy' and the meta-neuron memory on the learning 


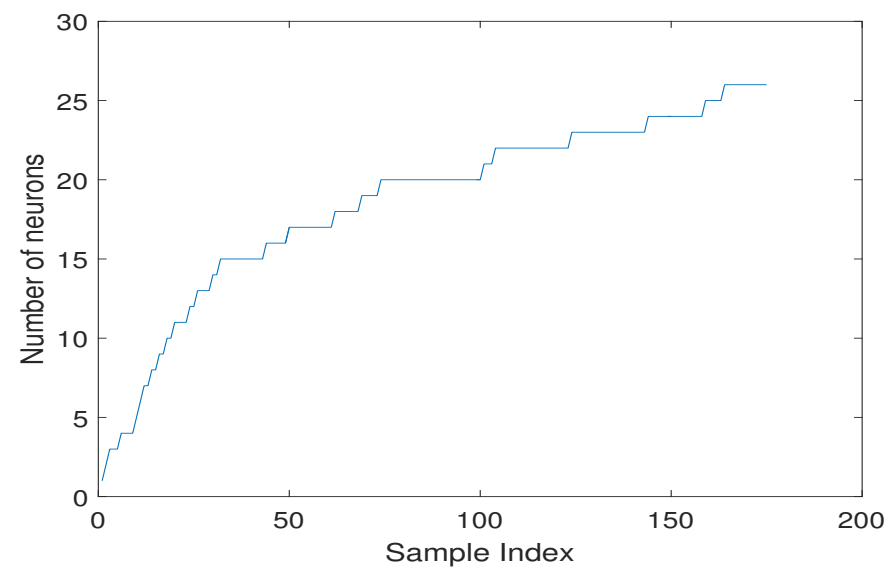

Figure 5.2: Neuron growth history of the learning algorithm for the Ionosphere problem

algorithm are described. Also, the impact of the algorithm parameters, viz. the novelty threshold and the learning rate on the performance of the learning algorithm is illustrated.

Effects of the delete sample strategy: Similar to the observed behavior in [92], it was observed that the learning algorithm achieves higher generalization accuracy when similar samples are deleted. For the Ionosphere problem, training and testing accuracy of $93.2 \%$ and $93 \%$ are obtained when the learning algorithm is trained with the 'delete sample strategy'. The learning algorithm deleted 39 samples from the training set of 175 samples (22\% is deleted). When the learning algorithm was trained without the 'delete sample strategy' all the samples were used in training. In this case a training and testing accuracy of $93.8 \%$ and $84.6 \%$, respectively are obtained. This clearly shows that the 'delete sample strategy' helps in improving the generalization performance of the learning algorithm.

Effect of meta-neuron memory: A similar study was conducted to analyze the impact of meta-neuron memory on the performance of the learning algorithm. When the learning algorithm is trained without the meta-neuron memory, the average training and testing performance are $85.71 \%$ and $79.55 \%$ respectively, while with the memory they are $93.2 \%$ and $93.0 \%$ respectively. This clearly highlights that, when the learning algorithm is trained without meta-neuron memory its performance drops considerably for the Ionosphere problem. In the absence of meta-neuron memory, the learning algorithm has no information about past knowledge stored in the network. As a result, newly 
added neurons do not approximate the past knowledge effectively. This results in lower performance in an online framework. The results clearly show that meta-neuron memory plays a vital role in improving the performance of the learning algorithm.

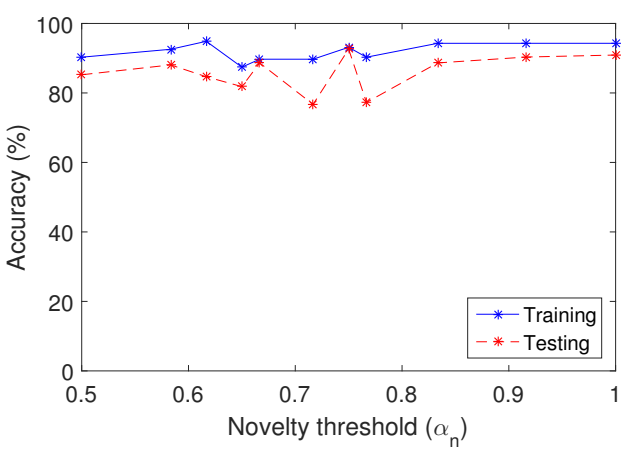

(a)

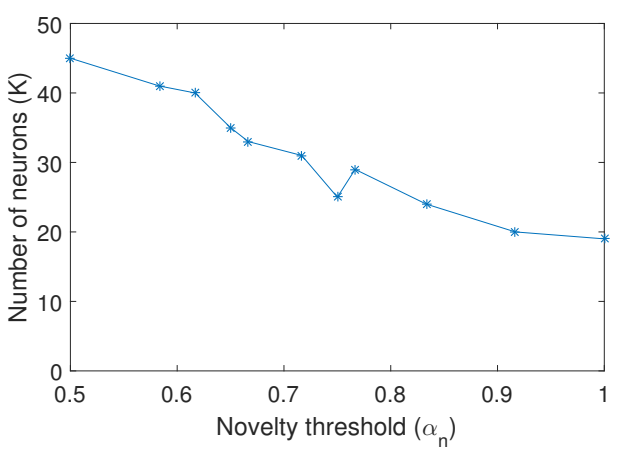

(b)

Figure 5.3: Effect of novlety threshold $\left(\alpha_{n}\right)$ on the (a) training and testing accuracy, (b) number of neurons required to approximate the decision function

Effect of novelty threshold: To illustrate the effect of the novelty threshold $\left(\alpha_{n}\right)$ on the learning algorithm, it was evaluated for values of $\alpha_{n}$ in the range [0.5,1]. Figure 5.3a shows both training and testing performance against $\alpha_{n}$. It can be seen from the figure that there is a small change in the training performance whereas there is a change of over $15 \%$ in testing performance as $\alpha_{n}$ is varied over the interval [0.5,1]. Figure 5.3b shows the impact of $\alpha_{n}$ on the number of neurons added by the learning algorithm to the network. It is seen that a lower value of $\alpha_{n}$ results in more neurons being added to the network and vice-versa, thereby, showing that $\alpha_{n}$ significantly impacts the generalization performance and the number of neurons added by the learning algorithm. Hence, it has to be chosen carefully. A suitable range for setting $\alpha_{n}$ is $[0.7,1]$ to achieve good performance using a compact network.

Effect of learning rate: In this experiment, the choice of learning rate $\left(\alpha_{s}\right)$ in the interval $[0,0.2]$ is studied. Figure $5.4 \mathrm{a}$ shows the impact of learning rate on both, the training and testing performance. It is seen that there is small variation in the training as well as testing performance when the learning rate is in the range $[0,0.1]$. When $\alpha_{s}$ is increased beyond 0.1 both the training as well as testing performance start deteriorating. This is because for a high value of $\alpha_{s}$ the network looses knowledge gained from previous samples. Figure 5.4b shows the variation in the number of neurons added by the learning 


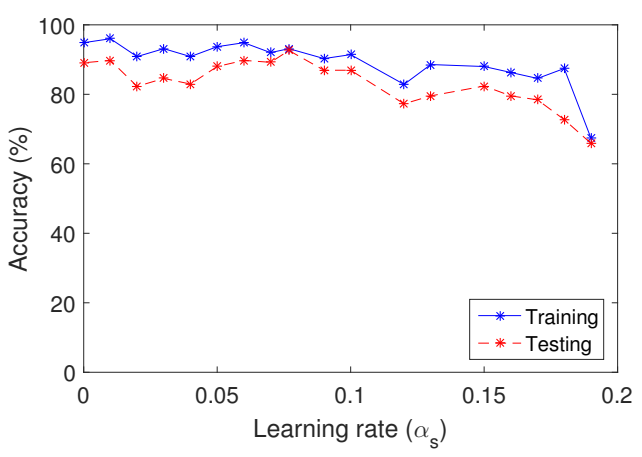

(a)

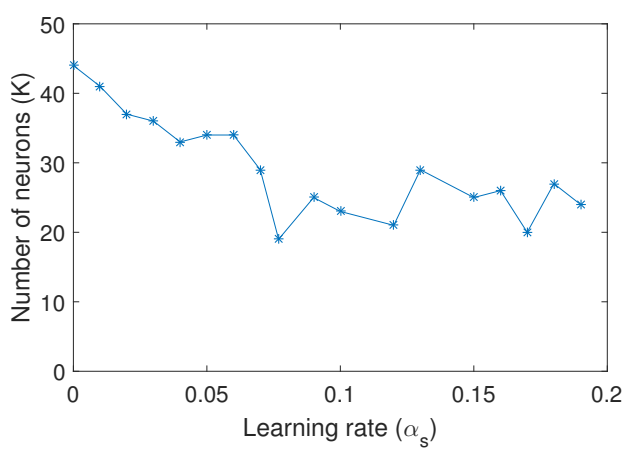

(b)

Figure 5.4: Effect of learning rate $\left(\alpha_{s}\right)$ on the (a) training and testing accuracy, (b) number of neurons required to approximate the decision function

algorithm as $\alpha_{s}$ is varied in the range $[0,0.2]$. When $\alpha_{s}$ is set to zero, it plays no role in the learning process. In such a scenario, more neurons are required by the network to ensure that the knowledge present in the samples is properly learnt by the network. This is evident from the plot shown in Figure 5.4b. To summarize, $\alpha_{s}$ affects both, the generalization performance as well as the architecture of the trained neural network. Based on these observations, it is recommended that $\alpha_{s}$ be set in the range $[0,0.1]$ for achieving good network performance.

Next, the performance of the learning algorithm is evaluated on benchmark problems from the UCI machine learning repository and the results of evaluation are compared with that of other spiking neural classifiers.

\subsection{Performance Evaluation of the Online Meta-neuron Based Learning Algorithm}

In this section, the performance of OMLA is studied using ten benchmark data sets from the UCI machine learning repository [21]. Table 5.1 highlights the details of the data sets used for comparison. The table provides information about the number of features, number of classes and number of training/testing samples for the data sets used in comparison. The results of performance evaluation have been compared with other existing online and batch learning algorithms for spiking neural networks. For 
Table 5.1: Description of the data sets used for evaluation

\begin{tabular}{|c|c|c|c|c|}
\hline Data set & \# Features & \# Classes & \multicolumn{2}{|c|}{ \# Samples } \\
\cline { 3 - 5 } & & & Training & Testing \\
\hline \hline Breast cancer & 9 & 2 & 350 & 333 \\
Echocardiogram & 10 & 2 & 66 & 65 \\
Mammogram & 9 & 2 & 80 & 11 \\
Liver & 6 & 2 & 170 & 175 \\
PIMA & 9 & 2 & 384 & 384 \\
Ionosphere & 34 & 2 & 175 & 176 \\
Hepatitis & 19 & 2 & 78 & 77 \\
\hline Iris & 4 & 3 & 75 & 75 \\
Wine & 13 & 3 & 60 & 118 \\
Acoustic emission & 5 & 4 & 62 & 137 \\
\hline
\end{tabular}

online learning algorithms, a comparison was made with Online Spiking Neural Network (OSNN) [81] and the SRESN classifier [82] in an online learning mode, referred as the online version of the SRESN classifier. In online learning mode, the performance of the SRESN classifier is evaluated by training the SRESN classifier for a single epoch using a high learning rate of 0.1 . The comparison with online learning algorithms is done for five problems i.e. Iris flower classification, Wisconsin breast cancer, Liver, PIMA and Ionosphere as the results for Online SNN are available only for these problems. With regards to batch learning algorithm, a comparison was done with three batch learning algorithms for SNNs, viz. SpikeProp [17], Synaptic Weight Association training (SWAT) [10] and the Two stage Margin Maximization Spiking Neural Network (TMM-SNN).

For all the simulation studies reported in this section, the spiking neurons in the output layer are modeled using the spike response function [17] and the time constant for the neuron is fixed at $3 \mathrm{~ms}$. The real valued data from the benchmark problems is encoded into spike patterns using the population coding scheme [17]. As described in [82], the overlap constant for the population coding has been fixed at 0.7 and six receptive fields have been used for converting the real valued data into spike patterns. Using the population coding, each receptive field generates a spike in the interval $[0,3] \mathrm{ms}$. Hence, the simulation interval has been set slightly higher than the range of input spikes, at 3.2 $m s$, to ensure that all spikes generated by the output layer neurons are recorded.

The performance of all the algorithms was evaluated based on overall training and 
testing accuracy, which is equal to the percentage of total number of samples that are correctly classified by the network. The average performance over ten random trials is used for the purpose of comparison. All the experiments have been carried out in Windows 7 in MATLAB 2014b using a CPU with 12 logical cores, 16 GB memory with a speed of $3.2 \mathrm{GHz}$.

For every data set, ten random sets are generated using the same number of training and testing samples (as suggested in [93]). The training and testing accuracy is computed for all the sets and the mean along with the standard deviation for the experiments is reported. The results for all the other algorithms have been generated by us except for the OSNN whose results have been reproduced from [81]. As in the SpikeProp paper [17], the results for SpikeProp are generated using 16 delays per receptive field, a learning rate of 0.0075 , coding interval of 4 milliseconds and a time constant of 7 milliseconds. The number of neurons in the hidden layer for SpikeProp is determined using the constructivedestructive procedure [85]. For SWAT, the important parameters are $c_{o}$ and the maximum height of the plasticity window $\left(A_{p}\right)$. The other parameters pertaining to the neuron model and frequency filtering are set as mentioned in the [10]. It has been highlighted in [10] that, a suitable value of $c_{o}$ depends on the number of epochs required for convergence. We observed during the experiments that, SWAT converged within 500 epochs for all the data sets evaluated in this thesis. Hence, $c_{o}$ was set to 4000 as in [10]. The impact of

Table 5.2: Parameter values for novelty threshold $\left(\alpha_{n}\right)$ and learning rate $\left(\alpha_{s}\right)$ for benchmark data sets used for comparison

\begin{tabular}{|c|c|c|}
\hline Data set & Novelty & Update \\
\cline { 2 - 3 } & Threshold $\left(\alpha_{n}\right)$ & Factor $\left(\alpha_{s}\right)$ \\
\hline Breast cancer (BC) & 0.96 & 0.06 \\
Echocardiogram (ECHO) & 0.77 & 0.06 \\
Mammogram (MAM) & 0.77 & 0.06 \\
Liver & 0.98 & 0.05 \\
PIMA & 0.80 & 0.04 \\
Ionosphere (ION) & 0.73 & 0.09 \\
Hepatitis (HEP) & 0.77 & 0.04 \\
\hline \hline Iris & 0.70 & 0.06 \\
Wine & 0.73 & 0.05 \\
Acoustic emission (AE) & 0.79 & 0.04 \\
\hline
\end{tabular}


$A_{p}$ is similar to the effect of learning rate on other learning algorithms and has been set in the interval $[0.1,0.5]$ in $[10]$. In this work, $A_{p}$ is fixed at 0.1 to avoid oscillations in the learning process. For OMLA, the parameter values for delete threshold and margin threshold in OMLA are fixed at 0.25 and 0.3 , respectively. The parameter values for the novelty threshold $\left(\alpha_{n}\right)$ and the learning rate $\left(\alpha_{s}\right)$ for all the data sets, have been selected using 10-fold cross validation and are given in the Table 5.2.

Table 5.3 shows the architecture and the results of the performance evaluation for the online learning algorithms. The architectures of OMLA is shown in the format $(m: K)$ as it employs a two layered networks. The other algorithms employ a three layered architectures, hence, the number of hidden neurons are also shown. For evolving learning algorithms (OSNN, TMM-SNN and OMLA) the architecture shows the range of neurons added by the learning algorithm for the ten random trials. It may be noted that the number of input neurons in the architecture of the different learning algorithms is equal to the product of the number of features and the number of receptive fields used for population coding.

Table 5.3: Performance comparison of OMLA with OSNN and the Online SRESN

\begin{tabular}{|c|c|c|c|c|}
\hline $\begin{array}{c}\text { Data } \\
\text { set }\end{array}$ & $\begin{array}{c}\text { Benchmark } \\
\text { criterion }\end{array}$ & OMLA & OSNN & $\begin{array}{c}\text { Online } \\
\text { SRESN }\end{array}$ \\
\hline \hline \multirow{3}{*}{ Iris } & Architecture & $24:(5-7)$ & $48:(7-21): 3$ & $24:(6-11)$ \\
& Training & $\mathbf{9 7 . 9 ( 0 . 7 )}$ & $87.2(4.1)$ & $92.7(4.2)$ \\
& Testing & $\mathbf{9 7 . 9 ( 0 . 7 )}$ & $86.1(6.7)$ & $93.0(5.7)$ \\
\hline \multirow{3}{*}{ BC } & Architecture & $54: 2$ & $54:(10-16): 2$ & $54:(5-8)$ \\
& Training & $\mathbf{9 7 . 4 ( 0 . 4 )}$ & $91.1(2.0)$ & $93.9(1.8)$ \\
& Testing & $\mathbf{9 7 . 8 ( 0 . 4 )}$ & $90.4(1.8)$ & $94.0(2.6)$ \\
\hline \multirow{4}{*}{ Liver } & Architecture & $36:(12-15)$ & $36:(4-7): 2$ & $36:(5-8)$ \\
& Training & $\mathbf{6 9 . 9}(\mathbf{2 . 3})$ & $58.7(2.2)$ & $59.8(1.2)$ \\
& Testing & $\mathbf{6 7 . 7}(\mathbf{1 . 8})$ & $56.7(1.8)$ & $57.4(1.1)$ \\
\hline \multirow{4}{*}{ PIMA } & Architecture & $54: 20$ & $54:(8-18): 2$ & $54:(6-12)$ \\
& Training & $\mathbf{7 8 . 6 ( 1 . 7 )}$ & $68.2(2.0)$ & $67.0(0.8)$ \\
& Testing & $\mathbf{7 7 . 9}(\mathbf{1 . 0})$ & $63.5(3.0)$ & $66.1(1.4)$ \\
\hline \multirow{3}{*}{ ION } & Architecture & $204:(19-25)$ & $204:(4-11): 2$ & $204:(6-13)$ \\
& Training & $\mathbf{9 4 . 0}(\mathbf{1 . 7})$ & $76.7(2.4)$ & $85.1(1.9)$ \\
& Testing & $\mathbf{9 3 . 5 ( 0 . 5 )}$ & $76.6(4.8)$ & $79.3(3.0)$ \\
\hline
\end{tabular}

It can be observed from the Table 5.3 that, OMLA performs significantly better than the other online learning algorithms. For further discussion, the performance of OMLA is compared only with the performance of Online SRESN as Online SRESN performs better than OSNN. For simple problems like Iris flower classification and Wisconsin breast 
cancer the performance of OMLA is $3 \%$ to $4 \%$ better than the performance of Online SRESN. For low dimensional problems with lower separability like Liver and PIMA, OMLA performs 10-11\% better than Online SRESN. For a high dimensional problem like Ionosphere, OMLA performs $14 \%$ better than Online SRESN. Next, a statistical analysis of the performance comparison is presented.

Statistical analysis of performance comparison: A one-way ANOVA test was conducted to analyze the results of the performance comparison between OMLA and other online learning algorithms. The statistical test was conducted with the null hypothesis that the performance of the three algorithms do not differ significantly. If the $p$-value for the computed $F$-statistic is lower than 0.05 (95\% confidence interval) then the null hypothesis is rejected. In this study, the mean testing accuracy of the three algorithms for the five data sets represents three different groups and ANOVA monitors the variations between the groups. An $F$-statistic of 31.87 was obtained for group-wise variation which corresponds to a $p$-value of 0.0002 . Hence, one can reject the null hypothesis with a 95\% confidence interval. Thereafter, a pairwise comparison was performed between the three classifiers using the Bonferroni method. The observed $p$-values for the pairwise comparison of OMLA and Online SRESN was 0.0015 and for the pairwise comparison of OMLA and OSNN was 0.0002. Since, both the $p$-values are lower than 0.05 (95\% confidence interval), it can be concluded that OMLA performs better than the other algorithms used for comparison with a $95 \%$ confidence interval. Next, the performance results of OMLA are compared with other existing batch learning algorithms for spiking neural networks.

Table 5.4 shows the results of comparison with other batch learning algorithms. Among other batch learning algorithms, TMM-SNN performs better than the other algorithms. Hence, further discussion is restricted to a comparison between the performance of OMLA and TMM-SNN. It can be observed from the table, that for all problems except LIVER the performance of OMLA is similar to the performance of TMM-SNN. In case of Liver problem, TMM-SNN performs better than OMLA.

These observations clearly highlight that the utilization of global information present in the network as well as the local information present in the input spike patterns help the meta-neuron based learning rule in effectively updating the synaptic weights in one-shot. 
Table 5.4: Performance comparison of OMLA with SpikeProp, SWAT and TMM-SNN

\begin{tabular}{|c|c|c|c|c|c|}
\hline $\begin{array}{c}\text { Data } \\
\text { set }\end{array}$ & $\begin{array}{l}\text { Learning } \\
\text { Algorithm }\end{array}$ & Architecture & $\begin{array}{c}\text { Training } \\
\text { Accuracy }(\%)\end{array}$ & $\begin{array}{c}\text { Testing } \\
\text { Accuracy (\%) }\end{array}$ & $\begin{array}{c}\# \\
\text { Epochs }\end{array}$ \\
\hline \multirow{4}{*}{$\mathrm{BC}$} & SpikeProp & $55-15-2$ & $97.3(0.6)$ & $97.2(0.6)$ & 1000 \\
\hline & SWAT & $54-702-2$ & $96.5(0.5)$ & $95.8(1.0)$ & 500 \\
\hline & TMM-SNN & $54-(2-8)-2$ & $97.4(0.3)$ & $97.2(0.5)$ & 70 \\
\hline & OMLA & $54-2$ & $97.4(0.4)$ & $97.8(0.4)$ & 1 \\
\hline \multirow{4}{*}{$\mathrm{ECHO}$} & SpikeProp & 61-10-2 & $86.6(2.5)$ & $84.5(3.0)$ & 1000 \\
\hline & SWAT & $60-780-2$ & $90.6(1.8)$ & $81.8(2.8)$ & 500 \\
\hline & TMM-SNN & $60-(2-3)-2$ & $86.5(2.1)$ & $85.4(1.7)$ & 177 \\
\hline & OMLA & $60-(6-10)$ & $89.6(1.5)$ & $86.3(0.9)$ & 1 \\
\hline \multirow{4}{*}{ MAM } & SpikeProp & $55-10-2$ & $82.8(4.7)$ & $81.8(6.1)$ & 1000 \\
\hline & SWAT & $54-702-2$ & $82.6(2.1)$ & $78.2(12.3)$ & 500 \\
\hline & TMM-SNN & $54-(5-7)-2$ & $87.2(4.4)$ & $84.9(8.6)$ & 176 \\
\hline & OMLA & 54-(14-20) & $88.6(2.0)$ & $85.5(4.1)$ & 1 \\
\hline \multirow{4}{*}{ Liver } & SpikeProp & $37-15-2$ & $71.5(5.2)$ & $65.1(4.7)$ & 3000 \\
\hline & SWAT & $36-468-2$ & $74.8(2.1)$ & $60.9(3.2)$ & 500 \\
\hline & TMM-SNN & $36-(5-8)-2$ & $74.2(3.5)$ & $70.4(2.0)$ & 442 \\
\hline & OMLA & $36-(12-15)$ & $69.9(2.3)$ & $67.7(1.8)$ & 1 \\
\hline \multirow{4}{*}{ PIMA } & SpikeProp & $55-20-2$ & $78.6(2.5)$ & $76.2(1.8)$ & 3000 \\
\hline & SWAT & $54-702-2$ & $77.0(2.1)$ & $72.1(1.8)$ & 500 \\
\hline & TMM-SNN & $54-(5-14)-2$ & $79.7(2.3)$ & $78.1(1.7)$ & 160 \\
\hline & OMLA & $54-20$ & $78.6(1.7)$ & $77.9(1.0)$ & 1 \\
\hline \multirow{4}{*}{ ION } & SpikeProp & $205-25-2$ & $89.0(7.9)$ & $86.5(7.2)$ & 3000 \\
\hline & SWAT & $204-2652-2$ & $86.5(6.7)$ & $90.0(2.3)$ & 500 \\
\hline & TMM-SNN & $204-(23-34)-2$ & $98.7(0.4)$ & $92.4(1.8)$ & 246 \\
\hline & OMLA & $204-(19-25)$ & $94.0(1.7)$ & $93.5(0.5)$ & 1 \\
\hline \multirow{4}{*}{ HEP } & SpikeProp & $115-15-2$ & $87.8(5.0)$ & $83.5(2.5)$ & 1000 \\
\hline & SWAT & $114-1482-2$ & $86.0(2.1)$ & $83.1(2.2)$ & 500 \\
\hline & TMM-SNN & 114-(3-9)-2 & $91.2(2.5)$ & $86.6(2.2)$ & 192 \\
\hline & OMLA & $114-12$ & $89.8(2.9)$ & $87.4(2.4)$ & 1 \\
\hline \multirow{4}{*}{ Iris } & SpikeProp & $25-10-3$ & $97.2(1.9)$ & $96.7(1.6)$ & 1000 \\
\hline & SWAT & $24-312-3$ & $96.7(1.4)$ & $92.4(1.7)$ & 500 \\
\hline & TMM-SNN & $24-(4-7)-3$ & $97.5(0.8)$ & $97.2(1.0)$ & 94 \\
\hline & OMLA & $24-(5-7)$ & $97.9(0.7)$ & $97.9(0.7)$ & 1 \\
\hline \multirow{4}{*}{ Wine } & SpikeProp & $79-10-2$ & $99.2(1.2)$ & $96.8(1.6)$ & 1000 \\
\hline & SWAT & 78-1014-3 & $98.6(1.1)$ & $92.3(2.4)$ & 500 \\
\hline & TMM-SNN & $78-3-3$ & $100(0)$ & $97.5(0.8)$ & 80 \\
\hline & OMLA & $78-(3-6)$ & $98.5(1.0)$ & $97.9(0.7)$ & 1 \\
\hline \multirow{4}{*}{$\mathrm{AE}$} & SpikeProp & $31-10-4$ & $98.5(1.7)$ & $97.2(3.5)$ & 1000 \\
\hline & SWAT & $30-390-4$ & $93.1(2.3)$ & $91.5(2.3)$ & 500 \\
\hline & TMM-SNN & $30-(4-7)-4$ & $97.6(1.3)$ & $97.5(0.7)$ & 12 \\
\hline & OMLA & $30-(4-9)$ & $99.2(0.8)$ & $98.3(1.0)$ & 1 \\
\hline
\end{tabular}




\subsection{Summary}

In this chapter, the concept of meta-neuron has been presented. The idea of meta-neuron is inspired by the heterosynaptic plasticity induced by the astrocytes in biological neural networks. An astrocyte cell can connect to multiple synapses simultaneously, intercept the activity on the connected synapses and modulate the plasticity of these synapses.

In a spiking neural network with a meta-neuron, the meta-neuron is connected to all the input neurons. This allows the meta-neuron to access the presynaptic activity of the input neurons. Further, the meta-neuron can also access the synaptic weights in the network. Hence, the meta-neuron has access to the local information on a synapse as well as the global information stored in the network. The meta-neuron uses this information to compute the weight sensitivity modulation factor for the synapses in the network. The meta-neuron based learning rule uses the weight sensitivity modulation factor of a synapse and the postsynaptic potential of a neuron to update the synaptic weights in the network such that the spike time of the postsynaptic neuron is shifted precisely in the desired direction. This property of the meta-neuron based learning rule has been used to develop an online meta-neuron based learning algorithm.

The Online Meta-neuron based Learning Algorithm (OMLA) uses heuristic learning strategies to learn from the input spike patterns in a single presentation. It can choose to add a neuron, delete the sample or update the network parameters. The OMLA initializes the weights and threshold of a newly added neuron such that it fires at a precise time for the current input spike pattern. The OMLA uses a meta-neuron with memory that is used to store the previously learnt spike patterns used by OMLA to add a neuron to the network. The OMLA uses these spike patterns to update the synaptic weights of a newly added neuron so that it closely approximates the past knowledge stored in the network. The weights of the neurons are updated using the meta-neuron based learning rule.

The performance of the OMLA has been evaluated on ten benchmark problems from the UCI machine learning repository. The performance evaluation results have been statistically compared with that of other existing online learning algorithms using the one-way ANOVA test followed by a pairwise comparison using the Bonferroni test. The results of statistical comparison clearly indicate that OMLA performs better within a 95\% confidence interval. The performance evaluation results of OMLA have also been 
compared with other batch learning algorithms. The results of performance comparison clearly indicate that OMLA can achieve better performance using a compact network architecture. Further, OMLA requires a single epoch for learning whereas other algorithms require multiple epochs.

In the next chapter, the results of a preliminary study on the neuromorphic implementation of OMLA using an FPGA are presented. 
Chapter 5. OMLA 


\section{Chapter 6}

\section{Neuromorphic Implementation of the Online Meta-neuron based}

Learning Algorithm

The previous chapter presented the concept of meta-neuron in a Spiking Neural Network (SNN). A meta-neuron based learning rule, which utilized both the local and global information in the network, was proposed to update the synaptic weights. This learning rule was used to developed an Online Meta-neuron based Learning Algorithm (OMLA) for pattern classification problems.

The closeness of SNNs to biological neural networks motivated us to develop a neuromorphic implementation of the OMLA. This chapter presents results from a preliminary study on the hardware implementation of OMLA using a Field-Programmable Gate Array (FPGA). The performance of the described implementation is evaluated on ten benchmark problems from the UCI machine learning repository [21]. Also, a study is conducted using the Ionosphere problem from UCI machine learning repository to analyze the effect of reduced precision for storing weights and the effect of noise in the testing data on the performance of the proposed neuromorphic implementation. 


\subsection{Motivation}

Neuromorphic systems are hardware circuits that employ spikes for processing and transmitting information in a network. These systems consume power only when a spike is received or generated which enables them to operate for a longer period with minimal power consumption [94]. This has led to the development of several neuromorphic platforms for large scale simulations of Spiking Neural Networks (SNNs) [95, 96, 97, 98, 99, $100,101,102]$.

In $[95,103]$ a Spiking Neural Network Architecture (SpiNNaker) has been developed that consists of a 2-dimensional array of processing nodes each of which consists of 18 ARM processors. Different from SpiNNaker, which employs a digital approach, an analog approach has been used to model neurons and synapses in Heidelberg University BrainScaleS project [96]. The chip developed in the BrainScaleS project uses wafer-scale integration with analog circuits operating above threshold. This allows the chip to realize analog circuits that perform faster than real time. To leverage the advantages of analog as well as digital circuits, Neurogrid [97] and TrueNorth [98] employ a mixed analog-digital approach. Neurogrid operates analog circuits in a subthreshold regime; as a result, it can model a large number of neurons consuming a small amount of power. TrueNorth chip consists of 4096 cores, and each core can model 256 neurons. It has been developed to overcome the limitations imposed by the Von-Neumann architecture. Each core in TrueNorth has dedicated resources for memory and computations.

The neuromorphic platforms discussed above focus on modeling large scale spiking neural networks in hardware. These works do not address the issues of learning in SNNs. There are two major issues with regards to the development of learning algorithms for SNNs that are suitable for hardware realization. Firstly, signals in hardware use limited precision, unlike software simulations. The use of limited precision is important in hardware to ensure efficient utilization of resources. Secondly, processing in hardware is affected by noise due to device mismatch in circuits and random fluctuations in physical quantities like current $[104,105]$. Thus, there is a need to develop learning algorithms for SNNs that can robustly deal with reduced bit precision and noisy data.

Several neuromorphic systems that utilize either Spike-Driven Synaptic Plasticity (SDSP) [106] or contrastive divergence [107] have been proposed in literature. In [108, 
109], SDSP has been used to update the synaptic weights due to its easier hardware realizability. In [94, 110, 111], learning rules inspired by contrastive divergence have been used to update synaptic weights in a deep SNN. In [110], an on-chip contrastive divergence learning rule has been developed for training a network of spiking neurons. In [94], the synaptic weights of an offline trained deep belief network are mapped to a deep network of Siegert neurons [112]. A Siegert neuron is used as it closely approximates the mean firing rate of a leaky integrate-and-fire neuron.

Recently, the concept of morphological learning has been developed for updating the connectivity in the network instead of updating the synaptic weights $[113,114]$. For hardware realization, the SNN is trained offline and then the connection matrix is downloaded into hardware [115]. As a result, SNNs trained using morphological learning can be easily mapped to hardware circuits.

Most of the learning algorithms for neuromorphic systems described above train the network offline and then the learned weights are loaded into the hardware. The learning algorithms based on SDSP [108, 109] and contrastive divergence [110] realize on-chip learning. But, both SDSP and contrastive divergence are local learning mechanisms that do not take into account the global information in the network. To overcome this issue, this chapter describes a preliminary design for hardware realization of the online meta-neuron based learning algorithm on an FPGA device.

\subsection{Neuromorphic Implementation of the Online Meta- neuron Based Learning Algorithm}

In this section, the structure of the neuromorphic implementation for the Online Metaneuron based Learning Algorithm (OMLA) using an FPGA is described. Figure 6.1 shows the structure of the hardware realization of OMLA. The input bus is used to present spike patterns to the network. In the current design, each input neuron generates a single spike in the simulation interval. The time of spike is determined according to population coding scheme [17]. In a future design, the input bus will be replaced with an address event representation communication protocol to enable handling of spike trains. The predicted class label for the input spike pattern is latched onto the output bus. The 


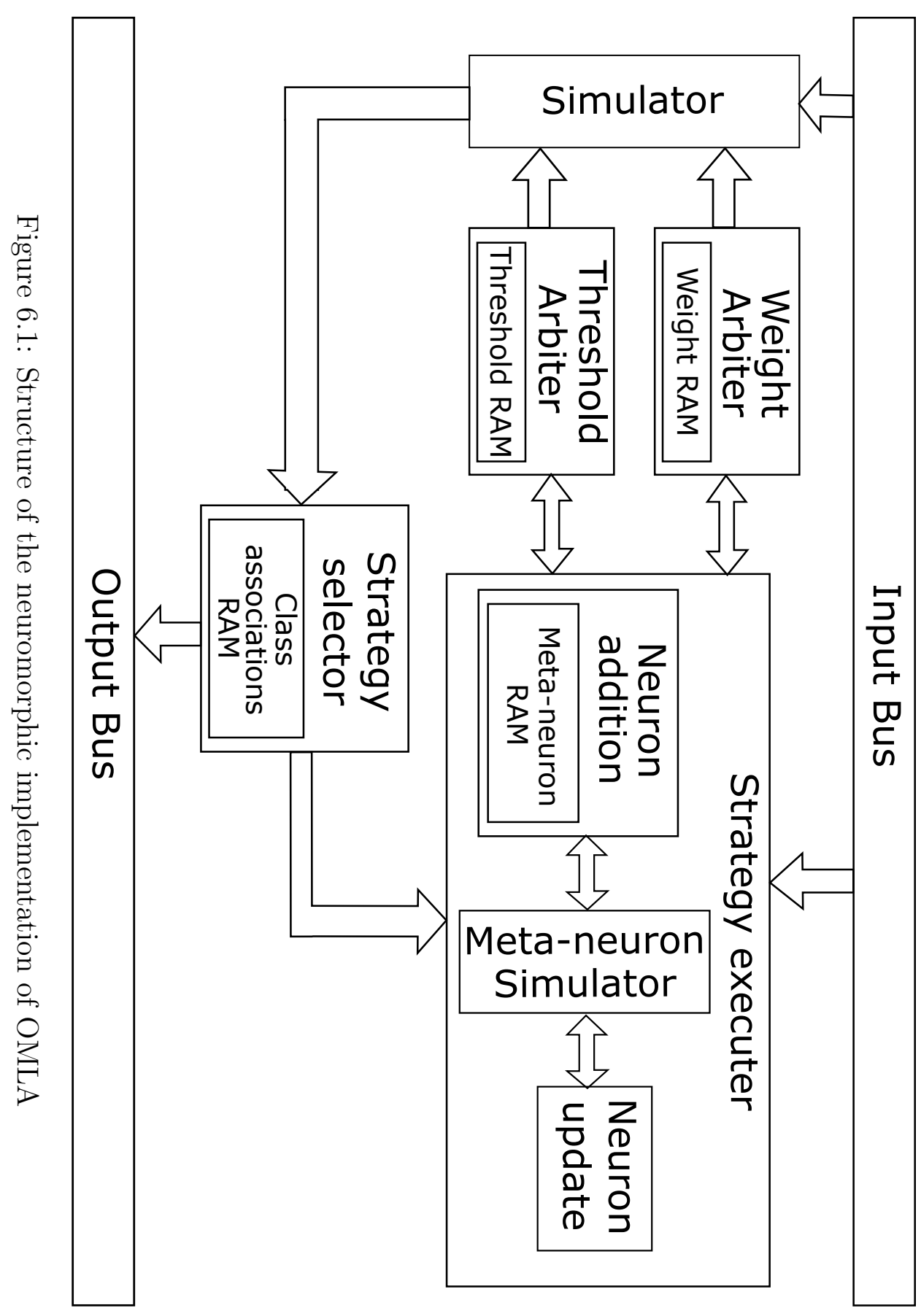


other main components of the design are simulator block, strategy selector block and strategy executer block. Next, each of the components will be described in detail.

Simulator block: The simulator block determines the response of the output neurons for a given input spike pattern. Figure 6.2 shows the structure of the simulator block with $m$ input neurons and $2^{K}$ output neurons. The response of each input neuron is the unweighted Postsynaptic Potential (PSP) induced by that neuron at a given time instant. The postsynaptic potential induced by the input neurons is passed to each output neuron which consists of a multiplier and an adder (shown in Figure 6.2). The multiplier is used to determine the weighted PSP induced by each input neuron and the adder is used to estimate the total PSP of that neuron at a given time instant.

The weights and threshold of each output neuron are stored in the 'weight RAM' and the 'threshold RAM', respectively (shown in Figure 6.1). The arbiters for the 'weight RAM' and the 'threshold RAM' control the access to the corresponding RAMs. During simulation, the simulator block has read access to the RAMs. Once the strategy selector block chooses a learning strategy for the current input spike pattern, the read access from the simulator block is withdrawn and then the strategy executer block is granted $\mathrm{read} /$ write access to the RAMs.

The total PSP of an output neuron is used by the comparator to determine whether the PSP is greater than the threshold for that neuron at a given time instant. The output of the comparator is connected to a neuron output register with $2^{K}$ bits. When the PSP of a neuron is greater than the threshold of that neuron, then the corresponding bit is set in the neuron output register. The neuron output register is connected to the strategy selector block, which determines the suitable learning strategy for a given input spike pattern.

The output neurons in the OMLA are modeled using the spike response function [17]. The unweighted PSP $(\epsilon()$.$) induced at time t$ by a presynaptic spike at $t_{\text {pre }}$ is given as

$$
\epsilon\left(t-t_{\text {pre }}\right)=\frac{t-t_{\text {pre }}}{\tau} \exp \left(1-\frac{t-t_{\text {pre }}}{\tau}\right)
$$

where $\tau$ is the time constant for that neuron. The time of all the presynaptic spikes (presented using the input bus) is scaled such that the time constant for the neuron 


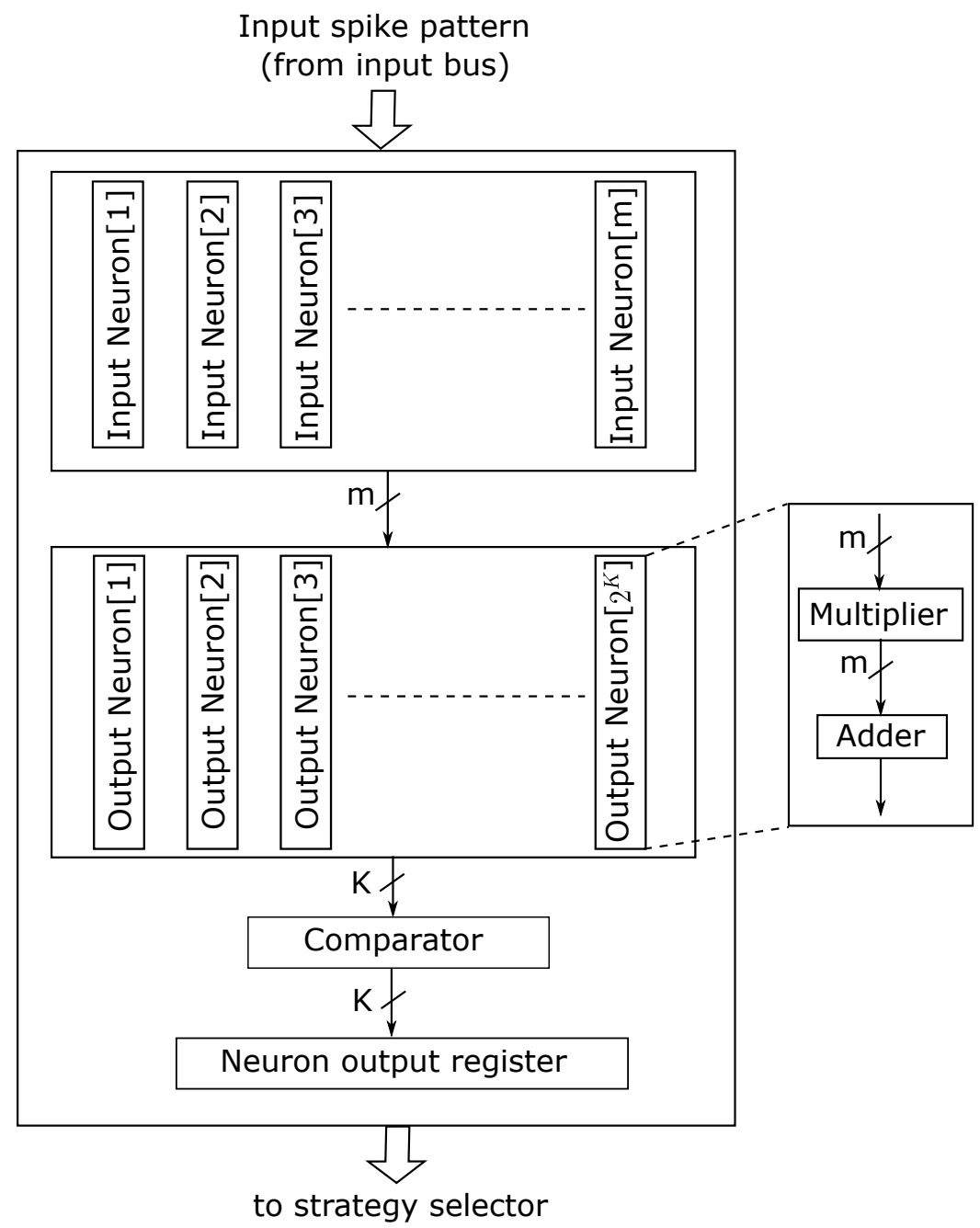

Figure 6.2: Structure of the simulator

can be set to one. This helps in avoiding the division operation in the calculation of PSP (Equation 6.1). The calculation of exponential in the spike response function is computationally the most expensive operation. The hyperbolic Coordinate Rotation Digital Computer (CORDIC) circuit [18] is used to calculate the value of the exponential operation. It uses shift and addition operations for multiple iterations and the number of iterations $(N)$ used, determine the accuracy of the final result. The CORDIC circuit is based on the iterative computation of the coordinates of a point in 2-dimension after rotation by a given angle. To compute $\exp (x)$, it updates the values of three variables $(w[e], \theta[e]$ and $d[e])$ in the $e^{t h}$ iteration. Here, $w[e]$ represents the value of $\exp (x)$ after $e^{t h}$ iteration, $\theta[e]$ represents the total rotation achieved after $i$ iterations and $d[e]$ represents 


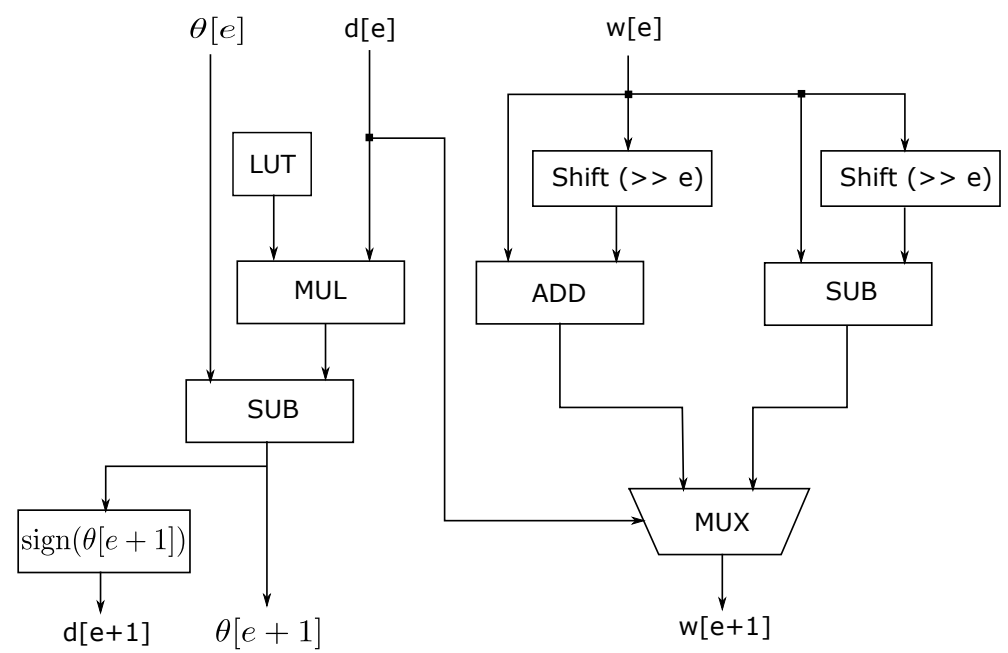

Figure 6.3: Single iteration of CORDIC computations

the direction of rotation in the $e^{t h}$ iteration. The values for $w[1], \theta[1]$ and $d[1]$ are initialized as follows:

$$
\begin{aligned}
& w[1]=\left(\prod_{e=1}^{N}\left(1-2^{2 e}\right) \prod_{e \in\{4, \cdots, l,(3 l+1), \cdots\}}^{N}\left(1-2^{2 e}\right)\right)^{-1 / 2} \\
& \theta[1]=x \\
& d[1]= \begin{cases}1 & \theta[1] \geq 0 \\
-1 & \theta[1]<0\end{cases}
\end{aligned}
$$

$w[1]$ depends on the number of iterations and is calculated beforehand. Given the values of $w[e], \theta[e]$ and $d[e]$ in the $e^{t h}$ iteration, their values in the $(e+1)^{t h}$ iteration are computed as follows:

$$
\begin{aligned}
& w[e+1]=w[e]+\left(w[e] * d[e] * 2^{-e}\right) \\
& \theta[e+1]=\theta[e]-\left(d[e] * \tanh ^{-1}\left(2^{-e}\right)\right) \\
& d[e+1]= \begin{cases}1 & \theta[e] \geq 0 \\
-1 & \theta[e]<0\end{cases}
\end{aligned}
$$

The values for the hyperbolic tangent function in Equation (6.6) are stored in a Lookup 


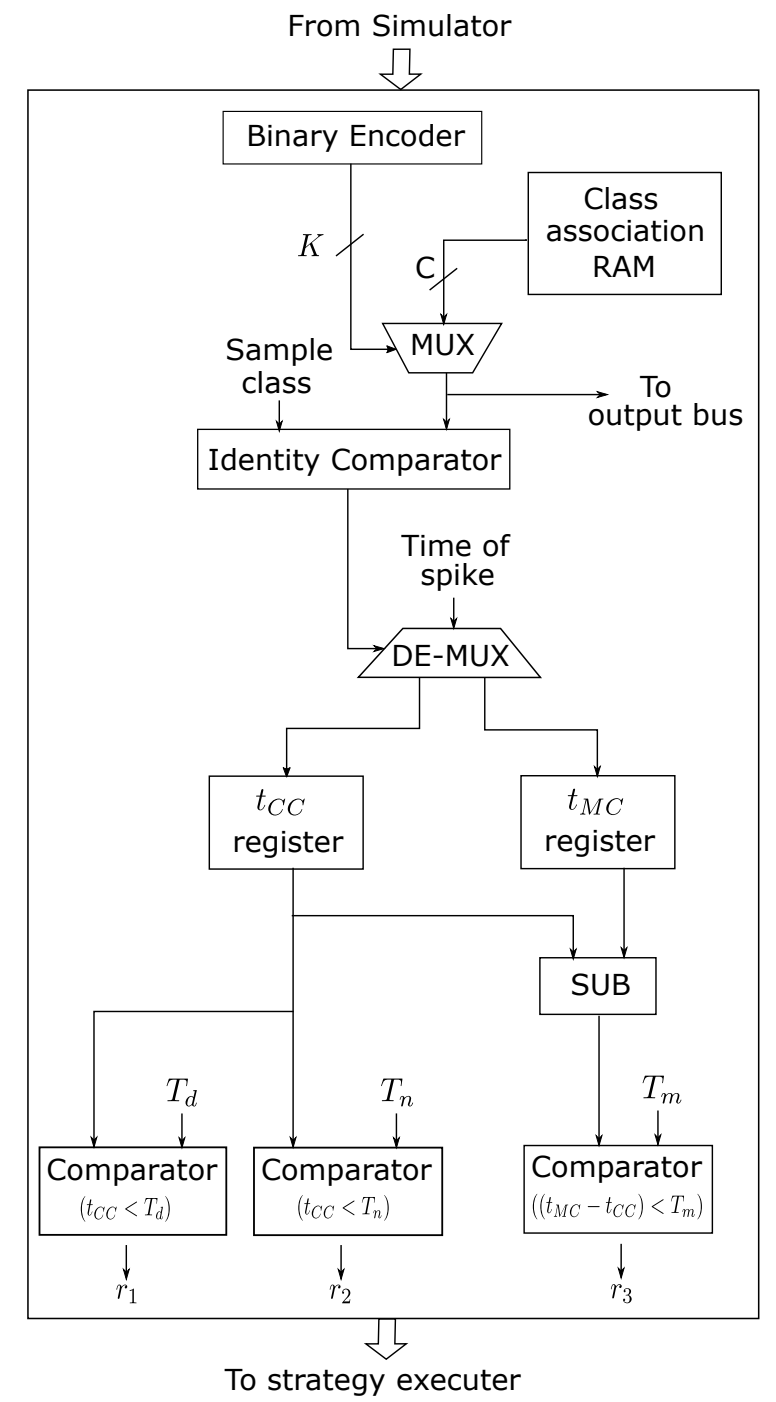

Figure 6.4: Structure of the strategy selector; this component selects the appropriate learning strategy for a given input spike pattern

Table (LUT) to minimize the FPGA resources consumed by the CORDIC circuit. Figure 6.3 shows the implementation of a single iteration of CORDIC calculations based on Equations 6.5, 6.6 and 6.7. The FPGA design of OMLA employs a pipelined CORDIC circuit that allows computation of the postsynaptic potential induced by a particular input neuron in a single clock cycle.

Strategy selector block: Figure 6.4 shows the structure of the strategy selector block. The strategy selector block consists of a class association RAM that is used to store the associated classes for the output neurons. The width of the memory locations in the class association RAM depends on the number of classes in a given problem. For example, 
Table 6.1: Truth table for strategy selection in strategy selector

\begin{tabular}{|c|c|c|c|}
\hline$r_{1}$ & $r_{2}$ & $r_{3}$ & Selected strategy \\
\hline 0 & 0 & $\mathrm{X}$ & Add a neuron \\
0 & 1 & 0 & Update the neuron $C C$ \\
0 & 1 & 1 & Update the neuron $C C$ and $M C$ \\
1 & $\mathrm{X}$ & 0 & Delete the sample \\
1 & $\mathrm{X}$ & 1 & Update the neuron $M C$ \\
\hline
\end{tabular}

the OMLA can handle problems with $2^{C}$ classes when the memory locations in the class association RAM are $C$ bits wide. The binary encoder in the strategy selector determines the address of the neuron that fired. During testing, the address of the neuron that fires first is used to retrieve the associated class for that neuron from the class association RAM. The associated class is placed on the output bus as the predicted class label for the current input spike pattern. In the training phase, the associated class is compared with the sample class using an identity comparator. If the class of the spike pattern is same as the predicted class label then the time of spike for the neuron is saved in the $t_{C C}$ register otherwise, the time of spike for the neuron is saved into the $t_{M C}$ register. $t_{C C}$ and $t_{M C}$ are used to determine the learning strategy for a given input spike pattern based on the outputs $r_{1}, r_{2}$ and $r_{3}$. Table 6.1 describes the truth table for selecting the learning strategy for a given input spike pattern. It may be noted that when the strategy selector decides to update the synaptic weights, it also determines the desired firing times for the neurons $C C$ and $M C$. The desired firing time $\left(\bar{t}_{C C}\right)$ for the neuron $C C$ is given by the Equation 5.28 and the desired firing time $\left(\bar{t}_{M C}\right)$ of the neuron $M C$ is given by the Equation 5.29.

Strategy executer block: The strategy executer consists of three modules namely 'meta-neuron simulator module', 'neuron addition module' and 'neuron update module'. The 'meta-neuron simulator module' is used by both the other modules to simulate the meta-neuron in the network. The 'neuron addition module' and the 'neuron update module' are used to execute the 'neuron addition strategy' and the 'parameter update strategy', respectively. Next, a description of these three components is provided.

- Meta-neuron simulator module: This module is used to initialize the weights of a newly added neuron and for updating the synaptic weights. Figure 6.5 shows the 


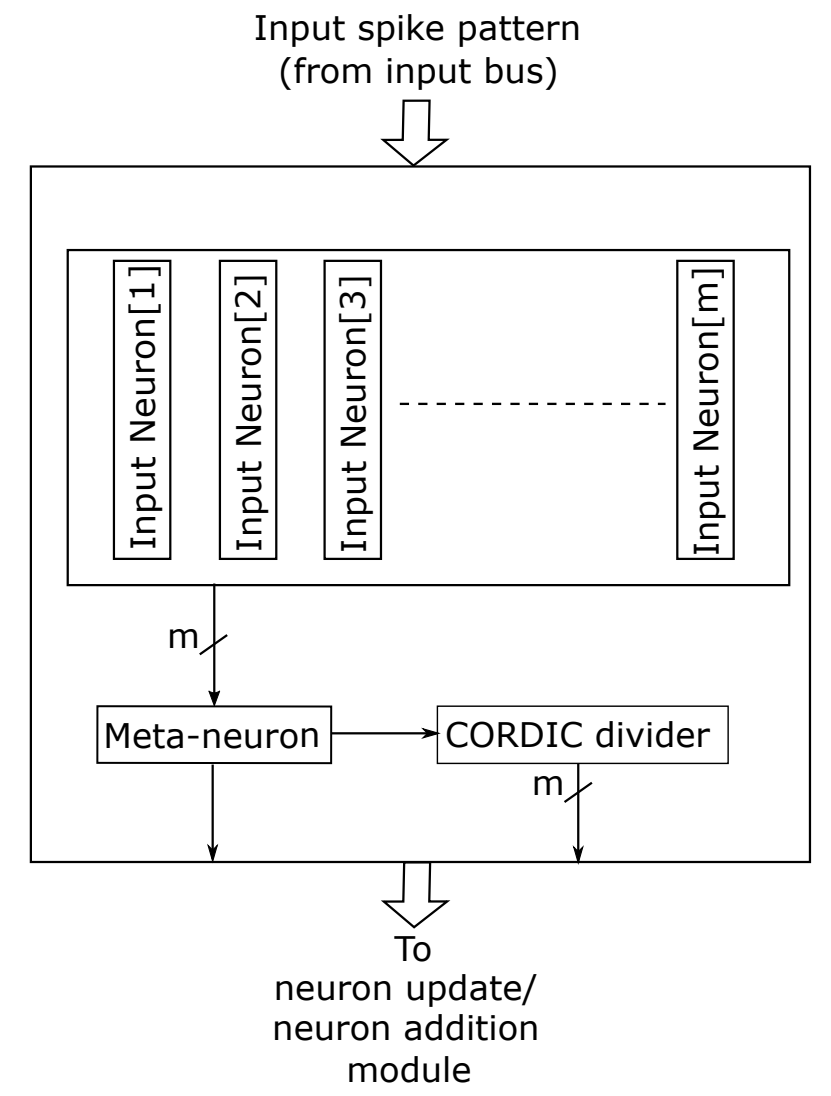

Figure 6.5: Structure of meta-neuron simulator module

structure of the meta-neuron simulator module. Similar to the simulator block, it employs a CORDIC circuit to compute the unweighted PSP induced by the input neurons at a given time instant. The structure of the meta-neuron is similar to the structure of the output neuron (shown in Figure 6.2) and its purpose is to compute the PSP of the meta-neuron.

The weights of the meta-neuron are initialized for each input spike pattern either by the neuron addition module or the neuron update module depending on the strategy chosen by the strategy selector block. The CORDIC divider in the metaneuron simulator module computes the ratios of the weighted PSP induced by the input neurons to the total PSP of the meta-neuron. It may be noted that the neuron addition module and the neuron update module can access the output of the meta-neuron as well as the output of the CORDIC divider. Next, the CORDIC circuit used to compute these ratios is described. 
Suppose $v^{i}(t)$ denotes the weighted PSP induced by the $i^{t h}$ input neuron at time $t$, then the ratios $\left(r_{i}\right)$ of the weighted PSP induced by the input neurons to the total PSP of the meta-neuron is given by

$$
r_{i}=\frac{v^{i}(t)}{\sum_{i} v^{i}(t)}
$$

The division operations are difficult to execute in hardware. The CORDIC circuit provides an iterative technique to compute division in hardware using only addition and shift operations. To compute $\frac{a}{b}$, the values of $w[1], \theta[1]$ and $d[1]$ are initialized as follows:

$$
\begin{aligned}
& w[1]=a \\
& \theta[1]=b \\
& d[1]= \begin{cases}1 & \theta[1] \geq 0 \\
-1 & \theta[1]<0\end{cases}
\end{aligned}
$$

Given the values of $w[e], \theta[e]$ and $d[e]$ in the $e^{t h}$ iteration, their values in the $(e+1)^{t h}$ iteration are computed as follows:

$$
\begin{aligned}
& w[e+1]=w[e]-\left(d[e] * \theta[e] * 2^{-e}\right) \\
& \theta[e+1]=\theta[e]+\left(d[e] * 2^{-e}\right) \\
& d[e+1]= \begin{cases}1 & w[e]>0 \\
-1 & w[e]<0\end{cases}
\end{aligned}
$$

It may be noted that in this case, the CORDIC circuit does not require any lookup table. The result of the division $\frac{a}{b}$ is obtained from $w[N]$.

- Neuron update module: This module updates the weights of the neurons $C C$ and $M C$ such that they fire at $\bar{t}_{C C}$ and $\bar{t}_{M C}$, respectively. For this purpose, it computes the required change in PSP of the corresponding neuron and the weight sensitivity modulation factor of all the synapses of the particular neuron. 
- To compute the required change in PSP, the neuron update module initializes the meta-neuron weights to the existing weights of the output neuron to be updated. Based on the PSP of the meta-neuron, the required change in PSP is given by the Equation 5.5.

- To compute the weight sensitivity modulation factor, the meta-neuron simulator module is presented with the current input spike pattern twice. In the first presentation, the neuron update module initializes the meta-neuron weights to one for computing the normalized PSP (Equation 5.8) of the input neurons at the desired time of spike. In the second presentation, the computed normalized PSP is used to initialize the weights of the meta-neuron, as defined in Equation 5.9. The output of the CORDIC divider in this presentation provides the weight sensitivity modulation factor for the synapses of the neuron to be updated.

- Neuron addition module: This module is used to initialize and update the weights of a newly added neuron such that it closely approximates the past information stored in the network. The weights of a newly added neuron are initialized as the normalized PSP induced by a particular output neuron that is computed as described above.

After initializing the weights of the newly added neuron, the neuron addition module uses the spike patterns stored in the meta-neuron RAM to update the weights of the newly added neuron as described above. The meta-neuron RAM stores the previous spike patterns that were used by the OMLA to add a neuron to the network along with their associated classes. The neuron addition module also presents the spike patterns stored in meta-neuron RAM to the meta-neuron simulator multiple times to update the weights of the newly added neuron.

Both the neuron addition module and the neuron update module present the input spike pattern to the meta-neuron simulator module multiple times to determine the updated weights of a given output neuron. These repeated presentations can be avoided by storing the PSP induced by the input neurons in a small interval around the time of 
spike. A future formulation of the meta-neuron based learning rule will focus on reducing the number of repeated presentations required for updating the synaptic weights.

In the next section, the design described above is evaluated for ten benchmark problems from the UCI machine learning repository [21]. Also, a study is conducted using the Ionosphere problem from UCI repository to analyze the performance of the learning algorithm for reduced bit precision and the effect of noise on the learning algorithm.

\subsection{Performance Evaluation}

In this section, the performance of OMLA with Reduced Bit Precision (OMLA-RBP) is evaluated on ten benchmark problems from the UCI machine learning repository [21]. The performance evaluation results of OMLA-RBP are compared with the performance of OMLA in software. The data sets from the benchmark problems are converted into spike times using the population coding scheme [17] as described in Section 3.1.1. The spike times determined using population coding scheme are presented to the FPGA implementation of OMLA.

The performance of OMLA-RBP is evaluated based on overall training and testing accuracy, which is equal to the percentage of the total number of samples that are correctly classified by the OMLA-RBP. All the experiments have been carried out in Xilinx Vivado 2016.1 using a CPU with 12 logical cores, 16 GB memory with a speed of $3.2 \mathrm{GHz}$. Before presenting the results of the performance evaluation, a study is conducted using the Ionosphere problem to analyze the effect of different bit precisions and noise on the performance of the OMLA-RBP.

Throughout this section, the notation $Q a . b$ is used to describe the fixed bit representation. Here, $a$ is the number of bits used to store the integer part including the sign bit and $b$ is the number of bits used to store the fractional part.

\subsubsection{Study on Performance of OMLA with Reduced Bit Pre- cision using Ionosphere Problem}

The Ionosphere problem has 351 samples that represent information about signals received by 16 high frequency antennas. The samples are classified into two classes based 


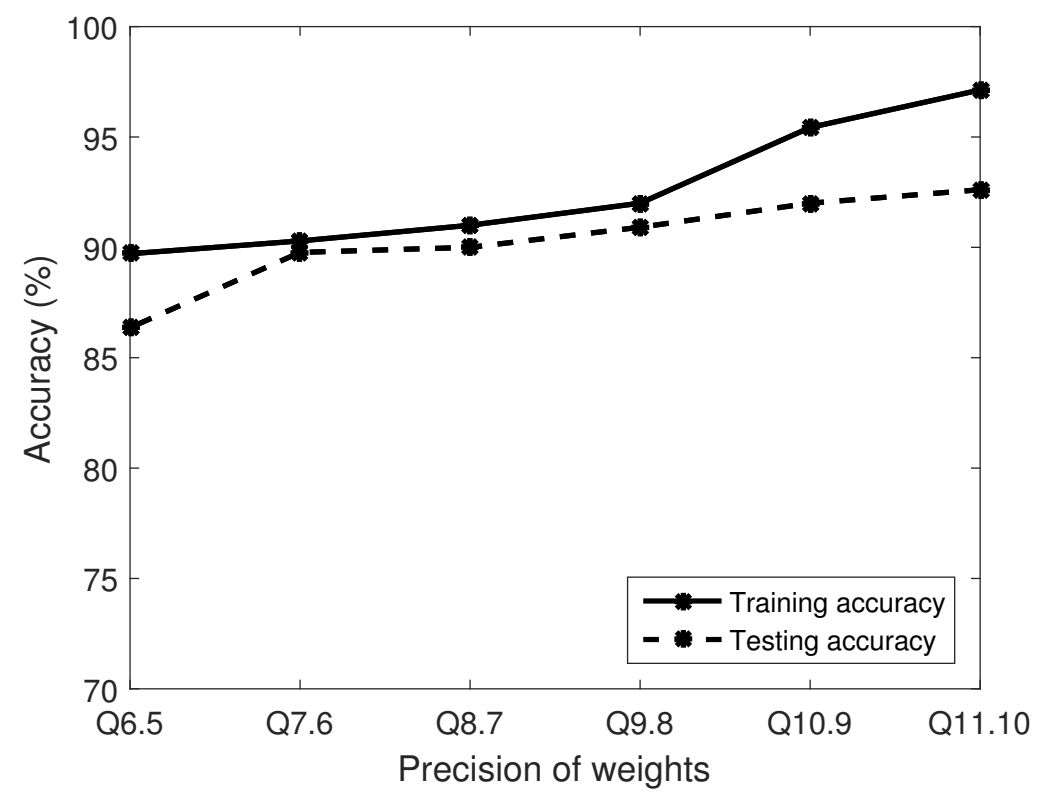

(a) Effect of precision on the classification accuracy of OMLA-RBP

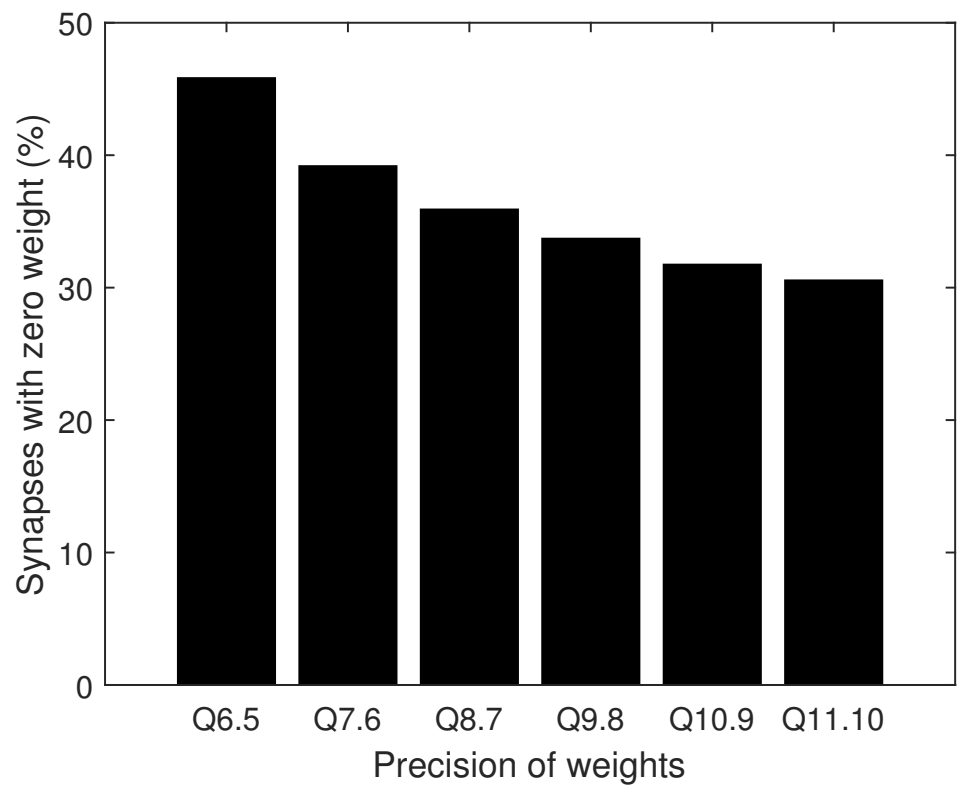

(b) Effect of precision on the number of synapses with zero weights

Figure 6.6: Effect of precision on the performance of OMLA-RBP

on whether they provide information about the structure of Ionosphere. For evaluating the fixed bit implementation of Online Meta-neuron based Learning Algorithm (OMLA), 175 samples are used for training and the rest of the samples are used for testing.

A reduced bit precision for synaptic weights results in lower utilization of resources. As 
a result, neural networks with higher number of neurons can be simulated with the same set of resources. To analyze the impact of reduced bit precision for synaptic weights on the performance of the OMLA, the performance of OMLA-RBP is evaluated for different precision values. Figure 6.6a shows the effect of precision on the performance of the OMLA-RBP. It can be observed that both training and testing accuracy decrease as the number of bits used to store weights are reduced. Figure $6.6 \mathrm{~b}$ shows the effect of precision on the percentage of zero weights in OMLA-RBP. It can be observed that the number of zero weights increase as the precision is reduced from $Q 11.10$ to Q6.5. The performance of OMLA-RBP goes down by just $7 \%$ while the percentage of zero-weights increase from $30 \%$ to almost $50 \%$. This indicates that loss in performance is smaller for reduced precision. Next, the effect of noise on the performance of the OMLA will be demonstrated.

Both analog and digital circuits are affected by noise due to variations in the circuit components and fluctuations in the physical quantities like current, voltage, etc. This makes robustness to noise an essential requirement for hardware realizability. To examine the noise sensitivity of OMLA, it is trained with noiseless data but tested on spike patterns with different levels of noise. The noise is introduced in the testing spike patterns in the form of additive white Gaussian noise. The performance of the OMLA-RBP is computed using a fixed-point representation of Q10.9 for storing synaptic weights. Figure 6.7 shows the testing accuracy of the OMLA for different values of the Signal-to-Noise Ratio (SNR). It can be observed from the table that the testing accuracy of the OMLA does not degrade significantly for SNR ratios between 20 to 40. For SNR ratios lower than 20, the testing accuracy for OMLA degrades rapidly. Thus, even for lower SNR of 20, OMLA can achieve acceptable performance.

\subsubsection{Comparison of OMLA-RBP and Software Simulations of OMLA}

In this section, the performance of OMLA with Reduced Bit Precision (OMLA-RBP) is evaluated on ten benchmark data sets from the UCI machine learning repository [21]. The results of OMLA-RBP are generated using a fixed point format of $Q 10.9$ for storing synaptic weights. The performance evaluation results are compared with the performance 


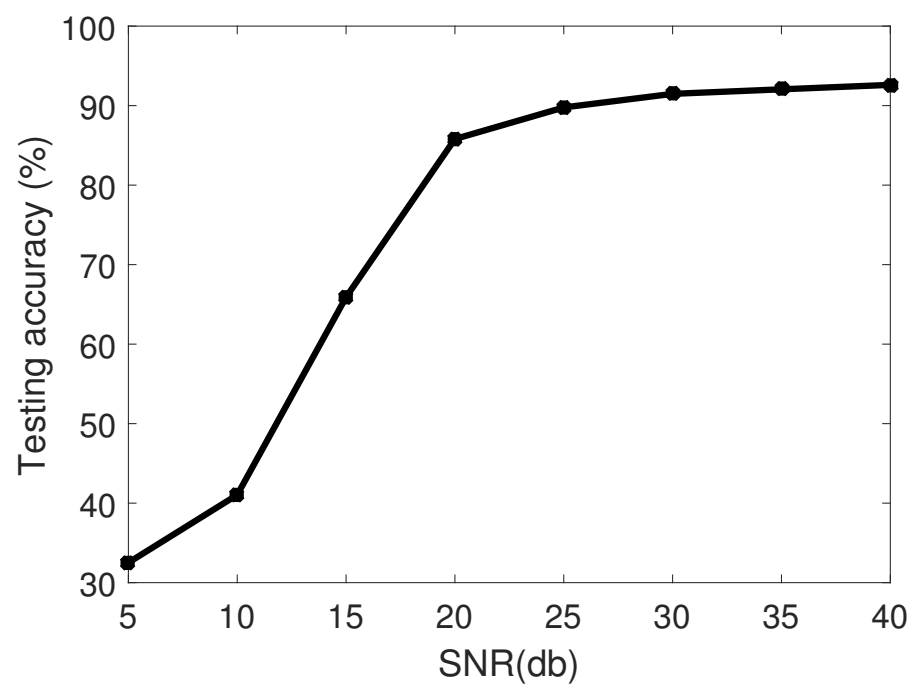

Figure 6.7: OMLA performance (\%) for different noise levels (SNR) for the Ionosphere problem

of OMLA obtained in software simulations. The software simulations use double precision for storing synaptic weights. As per IEEE 754 standard, the double precision uses a Q64.52 word length. The results for both OMLA-RBP and OMLA with double precision (OMLA-DP) are computed for ten random trials. For every data set, ten random sets are generated using the same number of training and testing samples (as described in [93]). The training and testing accuracy are computed for each set and the mean and standard deviation of the experiments are reported. The details of the data sets used for evaluation are provided in Table 5.1. The values of the parameters novelty threshold and update factor for the different data sets are provided in Table 5.2. Table 6.2 provides the results of performance comparison of OMLA-RBP and OMLA-DP.

It can be observed that for simple problems like Breast cancer, Iris, Wine and acoustic emission, the training and testing accuracies of OMLA-RBP is almost same as the performance of OMLA-DP. For all other problems except the Mammogram problem, the testing accuracy of OMLA-RBP is 2-3\% percent lower than the performance of OMLA-DP. In the case of Mammogram problem, OMLA-RBP performs 5\% lower than OMLA-DP. This can be attributed to a lower number of testing samples in case of the Mammogram problem. It has only 11 testing samples; as a result, a single misclassification result in a reduction of $9 \%$ in the testing accuracy. 
Table 6.2: Performance comparison of OMLA-RBP with OMLA-DP

\begin{tabular}{|c|c|c|c|c|}
\hline \multirow{2}{*}{ Data set } & \multicolumn{2}{|c|}{ OMLA-RBP } & \multicolumn{2}{c|}{ OMLA-DP } \\
\cline { 2 - 5 } & $\begin{array}{c}\text { Training } \\
\text { accuracy (\%) }\end{array}$ & $\begin{array}{c}\text { Testing } \\
\text { accuracy (\%) }\end{array}$ & $\begin{array}{c}\text { Training } \\
\text { accuracy (\%) }\end{array}$ & $\begin{array}{c}\text { Testing } \\
\text { accuracy (\%) }\end{array}$ \\
\hline Breast cancer & $96.9(0.5)$ & $97.5(0.8)$ & $97.4(0.4)$ & $97.8(0.4)$ \\
Iris & $98.2(0.6)$ & $97.3(1.1)$ & $97.9(0.7)$ & $97.9(0.7)$ \\
Wine & $99.4(0.8)$ & $96.6(1.8)$ & $98.5(1.0)$ & $97.9(0.7)$ \\
Acoustic Emission & $98.9(0.8)$ & $97.3(1.8)$ & $99.2(0.8)$ & $98.3(1.0)$ \\
Echocardiogram & $88.2(2.0)$ & $84.0(1.6)$ & $89.6(1.5)$ & $86.3(0.9)$ \\
Mammogram & $86.7(5.0)$ & $80.2(4.2)$ & $88.6(2.0)$ & $85.5(4.1)$ \\
Liver & $70.3(1.9)$ & $65.7(1.0)$ & $69.9(2.3))$ & $67.7(1.8))$ \\
PIMA & $78.3(2.3)$ & $75.4(1.7)$ & $78.6(1.7)$ & $77.9(1.0)$ \\
Ionosphere & $92.87(1.6)$ & $91.2(0.8)$ & $94.0(1.7)$ & $93.5(0.5)$ \\
Hepatitis & $86.1(4.3)$ & $84.1(5.6)$ & $89.8(2.9)$ & $87.4(2.4)$ \\
\hline
\end{tabular}

The performance comparison clearly indicates that the performance of OMLA-RBP is similar to that of OMLA-DP.

\subsection{Summary}

This chapter presents the results of a preliminary study on hardware realization of the OMLA. The spiking neurons in the hardware are modeled using the spike response function [17]. A CORDIC circuit, which only requires addition and shift operations, is used to estimate the potential induced by the input neurons. A study is conducted using the Ionosphere problem from the UCI machine learning repository to analyze the impact of reduced bit precision for synaptic weights and noise on the performance of the OMLA. The results of the study show that the OMLA can achieve acceptable performance even when only 6 bits are used to store synaptic weights. The results of the study also highlight that its performance does not deteriorate significantly when the testing spike patterns are subject to a lower signal-to-noise ratio of 20 . The hardware implementation of OMLA is also evaluated on ten benchmark problems from the UCI machine learning repository. The performance evaluation results of OMLA with reduced bit precision have been compared with the performance of OMLA in software simulations. The performance comparison indicates that the OMLA with reduced bit precision is able to emulate the software simulations of OMLA. 
Chapter 6. Neuromorphic Implementation of OMLA 


\section{Chapter 7}

\section{Conclusions and Future Works}

\subsection{Conclusions}

The aim of this thesis is to develop efficient batch/online learning algorithms for Spiking Neural Networks (SNNs) that can simultaneously update and evolve the network architecture. For this purpose, the works presented in this thesis are the Self-Regulating Evolving Spiking Neural (SRESN) classifier, the Two stage Margin Maximization Spiking Neural Network (TMM-SNN), the meta-neuron based learning rule and the Online Meta-neuron based Learning Algorithm (OMLA).

The SRESN classifier uses a batch learning technique to estimate the synaptic weights in a two layered SNN. It uses heuristic conditions to evolve the network architecture and update the synaptic weights. Depending on the response of the network for a given sample, it can choose to add a neuron, update the synaptic weights or skip learning the sample from the learning process. The weights and thresholds of the newly added neurons are initialized such that they fire precisely for the current input spike pattern. The SRESN classifier uses rank order learning in a feature-wise manner to initialize and update the synaptic weights. This helps the SRESN classifier to avoid non-contributing weights. Further, the SRESN classifier updates the weights selectively which helps in avoiding over-fitting and prevents loss of knowledge gained from previously learned samples.

The TMM-SNN uses a batch learning technique for a three layered SNN. The learning algorithm of TMM-SNN has two stages namely, structure learning stage and output weights learning stage. In the structure learning stage, the learning algorithm evolves the 
hidden layer and updates the synaptic weights of the hidden neurons. A new activation based coding scheme has been developed in this thesis to initialize the parameters (weights and threshold) of a newly added neuron. The activation based coding scheme utilizes only the locally available information present in multiple spikes to initialize the weights of a newly added neuron such that it spikes at a precise time. The weights of the hidden neurons are updated using a new margin maximization technique. This technique uses only the locally available information to update the weights of hidden neurons such that the separation between the spike times of the interclass neurons in the hidden layer is maximized for all training spike patterns. In the output weights learning stage, the learning algorithm updates the synaptic weights of the output layer neurons using a similar margin maximization technique to maximize the separation between the spike times of the interclass output layer neurons.

The performance of TMM-SNN has been evaluated on ten benchmark data sets from the UCI machine learning repository [21]. The performance evaluation results have been statistically compared with that of the SpikeProp [17], synaptic weight association training [10] and the SRESN classifier using the one-way ANOVA test [22] followed by a pairwise comparison using the Fisher's least significant difference method [23]. The results of statistical comparison clearly indicate that TMM-SNN performs better than the other learning algorithms in a 95\% confidence interval. This shows that TMM-SNN effectively utilizes the locally available information to update the synaptic weights. Further, the activation based coding scheme and the margin maximization update mechanism help the TMM-SNN in achieving improved generalization performance and faster convergence.

The meta-neuron based learning rule has been developed for updating synaptic weights in a two layered SNN with a meta-neuron. The concept of meta-neuron is inspired by the heterosynaptic plasticity exhibited by astrocytes. The meta-neuron based learning rule utilizes the local as well as global information in the network to update the synaptic weights of a neuron such that its spike time is shifted precisely in the desired direction. This property of the meta-neuron based learning rule is used to develop an Online Metaneuron based Learning Algorithm (OMLA) that requires a single presentation of the input spike patterns for learning.

The OMLA uses heuristic conditions to evolve and update the network architecture. 
It can choose to add a neuron or update the network parameters or delete a spike pattern from the learning process. The OMLA uses a meta-neuron with memory to update the synaptic weights of a newly added neuron such that it properly approximates the past knowledge stored in the network. It uses the meta-neuron based learning rule to update the synaptic weights of a neuron.

The performance of the OMLA has been evaluated on ten benchmark problems from the UCI machine learning repository. The performance evaluation results have been statistically compared with that of other online learning algorithms including online SNN proposed in [81] and the SRESN classifier trained using a high learning rate for five benchmark problems. The statistical comparison has been conducted using one-way ANOVA test [22] followed by a pairwise comparison using the Bonferroni test $[25,26]$. The results of statistical comparison clearly indicate that OMLA performs better than the other algorithms within a $95 \%$ confidence interval. The performance evaluation results of OMLA are also compared with other batch learning algorithms for ten benchmark problems. The performance comparison clearly indicates that OMLA performs better than the other algorithms using a single epoch of learning. Further, OMLA achieves these results using a compact network structure in comparison to the other learning algorithms. These results clearly indicate that the meta-neuron based learning rule effectively utilizes the local and global information in the network to update the synaptic weights.

This thesis also presents the results of a preliminary study on the realization of the OMLA in a Field-Programmable Gate Array. The spiking neurons in the design are modeled using the spike response function [17]. A study is conducted using the ionosphere problem from the UCI machine learning repository to analyze the effects of reduced bit precision for synaptic weights and noise on the performance of the hardware realization of OMLA. The FPGA realization of the OMLA with reduced bit precision for synaptic weights is also evaluated on ten benchmark problems from the UCI machine learning repository. The results of performance comparison show that the performance of OMLA with reduced bit precision is similar to the performance of OMLA in software simulations. 


\section{$7.2 \quad$ Future Directions}

The techniques explained in this thesis have focused on the development of efficient evolving learning algorithms for SNNs. The possible future directions based on the presented ideas are:

\subsubsection{Encoding Real Valued Features Into Spike Trains}

The popular encoding techniques like population coding [17] and cosine coding [83] employ multiple neurons to encode real valued data into spike patterns and each neuron generates a single spike in the simulation interval. But, biological neurons use spike trains to represent information in the brain. To gain better insights into the information processing mechanisms in the brain, it is important to develop techniques that utilize multiple spikes for encoding real valued data into spike patterns. This may also help in learning with smaller network architectures.

\subsubsection{Improvements in the Meta-neuron Based Learning Rule}

The meta-neuron based learning rule can be extended in future works in the following directions:

\subsubsection{Meta-neuron Based Learning Rule for Multilayer Spiking Neural Networks}

The meta-neuron based learning rule has been developed for estimating the synaptic weights in a two layered SNN. The learning rule updates the synaptic weights in the network based on the difference between the actual and the desired times of spikes. It is difficult to define the desired spike times for hidden neurons in a multilayer SNN. As a result, the meta-neuron learning rule can not be used for updating the hidden neuron weights in a multilayer SNN. Future work can focus on formulating the meta-neuron based learning rule such that it could be used for estimating network parameters in a multilayer SNN. 


\subsubsection{Computational Complexity}

The meta-neuron based learning rule has been developed to update the weights of a single neuron. As a result, the meta-neuron is presented with the current input spike pattern multiple times to update the weights of multiple neurons. This significantly increases the computational complexity of the meta-neuron based learning rule. In future work, the formulation of the meta-neuron based learning rule can be improved to update the weights of multiple neurons simultaneously.

\subsubsection{Understanding the Astrocytes induced Plasticity}

The idea of synaptic plasticity induced by astrocytes is based on very recent works in the area of neuroscience [14]. As a result, our understanding of this plasticity mechanism exhibited by the cells in the brain is very limited. This idea inspired the meta-neuron based learning rule, but it has been developed with the focus on addressing machine learning problems. There are substantial differences between the meta-neuron based learning rule and the physiology of the astrocyte cells in the brain. Future work can be conducted on the meta-neuron based learning rule with focus on studying the role of astrocytes in the brain.

\subsubsection{Address Event Representation (AER) Based Communi- cation Protocol}

The hardware implementation of the Online Meta-neuron based Learning Algorithm (OMLA) employs a $2^{K}$ bit register to detect the spikes generated by the input neurons, where $2^{K}$ is the number of neurons in the output layer. This results in considerable memory overhead to simulate large number of neurons, thereby limiting the application of OMLA for simpler problems requiring fewer neurons. A hardware implementation for OMLA can be developed in future that uses an AER based hardware system to simulate large number of neurons simultaneously. 


\subsubsection{Extension to Regression Problems}

The algorithms proposed in this thesis can also be used for addressing regression problems. But, the absence of a suitable technique that can map the response of spiking neurons to a real-valued continuous range limits the application of existing learning algorithms for SNNs to regression problems. For example, the meta-neuron based learning rule is used to train a network to generate multiple spikes at precise times irrespective of how the responses of output neurons are used to deduce predicted classes. This will be an important future direction for the work done in this thesis. 


\section{Author's Publications}

\section{Journals}

- S. Dora, K. Subramanian, S. Suresh and N. Sundararajan, Development of a self regulating evolving spiking neural network for classification problems, Neurocomputing, 2015.

- S. Dora, S. Suresh and N. Sundararajan, Sequential learning algorithm for a spiking neural classifier, Applied Soft Computing, 2015.

- S. Dora, S. Suresh and N. Sundararajan, Online Meta-neuron based Learning Algorithm for a Spiking Neural Classifier, Neural Computation, 2016 (in submission).

- S. Dora, S. Suresh and N. Sundararajan, A Two stage Margin Maximization Spiking Neural Network for Pattern Classification Problems, IEEE Transactions on Neural Networks and Learning Systems, 2016 (in submission).

\section{Conference Proceedings}

- S. Dora, R. Savitha and S. Suresh, A basis coupled evolving spiking neural network with afferent input neurons, in Proceedings of International Joint Conference on Neural Networks, 2013.

- S. Dora, S. Suresh and N. Sundararajan, A sequential learning algorithm for a minimal spiking neural network (MSNN) classifier, in Proceedings of International Joint Conference on Neural Networks, 2014. 
- S. Dora, S. Suresh and N. Sundararajan, A two-stage learning algorithm for a growing-pruning spiking neural network for a pattern classification problem, in Proceedings of International Joint Conference on Neural Networks, 2015.

- S. Dora, B. Rangarajan, K. Subramanian and S. Suresh, Automatic seizure detection in multichannel EEG using McCIT2FIS approach, in Proceedings of IEEE International Conference on Fuzzy Systems, 2015. 


\section{Bibliography}

[1] E. D. Adrian, "The impulses produced by sensory nerve-endings: Part ii: The response of a single end organ," The Journal of Physiology, vol. 62, no. 1, pp. 33-51, 1926.

[2] R. V. Rullen and S. J. Thorpe, "Rate coding versus temporal order coding: what the retinal ganglion cells tell the visual cortex," Neural Computation, vol. 13, no. 6, pp. 1255-1283, 2001.

[3] R. S. Johansson and I. Birznieks, "First spikes in ensembles of human tactile afferents code complex spatial fingertip events," Nature neuroscience, vol. 7, no. 2, pp. 170-177, 2004.

[4] T. Sasaki, N. Matsuki, and Y. Ikegaya, "Action-potential modulation during axonal conduction," Science, vol. 331, no. 6017, pp. 599-601, 2011.

[5] A. L. Hodgkin and A. F. Huxley, "A quantitative description of membrane current and its application to conduction and excitation in nerve," The Journal of physiology, vol. 117, no. 4, pp. 500-544, 1952.

[6] W. Gerstner and W. M. Kistler, Spiking Neuron Models: Single neuron, Populations and Plasticity. Cambridge University Press, 2002.

[7] E. M. Izhikevich, "Simple model of spiking neurons," IEEE Transaction on Neural Networks, vol. 14, no. 6, pp. 1569-1572, 2003.

[8] H. Markram, J. Lübke, M. Frotscher, and B. Sakmann, "Regulation of synaptic efficacy by coincidence of postsynaptic APs and EPSPs," Science, vol. 275, no. 5297, pp. 213-215, 1997. 
[9] J. Goldberg, K. Holthoff, and R. Yuste, "A problem with hebb and local spikes," Trends in neurosciences, vol. 25, no. 9, pp. 433-435, 2002.

[10] J. J. Wade, L. J. McDaid, J. A. Santos, and H. M. Sayers, "SWAT: A Spiking Neural Network Training Algorithm for Classification Problems," IEEE Transactions on Neural Networks, vol. 21, no. 11, pp. 1817-1830, 2010.

[11] K. Dhoble, N. Nuntalid, G. Indiveri, and N. Kasabov, "Online spatio-temporal pattern recognition with evolving spiking neural networks utilising address event representations, rank order, and temporal spike learning," in International Joint Conference on Neural Networks, 2012, pp. 1-7.

[12] N. Kasabov, K. Dhoble, N. Nuntalid, and G. Indiveri, "Dynamic evolving spiking neural networks for on-line spatio-and spectro-temporal pattern recognition," Neural Networks, vol. 41, pp. 188-201, 2013.

[13] D. J. Rossi, "Astrocytes join the plasticity party," Nature neuroscience, vol. 15, no. 5, pp. 649-651, 2012.

[14] M. D. Pittà, N. Brunel, and A. Volterra, "Astrocytes: orchestrating synaptic plasticity?" Neuroscience, vol. 23, pp. 43-61, 2015.

[15] R. D. Fields, A. Araque, H. Johansen-Berg, S.-S. Lim, G. Lynch, K.-A. Nave, M. Nedergaard, R. Perez, T. Sejnowski, and H. Wake, "Glial biology in learning and cognition," The Neuroscientist, vol. 20, no. 5, pp. 426-431, 2014.

[16] G. Perea and A. Araque, "Astrocytes potentiate transmitter release at single hippocampal synapses," Science, vol. 317, no. 5841, pp. 1083-1086, 2007.

[17] S. M. Bohte, J. N. Kok, and H. L. Poutré, "Error-backpropagation in temporally encoded networks of spiking neurons," Neurocomputing, vol. 48, no. 1-4, pp. 17-37, 2002.

[18] J. E. Volder, "The cordic trigonometric computing technique," IRE Transactions on Electronic Computers, vol. EC-8, no. 3, pp. 330-334, 1959. 
[19] S. Ghosh-Dastidar and H. Adeli, "A new supervised learning algorithm for multiple spiking neural network with application in epilepsy and seizure detection," Neural Networks, vol. 22, no. 10, pp. 1419-1431, 2009.

[20] Y. Xu, X. Zeng, L. Han, and J. Yang, "A supervised multi-spike learning algorithm based on gradient descent for spiking neural networks," Neural Networks, vol. 43, pp. 99-113, 2013.

[21] M. Lichman, "UCI Machine Learning Repository," 2013.

[22] R. A. Fisher, "On the probable error of a coefficient of correlation deduced from a small sample," Metron, vol. 1, no. 4, pp. 3-32, 1921.

[23] — The design of experiments. Oliver and Boyd Edinburgh, 1935.

[24] X. Xie, H. Qu, Z. Yi, and J. Kurths, "Efficient training of supervised spiking neural network via accurate synaptic-efficiency adjustment method," IEEE Transaction on Neural Networks and Learning Systems, 2016. doi: 10.1109/TNNLS.2016.2541339

[25] O. J. Dunn, "Estimation of the medians for dependent variables," The Annals of Mathematical Statistics, vol. 30, no. 1, pp. 192-197, 1959.

[26] — "Multiple comparisons among means," Journal of the American Statistical Association, vol. 56, no. 293, pp. 52-64, 1961.

[27] W. Maass, "Networks of spiking neurons: the third generation of neural network models," Neural Networks, vol. 10, no. 9, pp. 1659-1671, 1997.

[28] — - "Fast sigmoidal networks via spiking neurons," Neural Computation, vol. 9, no. 2, pp. 279-304, 1997.

[29] — - "Noisy spiking neurons with temporal coding have more computational power than signoidal neurons," Institute for Theoretical Computer Science, Technische Universitaet Graz, Austria, Technical Report, 1999. [Online]. Available: http://www.igi.tugraz.at/psfiles/90.pdf

[30] E. M. Izhikevich, "Polychronization: computation with spikes," Neural computation, vol. 18, no. 2, pp. 245-282, 2006. 
[31] N. K. Kasabov, "Neucube: A spiking neural network architecture for mapping, learning and understanding of spatio-temporal brain data," Neural Networks, vol. 52 , pp. $62-76,2014$.

[32] R. M. Wang, G. Cohen, K. M. Stiefel, T. J. Hamilton, J. C. Tapson, and A. V. Schaik, "An FPGA implementation of a polychronous spiking neural network with delay adaptation," Frontiers in Neuroscience, vol. 7, no. 14, 2013.

[33] G. Bi and M. Poo, "Synaptic modifications in cultured hippocampal neurons: dependence on spike timing, synaptic strength, and postsynaptic cell type," The Journal of neuroscience, vol. 18, no. 24, pp. $10464-40472,1998$.

[34] A. Mohemmed, S. Schliebs, S. Matsuda, and N. Kasabov, "SPAN: Spike pattern association neuron for learning spatio-temporal spike patterns," International Journal of Neural Systems, vol. 22, no. 04, 2012. doi: 10.1142/S0129065712500128

[35] Q. Yu, H. Tang, K. C. Tan, and H. Li, "Precise-spike-driven synaptic plasticity: Learning hetero-association of spatiotemporal spike patterns," PLoS ONE, vol. 8, no. 11, pp. 1-16, 2013. doi: 10.1371/journal.pone.0078318

[36] S. B. Shrestha and Q. Song, "Adaptive learning rate of SpikeProp based on weight convergence analysis," Neural Networks, vol. 63, pp. 185-198, 2015.

[37] J. Xin and M. J. Embrechts, "Supervised learning in spiking neural networks," in International Joint Conference on Neural Networks, vol. 3, 2001, pp. 1772-1777.

[38] S. McKennoch, D. Liu, and L. G. Bushnell, "Fast modifications of the spikspike algorithm," in International Joint Conference on Neural Networks, 2006, pp. 39703977.

[39] H. Takase, M. Fujita, H. Kawanaka, S. T. nad Hajime Kita, and T. Hayashi, "Obstacle to training spikeprop networks-cause of surges in training process," in International Joint Conference on Neural Networks, 2009, pp. 3062-3066.

[40] S. Ghosh-Dastidar and H. Adeli, "Improved spiking neural network for EEG classification and epilepsy and seizure detection," Integrated Computer-Aided Engineering, vol. 14, no. 3, pp. 187-212, 2007. 
[41] R. V. Florian, "The chronotron: A neuron that learns to fire temporally precise spike patterns," PLoS ONE, vol. 7, no. 8, pp. 1-27, 2012.

[42] J. D. Victor and K. P. Purpura, "Nature and precision of temporal coding in visual cortex:a metric space analysis," Journal of Neurophysiology, vol. 76, no. 2, pp. 1310-1326, 1996.

[43] R. Gütig and H. Sompolinsky, "The tempotron: a neuron that learns spike timing based decisions," Nature neuroscience, vol. 9, no. 3, pp. 420-428, 2006.

[44] H. Tang, Q. Yu, and K. C. Tan, "Learning real-world stimuli by single-spike coding and tempotron rule," in International Joint Conference on Neural Networks, 2012, pp. 1-6.

[45] Q. Yu, H. Tang, K. C. Tan, and H. Li, "Rapid feedforward computation by temporal encoding and learning with spiking neurons," IEEE Transactions on Neural Networks and Learning Systems, vol. 24, no. 10, pp. 1539-1552, 2013.

[46] Q. Yu, H. Tang, K. C. Tan, and H. Yu, "A brain-inspired spiking neural network model with temporal encoding and learning," Neurocomputing, vol. 138, pp. 3-13, 2014.

[47] F. Ponulak and A. Kasiński, "Supervised learning in spiking neural networks with resume: Sequence learning, classification and spike shifting," Neural Computation, vol. 22 , no. 2, pp. 467-510, 2010.

[48] A. Kasiński and F. Ponulak, "Experimental demonstration of learning properties of a new supervised learning method for the spiking neural networks," in Artificial Neural Networks: Biological Inspirations-ICANN 2005. Springer, 2005, pp. 145152.

[49] F. Ponulak, "Analysis of the ReSuMe learning process for spiking neural networks," International Journal of Applied Mathematics and Computer Science, vol. 18, no. 2, pp. 117-127, 2008. 
[50] W. M. Kistler and J. L. V. Hemmen, "Modelling synaptic plasticity in conjunction with the timing of pre- and postsynaptic action potentials," Neural Computation, vol. 12 , no. 2, pp. 385-405, 2000.

[51] I. Sporea and A. Grüning, "Supervised learning in multilayer spiking neural networks," Neural Computation, vol. 25, no. 2, pp. 473-509, 2013.

[52] A. Taherkhani, A. Belatreche, Y. Li, and L. P. Maguire, "DL-ReSuMe: A delay learning-based remote supervised method for spiking neurons," IEEE Transactions on Neural Networks and Learning Systems, vol. 26, no. 12, pp. 3137-3149, 2015.

[53] — - "EDL: an extended delay learning based remote supervised method for spiking neurons," in Neural Information Processing, 2015, pp. 190-197.

[54] —, "Multi-dl-resume: Multiple neurons delay learning remote supervised method," in International Joint Conference on Neural Networks, 2015, pp. 1-7.

[55] E. L. Bienenstock, L. N. Cooper, and P. W. Munro, "Theory for the development of neuron selectivity: orientation specificity and binocular interaction in visual cortex," The Journal of neuroscience, vol. 2, no. 1, pp. 32-48, 1982.

[56] J. Wang, A. Belatreche, L. Maguire, and T. M. McGinnity, "Dynamically evolving spiking neural network for pattern recognition," in International Joint Conference on Neural Networks, 2015, pp. 1-8.

[57] S. Thorpe and J. Gautrais, "Rank order coding," in Computational Neuroscience. Springer, 1998, pp. 113-118.

[58] S. G. Wysoski, L. Benushkova, and N. Kasabov, "Online learning with structural adaptation in a network of spiking neurons for visual pattern recognition," in International Conference on Artificial Neural Networks, 2006, pp. 61-70.

[59] S. G. Wysoski, L. Benuskova, and N. Kasabov, "Fast and adaptive network of spiking neurons for multi-view visual pattern recognition," Neurocomputing, vol. 71, no. 13, pp. 2563-2575, 2008. 
[60] —_ "Evolving spiking neural networks for audiovisual information processing," Neural Networks, vol. 23, no. 7, pp. 819-835, 2010.

[61] J. Wang, A. Belatreche, L. P. Maguire, and T. M. McGinnity, "Spiketemp: An enhance rank-order-based learning approach for spiking neural networks with adaptive structure," IEEE Transaction on Neural Networks and Learning Systems, 2015. doi: 10.1109/TNNLS.2015.2501322

[62] A. Mohemmed, S. Schliebs, and N. Kasabov, "SPAN: a neuron for precise-time spike pattern association," in International Conference on Neural Information Processing, 2011, pp. 718-725.

[63] A. Mohemmed, S. Schliebs, S. Matsuda, and N. Kasabov, "Method for training a spiking neuron to associate input-output spike trains," in Engineering Applications of Neural Network. Springer, 2011, pp. 219-228.

[64] A. Mohemmed and N. Kasabov, "Incremental learning algorithm for spatiotemporal spike pattern classification," in International Joint Conference on Neural Networks, 2012, pp. 1-6.

[65] A. Mohemmed, S. Schliebs, S. Matsuda, and N. Kasabov, "Training spiking neural networks to associate spatio-temporal input-output spike patterns," Neurocomputing, vol. 107, pp. 3-10, 2013.

[66] A. Mohemmed, S. Matsuda, S. Schliebs, K. Dhoble, and N. Kasabov, "Optimization of spiking neural networks with dynamic synapses for spike sequence generation using pso," in International Joint Conference on Neural Networks, 2011, pp. 29692974.

[67] Q. Yu, H. Tang, and K. C. Tan, "A new learning rule for classification of spatiotemporal spike patterns," in International Joint Conference on Neural Networks, 2014, pp. 3853-3858.

[68] Q. Yu, R. Yan, H. Tang, K. C. Tan, and H. Li, "A spiking neural network system for robust sequence recognition," IEEE Transactions on Neural Networks and Learning Systems, vol. 27, no. 3, pp. 621-635, 2016. 
[69] A. Mohemmed, S. Matsuda, S. Schliebs, K. Dhoble, and N. Kasabov, "Optimization of spiking neural networks with dynamic synapses for spike sequence generation using pso," in International Joint Conference on Neural Networks, 2011, pp. 29692974.

[70] S. Schliebs, M. Defoin-Platel, S. Worner, and N. Kasabov, "Integrated feature and parameter optimization for an evolving spiking neural network: Exploring heterogeneous probabilistic models," Neural Networks, vol. 22, no. 5, pp. 623-632, 2009.

[71] A. Delorme and S. J. Thorpe, "Face identification using one spike per neuron: resistance to image degradation," Neural Networks, vol. 14, no. 6, pp. 795-803, 2001.

[72] S. Schliebs and N. Kasabov, "Evolving spiking neural networka survey," Evolving Systems, vol. 4, no. 2, pp. 87-98, 2013.

[73] N. Kasabov, V. Feigin, Z.-G. Hou, Y. Chen, L. Liang, R. Krishnamurthi, M. Othman, and P. Parmar, "Evolving spiking neural networks for personalised modelling, classification and prediction of spatio-temporal patterns with a case study on stroke," Neurocomputing, vol. 134, pp. 269-279, 2014.

[74] M. Othman, N. Kasabov, E. Tu, V. Feigin, R. Krishnamurthi, Z. Hou, Y. Chen, and J. Hu, "Improved predictive personalized modelling with the use of spiking neural network system and a case study on stroke occurrences data," in International Joint Conference on Neural Networks, 2014, pp. 3197-3204.

[75] E. Capecci, N. Kasabov, and G. Y. Wang, "Analysis of connectivity in neucube spiking neural network models trained on eeg data for the understanding of functional changes in the brain: A case study on opiate dependence treatment," Neural Networks, vol. 68, pp. 62-77, 2015.

[76] E. Capecci, J. I. Espinosa-Ramos, N. Mammone, N. Kasabov, J. Duun-Henriksen, T. W. Kjaer, M. Campolo, F. L. Foresta, and F. C. Morabito, "Modelling absence 
epilepsy seizure data in the neucube evolving spiking neural network architecture," in International Joint Conference on Neural Networks, 2015, pp. 1-8.

[77] E. Capecci, F. C. Morabito, M. Campolo, N. Mammone, D. Labate, and N. Kasabov, "A feasibility study of using the neucube spiking neural network architecture for modelling alzheimers disease EEG data," in Advances in Neural Networks: Computational and Theoretical Issues. Springer, 2015.

[78] T. Gao and N. Kasabov, "Adaptive cow movement detection using evolving spiking neural network models," Evolving Systems, pp. 1-9, 2016.

[79] G. Sun, T. Chen, Z. Wei, Y. Sun, H. Zang, and S. Chen, "A carbon price forecasting model based on variational mode decomposition and spiking neural networks," Energies, vol. 9, no. 1, p. 54, 2016.

[80] S. Kulkarni, S. P. Simon, and K. Sundareswaran, "A spiking neural network (snn) forecast engine for short-term electrical load forecasting," Applied Soft Computing, vol. 13, no. 8, pp. 3628-3635, 2013.

[81] J. Wang, A. Belatreche, L. Maguire, and T. M. McGinnity, "An online supervised learning method for spiking neural networks with adaptive structure," Neurocomputing, vol. 144, pp. 526-536, 2014.

[82] S. Dora, K. Subramanian, S. Suresh, and N. Sundararajan, "Development of a self-regulating evolving spiking neural network for classification problem," Neurocomputing, vol. 171, pp. 1216-1229, 2016.

[83] Q. X. Wu, T. M. McGinnity, L. P. Maguire, B. Glackin, and A. Belatreche, "Learning under weight constraints in networks of temporal encoding spiking neurons," Neurocomputing, vol. 69, no. 16, pp. 1912-1922, 2006.

[84] S. S. Haykin, Neural networks and learning machines. Pearson Education Upper Saddle River, 2009.

[85] S. Suresh, S. Omkar, V. Mani, and T. G. Prakash, "Lift coefficient prediction at high angle of attack using recurrent neural network," Aerospace Science and Technology, vol. 7, no. 8, pp. 595-602, 2003. 
[86] G. Perea, M. Navarrete, and A. Araque, "Tripartite synapse: astrocytes process and control synaptic information," Trends in neurosciences, vol. 32, no. 8, pp. 421-431, 2009.

[87] E. A. Bushong, M. E. Martone, Y. Z. Jones, and M. H. Ellisman, "Protoplasmic astrocytes in CA1 stratum radiatum occupy separate anatomical domains," The Journal of neuroscience, vol. 22, no. 1, pp. 183-192, 2002.

[88] M. M. Halassa, T. Fellin, H. Takano, J. Dong, and P. G. Haydon, "Synaptic islands defined by the territory of a single astrocyte," The Journal of neuroscience, vol. 27, no. 24, pp. 6473-6477, 2007.

[89] A. Araque, V. Parpura, R. P. Sanzgiri, and P. G. Haydon, "Tripartite synapse: glia, the unacknowledged partner," Trends in neurosciences, vol. 22, no. 5, pp. 208-215, 1999.

[90] A. Volterra, P. J. Magistretti, and P. G. Haydon, The tripartite synapse: glia in synaptic transmission. Oxford University Press, 2002.

[91] T. Kohonen, Self-Organizing Maps. Springer, 2001.

[92] S. Suresh, K. Dong, and H. Kim, "A sequential learning algorithm for self-adaptive resource allocation network classifier," Neurocomputing, vol. 73, no. 16, pp. 30123019, 2010.

[93] G. Babu and S. Suresh, "Sequential projection-based metacognitive learning in a radial basis function network for classification problems," IEEE Transactions on Neural Networks and Learning Systems, vol. 24, no. 2, pp. 194-206, 2013.

[94] P. O'Connor, D. Neil, S.-C. Liu, T. Delbruck, and M. Pfeiffer, "Real-time classification and sensor fusion with a spiking deep belief network," Frontiers in Neuroscience, vol. 7, no. 178, 2013.

[95] S. B. Furber, D. R. Lester, L. A. Plana, J. D. Garside, E. Painkras, S. Temple, and A. D. Brown, "Overview of the spinnaker system architecture," IEEE Transactions on Computers, vol. 62, no. 12, pp. 2454-2467, 2013. 
[96] J. Schemmel, D. Bruderle, A. Grubl, M. Hock, K. Meier, and S. Millner, "A waferscale neuromorphic hardware system for large-scale neural modeling," in IEEE international symposium on circuits and systems, 2010, pp. 1947-1950.

[97] B. V. Benjamin, P. Gao, E. McQuinn, S. Choudhary, A. R. Chandrasekaran, J.-M. Bussat, R. Alvarez-Icaza, J. V. Arthur, P. A. Merolla, and K. Boahen, "Neurogrid: A mixed-analog-digital multichip system for large-scale neural simulations," Proceedings of the IEEE, vol. 102, no. 5, pp. 699-716, 2014.

[98] P. A. Merolla, J. V. Arthur, R. Alvarez-Icaza, A. S. Cassidy, J. Sawada, F. Akopyan, B. L. Jackson, N. Imam, C. Guo, Y. Nakamura et al., "A million spiking-neuron integrated circuit with a scalable communication network and interface," Science, vol. 345, no. 6197, pp. 668-673, 2014.

[99] D. Neil and S.-C. Liu, "Minitaur, an event-driven FPGA-based spiking network accelerator," Very Large Scale Integration (VLSI) Systems, IEEE Transactions on, vol. 22, no. 12, pp. 2621-2628, 2014.

[100] T. Pfeil, A. Grübl, S. Jeltsch, E. Müller, P. Müller, M. A. Petrovici, M. Schmuker, D. Brüderle, J. Schemmel, and K. Meier, "Six networks on a universal neuromorphic computing substrate," arXiv preprint arXiv:1210.7083, 2012.

[101] S.-C. Liu, T. Delbruck, G. Indiveri, A. Whatley, and R. Douglas, Event-Based Neuromorphic Systems. John Wiley \& Sons, 2015.

[102] S. W. Moore, P. J. Fox, S. J. Marsh, A. T. Markettos, and A. Mujumdar, "Bluehivea field-programable custom computing machine for extreme-scale real-time neural network simulation," in IEEE 20th Annual International Symposium on FieldProgrammable Custom Computing Machines (FCCM), 2012, pp. 133-140.

[103] S. B. Furber, F. Galluppi, S. Temple, and L. APlana, "The spinnaker project," Proceedings of the IEEE, vol. 102, no. 5, pp. 652-665, 2014.

[104] M. J. Pelgrom, A. C. Duinmaijer, and A. P. Welbers, "Matching properties of MOS transistors," IEEE Journal of solid-state circuits, vol. 24, no. 5, pp. 1433-1439, 1989. 
[105] A. Pavasović, A. G. Andreou, and C. R. Westgate, "Characterization of subthreshold mos mismatch in transistors for vlsi systems," Journal of VLSI signal processing systems for signal, image and video technology, vol. 8, no. 1, pp. 75-85, 1994.

[106] J. M. Brader, W. Senn, and S. Fusi, "Learning real-world stimuli in a neural network with spike-driven synaptic dynamics," Neural computation, vol. 19, no. 11, pp. 2881-2912, 2007.

[107] G. E. Hinton and R. R. Salakhutdinov, "Reducing the dimensionality of data with neural networks," Science, vol. 313, no. 5786, pp. 504-507, 2006.

[108] M. Schmuker, T. Pfeil, and M. P. Nawrot, "A neuromorphic network for generic multivariate data classification," Proceedings of the National Academy of Sciences, vol. 111, no. 6, pp. 2081-2086, 2014.

[109] N. Qiao, H. Mostafa, F. Corradi, M. Osswald, F. Stefanini, D. Sumislawska, and G. Indiveri, "A reconfigurable on-line learning spiking neuromorphic processor comprising 256 neurons and 128k synapses," Frontiers in neuroscience, vol. 9, no. 141, 2015.

[110] E. Neftci, S. Das, B. Pedroni, K. Kreutz-Delgado, and G. Cauwenberghs, "Eventdriven contrastive divergence for spiking neuromorphic systems," Frontiers in Neuroscience, vol. 7, no. 272, 2014.

[111] E. Stromatias, D. Neil, M. Pfeiffer, F. Galluppi, S. B. Furber, and S.-C. Liu, "Robustness of spiking deep belief networks to noise and reduced bit precision of neuroinspired hardware platforms," Frontiers in neuroscience, vol. 9, no. 222, 2015.

[112] A. J. Siegert, "On the first passage time probability problem," Physical Review, vol. 81 , no. 4 , p. $617,1951$.

[113] S. Hussain, R. Gopalakrishnan, A. Basu, and S.-C. Liu, "Morphological learning: Increased memory capacity of neuromorphic systems with binary synapses exploiting AER based reconfiguration," in International Joint Conference on Neural Networks, 2013, pp. 1-7. 
[114] S. Roy, P. P. San, S. Hussain, L. W. Wei, and A. Basu, "Learning spike time codes through morphological learning with binary synapses," IEEE Transactions on Neural Networks and Learning Systems, vol. 27, no. 7, pp. 1572-1577, 2015.

[115] S. Hussain, S.-C. Liu, and A. Basu, "Hardware-amenable structural learning for spike-based pattern classification using a simple model of active dendrites," Neural Computation, vol. 27, no. 4, pp. 845-897, 2015. 